\title{
Representations of Tame Quivers and Affine Canonical Bases
}

\author{
Dedicated to Claus Michael Ringel on the occasion of his 60th birthday
}

by

Zongzhu Lin, Jie XIAO and Guanglian Zhang

\begin{abstract}
There are several different ways to construct affine canonical bases, in addition to approaches by Lusztig and Kashiwara. In this paper we present a different approach to canonical bases via Hall algebras and representations of tame quivers over finite fields. The main idea is to tensor together integral bases constructed for cyclic quivers and Kronecker quivers with those from the preinjective and preprojective parts of tame quiver representations. Several different bases: a PBW type basis, a monomial basis, and a barinvariant basis are constructed and their relations to the canonical basis are discussed. The result also answers a question by Nakajima.
\end{abstract}

2010 Mathematics Subject Classification: Primary 17B37; Secondary 16G70.

Keywords: affine canonical bases, tame quivers, perverse sheaves.

\section{$\S 0$. Introduction}

\section{$\S 0.1$}

Let $U^{+}$be the positive part of the quantized enveloping algebra (over $\mathbb{C}(v)$ ) of a Kac-Moody Lie algebra $\mathfrak{g}$ associated to a Cartan datum $[\mathrm{Ka}]$. The construction of the canonical basis consists of a $\mathbb{Z}\left[v^{-1}\right]$-lattice $\mathcal{L}$ in Lusztig's integral $\mathcal{Z}=\mathbb{Z}\left[v, v^{-1}\right]$ form $U_{\mathcal{Z}}$ with a $\mathbb{Z}\left[v^{-1}\right]$-basis $B$ such that the restriction of the quotient map

Communicated by H. Nakajima. Received January 9, 2009. Revised August 8, 2010.

Z. Lin: Department of Mathematics, Kansas State University, Manhattan, KS 66506, U.S.A.; e-mail: zlin@math.ksu.edu

J. Xiao: Department of Mathematical Sciences, Tsinghua University, Beijing 10084, P.R. China; e-mail: jxiao@math.tsinghua.edu.cn

G. Zhang (corresponding author): Department of Mathematics, Shanghai Jiaotong University, Shanghai 200240, P.R. China;

e-mail: g.l.zhang@sjtu.edu.cn 
$\pi: \mathcal{L} \rightarrow \mathcal{L} / v^{-1} \mathcal{L}$ to $\mathcal{L} \cap \overline{\mathcal{L}}$ is an isomorphism of $\mathbb{Z}$-modules. The elements in $\mathbf{B}=\pi^{-1}(\pi(B)) \cap(\mathcal{L} \cap \overline{\mathcal{L}})$ are bar-invariant and form a $\mathcal{Z}$-basis of $U_{\mathcal{Z}}^{+}$. The set $\mathbf{B}$ is called the canonical basis. If the Cartan datum is of finite type, Lusztig used PBW bases for $B$, which are associated with reduced expressions of the longest element of the Weyl group, and the image $\pi(B)$ is independent of the reduced expressions $[\mathrm{L} 6, \mathrm{~L} 7, \mathrm{DL}]$.

If the Cartan datum is of finite or tame type, various approaches are taken to construct the integral basis $B$ for $U_{\mathcal{Z}}^{+}$. Lusztig's geometric approach, which uses certain simple perverse sheaves, gives $\mathbf{B}$ directly and works for general symmetric Cartan matrices [L1-L5]. Kashiwara's approach, which uses the Kashiwara operators, works for more general Kac-Moody Lie algebras. Kashiwara called the $\mathbb{Z}$-basis $\pi(B)$ the crystal basis and the $\mathcal{Z}$-basis $\mathbf{B}$ the global crystal basis $[\mathrm{K} 1, \mathrm{~K} 2, \mathrm{GL}]$. In the affine cases, Beck, Chari, and Pressley [BCP] constructed an integral basis for untwisted affine type. Then Beck and Nakajima [BN] improved and extended the results to all twisted affine types by using the Drinfeld realization $[\mathrm{Dr}]$ of affine quantum groups. The main goal of this paper is to provide another approach to constructing an integral basis and to discuss its relation to the canonical basis by using properties of representations of affine quivers. This approach is motivated by Lusztig's approach for finite type quiver cases, using Ringel's characterization of $U^{+}$as the generic Hall algebra of a finite type quiver.

\section{$\S 0.2$}

For a finite type root system, using Ringel's characterization of $U^{+}$as the generic Hall algebra of a Dynkin quiver, a PBW type basis for $U^{+}$can be realized as the set of isomorphism classes of representations of the quiver over finite fields. It uses an order compatible with the orbit closure inclusion relation in the representation varieties. In the affine cases, $U^{+}$is isomorphic to the (generic) composition algebra, which is a subalgebra of the Hall algebra. Not all isomorphism classes of representations of the affine Dynkin quiver are in $U^{+}$. For cyclic quivers, there are several approaches using quiver representations to construct the canonical basis. They include the work of Lusztig [L1, L2, L3, L5] and Varagnolo-Vaserot [VV, S] in terms of geometry and simple perverse sheaves, and [DDX] which uses computations in terms of quiver representations over finite fields. It should be mentioned that Lusztig's construction of the affine canonical basis in [L3] uses the McKay correspondence between certain affine quivers and finite subgroups of $\mathrm{SL}_{2}(\mathbb{C})$ and has the flavor of the Hall algebra approach. Lusztig's construction is also extended 
to all simply laced affine quivers using representations of the quiver over any algebraically closed field without reference to McKay correspondence [Lin, LL].

The main feature of this paper is to work for all tame quivers using computations in the Hall algebra. In this paper, an integral PBW type basis is constructed by tensoring bases from three subalgebras of the Hall algebra corresponding to the subcategories of preinjective, regular, and preprojective representations of the tame quiver. Not all regular modules are in the composition algebra. The regular part is further decomposed into the non-homogeneous part (corresponding to several cyclic quivers) and the homogeneous part. For the non-homogeneous regular part, bases of the composition algebra for cyclic quivers constructed in [DDX] can be used by identifying each non-homogeneous tube with the category of nilpotent representations of a cyclic quiver. By embedding the representation category of the Kronecker quiver into the representation category of the affine quiver, the image of the regular part of the Kronecker quiver together with those from non-homogeneous tubes would complete the PBW basis of the regular part.

The idea of using the embedding of representations of the Kronecker quiver appeared in [FMV] which motivated the present work. For the Kronecker quiver, a certain integral basis with the aforementioned triangular decomposition is constructed by Chen [C] following the work of Zhang [Z2]. In [Z1], Zhang studied the triangular decomposition structure of the composition algebra of affine type. In his preprint $[\mathrm{H}]$, Hubery refined the work of [Z1] and constructed a PBW type basis for the composition algebra using the triangular decomposition. The approach in this paper uses the idea of triangular decomposition of [Z1, Z2] and is similar to that in $[\mathrm{H}]$ when constructing a PBW type basis for the non-homogeneous regular part.

To relate this integral PBW basis to the canonical basis, one of the main passages is a monomial basis, which plays a significant role in Lusztig's construction in terms of perverse sheaves. The monomial basis elements are the direct images of the constant sheaves over Springer type resolutions of certain orbit closures. We will construct a monomial basis in terms of Hall algebras.

\section{$\S 0.3$}

For a finite type root system, Lusztig [L1] constructed the canonical basis in three different ways. The first is to construct an integral PBW basis $B$ by means of a braid group action on simple Chevalley generators as in [DL] using a reduced expression of the longest Weyl group element $w_{0}$. This is possible in a finite type root system since every root is conjugate to a simple root. The second approach is the aformentioned Hall approach where the integral basis elements are the iso- 
morphism classes of representations of the Dynkin graph with a fixed orientation. The third approach is to characterize the integral basis as certain semisimple complexes using the characterization of $U_{\mathcal{Z}}^{+}$as the (graded) Grothendieck group of a certain category of semisimple complexes in the equivariant derived category of constructible sheaves on the representation varieties of the Dynkin quiver.

This paper follows the idea of Lusztig's second approach together with the idea of using generic extension and the closure inclusion relation of orbits. One important property used in this paper is that Hall polynomials exist for regular representations of affine quivers (cf. [R2] and [Z2]). Using this property and direct computations on representations of affine quivers over finite fields, one can transfer these computations to the generic composition algebra, which has been identified with $U^{+}$. One has to be careful to keep track of the coefficients of $q$ as $q$ varies in order to derive a formula for $U^{+}$via Ringel's genericalization process (cf. §1.4).

\section{$\S 0.4$}

For an infinite type root system, the braid group action does not provide enough root vectors to construct a PBW type basis. For affine types, a PBW type basis was constructed in $[\mathrm{BCP}]$ and $[\mathrm{BN}]$. There, real root vectors were constructed by braid group actions on the Chevalley generators just as in the first approach for finite type case and imaginary root vectors were constructed by using Schur functions on Heisenberg generators. Then they used this PBW basis with the almost orthonormal property to obtain a global crystal basis (canonical basis). The approach of this paper is different from those of $[\mathrm{BCP}]$ and $[\mathrm{BN}]$ in that the regular simple modules corresponding to the real root vectors are in the regular part of the triangular decomposition mentioned in $\S 0.2$. Thus real root vectors can appear in all three parts of the triangular decomposition.

\section{$\S 0.5$}

We now explain the organization of this paper. In Section 1 we recall the definition of Hall algebras of quivers by Ringel and by Lusztig respectively, and establish an explicit relation between these two constructions. In particular, computations in one form can be translated to the other form. Representations of quivers in this section are over finite fields. Section 2 recalls some basic geometric properties of orbit varieties and extension varieties for representations of quivers over algebraically closed fields. Section 3 deals with cyclic quivers and is largely taken from 
[DDX], in which some integral bases and the canonical basis are constructed in terms of nilpotent representations of the cyclic quivers. The basis presented in this section is part of the basis needed in Section 6. In Section 4, we discuss the Hall subalgebra generated by preinjective modules and the Hall subalgebra generated by preprojective modules. In this case, we can construct a monomial basis in addition to an obvious PBW type basis in terms of isomorphism classes of modules. In Section 5 we recall, from [Z2] and [C], the construction of an integral PBW basis of $A_{1}^{(1)}$ type by using representations of the Kronecker quiver. In Section 6 , we show that the subalgebras corresponding to the preprojective part, preinjective part, non-homogeneous tubes, and the regular part of the Kronecker quiver can be tensored together to get a basis using the representation-directed property of the Auslander-Reiten quiver of a tame quiver. This gives rise to an integral basis of $U^{+}$over $\mathbb{Q}\left[v, v^{-1}\right]$. In Section 7 , we find a monomial basis which has a unipotent triangular relation with the integral PBW type basis constructed in Section 6 . Finally, a bar-invariant basis $\left\{\mathcal{E}^{\mathbf{c}} \mid \mathbf{c} \in \mathcal{M}\right\}$ of $U^{+}$can be constructed following Lusztig's argument. In Section 8, by a detailed calculation of the inner product on the PBW basis in the orthogonalization process using the properties of Schur functions, we can answer Nakajima's question in $[\mathrm{N}]$ affirmatively, by showing that the basis $\left\{\mathcal{E}^{\prime \mathbf{c}} \mid \mathbf{c} \in \mathcal{M}\right\}$, which is a modified form of the basis $\left\{\mathcal{E}^{\mathbf{c}} \mid \mathbf{c} \in \mathcal{M}\right\}$, equals the canonical basis in [L2]. The strong representation-directed property [DR] of the module category of the Kronecker quiver enables us in Section 9 to arrange the positive roots in a special order to give a direct computation of the canonical basis for the Kronecker quiver.

In a preprint $[\mathrm{Li}], \mathrm{Y}$. Li gives a geometric characterization of the monomial basis $\left\{m_{\mathbf{c}} \mid \mathbf{c} \in \mathcal{M}\right\}$ constructed by us in Section 8, and proves that the transition matrix between $\left\{m_{\mathbf{c}} \mid \mathbf{c} \in \mathcal{M}\right\}$ and the canonical basis is triangular with diagonal entries equal to 1 and entries above the diagonal in $\mathbb{Z}_{\geq 0}\left[v, v^{-1}\right]$.

\section{§1. Ringel-Hall algebras}

\section{$\S 1.1$}

A quiver $Q=(I, H, s, t)$ consists of a vertex set $I$, an arrow set $H$, and two maps $s, t: H \rightarrow I$ such that an arrow $\rho \in H$ starts at $s(\rho)$ and terminates at $t(\rho)$.

Throughout the paper, $\mathbb{F}_{q}$ denotes a finite field with $q$ elements, $Q=(I, H, s, t)$ is a fixed connected quiver without loops, and $\Lambda=\mathbb{F}_{q} Q$ is the path algebra of $Q$ over $\mathbb{F}_{q}$. By $\bmod \Lambda$ we denote the category of all finite-dimensional nilpotent left $\Lambda$-modules. We shall identify $\Lambda$-modules with representations of $Q$. 


\section{$\S 1.2$. Ringel-Hall algebra}

Given three modules $L, M, N$ in $\bmod \Lambda$, let $g_{M N}^{L}$ denote the number of $\Lambda$-submodules $W$ of $L$ such that $W \simeq N$ and $L / W \simeq M$ in $\bmod \Lambda$. More generally, for $M_{1}, \ldots, M_{t}, L \in \bmod \Lambda$, let $g_{M_{1} \cdots M_{t}}^{L}$ denote the number of filtrations $0=$ $L_{t} \subseteq L_{t-1} \subseteq \cdots \subseteq L_{1} \subseteq L_{0}=L$ of $\Lambda$-submodules such that $L_{i-1} / L_{i} \simeq M_{i}$ for $i=1, \ldots, t$. Let $v_{q}=\sqrt{q} \in \mathbb{C}$ and $\mathcal{P}$ be the set of isomorphism classes of finite-dimensional nilpotent $\Lambda$-modules. The Ringel-Hall algebra $\mathcal{H}(\Lambda)$ of $\Lambda$ is by definition the $\mathbb{Q}\left(v_{q}\right)$-vector space with basis $\left\{u_{[M]} \mid[M] \in \mathcal{P}\right\}$ whose multiplication is given by

$$
u_{[M]} u_{[N]}=\sum_{[L] \in \mathcal{P}} g_{M N}^{L} u_{[L]} .
$$

Note that $g_{M N}^{L}$ depends only on the isomorphism classes of $M, N$ and $L$. For a fixed pair of isomorphism classes $[M]$ and $[N]$, there are only finitely many isomorphism classes $[L]$ such that $g_{M N}^{L} \neq 0$. It is clear that $\mathcal{H}(\Lambda)$ is an associative $\mathbb{Q}\left(v_{q}\right)$-algebra with unit $u_{[0]}$, where 0 denotes the zero module.

The set of isomorphism classes of nilpotent simple $\Lambda$-modules is $\left\{S_{i} \mid i \in I\right\}$, where $S_{i}$ is one-dimensional at $i$ and zero elsewhere. Then the Grothendieck group $G(\Lambda)$ of $\bmod \Lambda$ is the free Abelian group $\mathbb{Z} I$. For each nilpotent $\Lambda$-module $M$, the dimension vector $\underline{\operatorname{dim}} M=\sum_{i \in I}\left(\operatorname{dim} M_{i}\right) i \in \mathbb{N} I$ is an element of $G(\Lambda)$. The Ringel-Hall algebra $\mathcal{H}(\Lambda)$ is graded by $\mathbb{N} I$, more precisely, by dimension vectors of modules.

The Euler form $\langle-,-\rangle$ on $G(\Lambda)=\mathbb{Z} I$ is defined by

$$
\langle\alpha, \beta\rangle=\sum_{i \in I} a_{i} b_{i}-\sum_{\rho \in H} a_{s(\rho)} b_{t(\rho)}
$$

for $\alpha=\sum_{i \in I} a_{i} i$ and $\beta=\sum_{i \in I} b_{i} i$ in $\mathbb{Z} I$. For any nilpotent $\Lambda$-modules $M$ and $N$,

$$
\langle\underline{\operatorname{dim}} M, \underline{\operatorname{dim}} N\rangle=\operatorname{dim}_{\mathbb{F}_{q}} \operatorname{Hom}_{\Lambda}(M, N)-\operatorname{dim}_{\mathbb{F}_{q}} \operatorname{Ext}_{\Lambda}(M, N) .
$$

The symmetric Euler form is defined as

$$
(\alpha, \beta)=\langle\alpha, \beta\rangle+\langle\beta, \alpha\rangle \quad \text { for } \alpha, \beta \in \mathbb{Z} I .
$$

This gives rise to a symmetric generalized Cartan matrix $C=\left(a_{i j}\right)_{i, j \in I}$ with $a_{i j}=(i, j)$. It is easy to see that $C$ is independent of the field $\mathbb{F}_{q}$ and the orientation of $Q$.

The twisted Ringel-Hall algebra $\mathcal{H}^{*}(\Lambda)$ is defined by setting $\mathcal{H}^{*}(\Lambda)=\mathcal{H}(\Lambda)$ as $\mathbb{Q}\left(v_{q}\right)$-vector space, but multiplication is defined by

$$
u_{[M]} * u_{[N]}=v_{q}^{\langle\underline{\operatorname{dim}} M, \underline{\operatorname{dim}} N\rangle} u_{[M]} u_{[N]} .
$$


Following [R3], for any $\Lambda$-module $M$, we denote $\langle M\rangle=v_{q}^{-\operatorname{dim} M+\operatorname{dim} \operatorname{End}_{\Lambda}(M)} u_{[M]}$. Note that $\{\langle M\rangle \mid M \in \mathcal{P}\}$ a $\mathbb{Q}\left(v_{q}\right)$-basis of $\mathcal{H}^{*}(\Lambda)$.

The $\mathbb{Q}\left(v_{q}\right)$-algebras $\mathcal{H}^{*}(\Lambda)$ and $\mathcal{H}(\Lambda)$ depend on $q$. We will use $\mathcal{H}_{q}^{*}(\Lambda)$ and $\mathcal{H}_{q}(\Lambda)$ to indicate the dependence on $q$ when such a need arises.

\section{§1.3. A construction by Lusztig}

For any finite-dimensional $I$-graded $\mathbb{F}_{q}$-vector space $V=\sum_{i \in I} V_{i}$, let $\mathbb{E}_{V}$ be the subset of $\bigoplus_{\rho \in H} \operatorname{Hom}_{\mathbb{F}_{q}}\left(V_{s(\rho)}, V_{t(\rho)}\right)$ defining nilpotent representations of $Q$. Note that $\mathbb{E}_{V}=\bigoplus_{\rho \in H} \operatorname{Hom}_{\mathbb{F}_{q}}\left(V_{s(\rho)}, V_{t(\rho)}\right)$ when $Q$ has no oriented cycles. The group $G_{V}=\prod_{i \in I} \mathrm{GL}\left(V_{i}\right)$ acts naturally on $\mathbb{E}_{V}$ by

$$
(g, x) \mapsto g \bullet x=x^{\prime} \quad \text { where } \quad x_{\rho}^{\prime}=g_{t(\rho)} x_{\rho} g_{s(\rho)}^{-1} \quad \text { for all } \rho \in H .
$$

Let $\mathbb{C}_{G}\left(\mathbb{E}_{V}\right)$ be the space of $G_{V}$-invariant functions $\mathbb{E}_{V} \rightarrow \mathbb{C}$. For $\gamma \in \mathbb{N} I$, we fix an $I$-graded $\mathbb{F}_{q}$-vector space $V_{\gamma}$ with $\underline{\operatorname{dim}} V_{\gamma}=\gamma$. For example, $V_{\gamma}=\mathbb{F}_{q}^{\gamma}=\bigoplus_{i \in I} \mathbb{F}_{q}^{n_{i}}$ if $\gamma=\sum_{i \in I} n_{i} i$. We will simply denote $\mathbb{E}_{\gamma}=\mathbb{E}_{V_{\gamma}}$ and $G_{\gamma}=G_{V_{\gamma}}$. For $\alpha, \beta \in \mathbb{N} I$ and $\gamma=\alpha+\beta$, we consider the diagram

$$
\mathbb{E}_{\alpha} \times \mathbb{E}_{\beta} \stackrel{p_{1}}{\longleftarrow} \mathbb{E}^{\prime} \stackrel{p_{2}}{\longrightarrow} \mathbb{E}^{\prime \prime} \stackrel{p_{3}}{\longrightarrow} \mathbb{E}_{\gamma} .
$$

Here $\mathbb{E}^{\prime \prime}$ is the set of all pairs $(x, W)$ consisting of an $I$-graded subspace $W$ of $V_{\gamma}$ with $\underline{\operatorname{dim}} W=\beta$ and $x \in \mathbb{E}_{\gamma}$ such that $x_{\rho} W_{s(\rho)} \subseteq W_{t(\rho)}$ (i.e., $W$ is $x$-stable); $\mathbb{E}^{\prime}$ is the set of all quadruples $\left(x, W, R^{\prime}, R^{\prime \prime}\right)$ consisting of $(x, W) \in \mathbb{E}^{\prime \prime}$ and invertible graded linear maps $R^{\prime \prime}: \mathbb{F}_{q}^{\beta} \rightarrow W$ and $R^{\prime}: \mathbb{F}_{q}^{\alpha} \rightarrow V_{\gamma} / W$. The maps $p_{2}$ and $p_{3}$ are the obvious projections and $p_{1}\left(x, W, R^{\prime}, R^{\prime \prime}\right)=\left(x^{\prime}, x^{\prime \prime}\right)$, where $x_{\rho}^{\prime \prime}=\left.\left(R_{t(\rho)}^{\prime \prime}\right)^{-1} x_{\rho}\right|_{W_{s(\rho)}} R_{s(\rho)}^{\prime \prime}$ and $x_{\rho}^{\prime}=\left(R_{t(\rho)}^{\prime}\right)^{-1} \bar{x}_{\rho} R_{s(\rho)}^{\prime}$ for all $\rho \in H$. Here $\bar{x}_{\rho}:\left(V_{\gamma} / W\right)_{s(\rho)} \rightarrow\left(V_{\gamma} / W\right)_{t(\rho)}$ is the quotient map induced from $x_{\rho}$.

For any map $p: X \rightarrow Y$ of finite sets, $p^{*}: \mathbb{C}(Y) \rightarrow \mathbb{C}(X)$ is defined by $p^{*}(f)(x)=f(p(x))$ and $p_{!}: \mathbb{C}(X) \rightarrow \mathbb{C}(Y)$ is defined by $p_{!}(h)(y)=\sum_{x \in p^{-1}(y)} h(x)$ (integration along the fibers). Given $f \in \mathbb{C}_{G}\left(\mathbb{E}_{\alpha}\right)$ and $g \in \mathbb{C}_{G}\left(\mathbb{E}_{\beta}\right)$, there is a unique $h \in \mathbb{C}_{G}\left(\mathbb{E}^{\prime \prime}\right)$ such that $p_{2}^{*}(h)=p_{1}^{*}(f \times g)$. Then define

$$
f \circ g=\left(p_{3}\right) !(h) \in \mathbb{C}_{G}\left(\mathbb{E}_{\gamma}\right) .
$$

Note that the isomorphism classes of nilpotent $\Lambda$-modules of a fixed dimension vector $\alpha$ are in one-to-one correspondence with $G_{\alpha}$-orbits in $\mathbb{E}_{\alpha}$. For each $x \in \mathbb{E}_{\alpha}$, let $M_{x}$ be the $\Lambda$-module defined by $x, \mathcal{O}_{x}=G_{\alpha} \bullet x$ be the $G_{\alpha}$-orbit, and $\mathbf{1}_{\mathcal{O}_{x}}$ be the characteristic function of the finite set $\mathcal{O}_{x}$. Then we have

$$
\mathbf{1}_{\mathcal{O}_{x}} \circ \mathbf{1}_{\mathcal{O}_{y}}(z)=g_{M_{x}, M_{y}}^{M_{z}}
$$


for all $x \in \mathbb{E}_{\alpha}, y \in \mathbb{E}_{\beta}$, and $z \in \mathbb{E}_{\gamma}$. Therefore there is a natural isomorphism of $\mathbb{C}$-algebras

$$
\mathcal{H}_{q}(\Lambda) \otimes_{\mathbb{Q}\left(v_{q}\right)} \mathbb{C} \rightarrow\left(\bigoplus_{\alpha \in \mathbb{N} I} \mathbb{C}_{G}\left(\mathbb{E}_{\alpha}\right), \circ\right)
$$

sending $u_{\left[M_{x}\right]}$ to $\mathbf{1}_{\mathcal{O}_{x}}$.

Let

$$
\mathbf{m}(\alpha, \beta)=\sum_{i \in I} a_{i} b_{i}+\sum_{\rho \in H} a_{s(\rho)} b_{t(\rho)} .
$$

We again define multiplication in the $\mathbb{C}$-space $\mathbf{K}=\bigoplus_{\alpha \in \mathbb{N} I} \mathbb{C}_{G}\left(\mathbb{E}_{\alpha}\right)$ by

$$
f * g=v_{q}^{-\mathbf{m}(\alpha, \beta)} f \circ g
$$

for all $f \in \mathbb{C}_{G}\left(\mathbb{E}_{\alpha}\right)$ and $g \in \mathbb{C}_{G}\left(\mathbb{E}_{\beta}\right)$. Then $(\mathbf{K}, *)$ becomes an associative $\mathbb{C}$-algebra.

Conventions. (1) To simplify the notation, instead of writing $M_{x}$ for each $x \in \mathbb{E}_{\alpha}$, we will simply write $M \in \mathbb{E}_{\alpha}$ by using $M$ as both an element of $\mathbb{E}_{\alpha}$ and the corresponding $\Lambda$-module. Thus $\mathcal{O}_{M} \subseteq \mathbb{E}_{\alpha}$ is the corresponding $G_{\alpha}$-orbit.

(2) Although we are working over a finite field $\mathbb{F}_{q}$, we will regularly use $G_{V}$ and $\mathbb{E}_{V}$ for the algebraic group and the algebraic variety over $\overline{\mathbb{F}}_{q}$ with an obvious $\mathbb{F}_{q}$-structure and use the features of algebraic geometry without introducing extra notation, i.e., the set of $\mathbb{F}_{q}$-rational points and the algebraic variety are denoted by the same symbol. This should not cause any confusion and the meanings should be clear from the context. In particular, since stabilizer subgroups are connected algebraic groups, Lang's theorem ensures that two $\mathbb{F}_{q}$-rational points are in the same orbit under the algebraic group action if and only if they are in the same orbit under the finite group of $\mathbb{F}_{q}$-rational points.

For $M \in \mathbb{E}_{\alpha}$, let $\mathcal{O}_{M} \subset \mathbb{E}_{\alpha}$ be the $G_{\alpha}$-orbit of $M$. We take $\mathbf{1}_{[M]} \in \mathbb{C}_{G}\left(V_{\alpha}\right)$ to be the characteristic function of $\mathcal{O}_{M}$, and set $f_{[M]}=v_{q}^{-\operatorname{dim} \mathcal{O}_{M}} \mathbf{1}_{[M]}$. We consider the subalgebra $(\mathbf{L}, *)$ of $(\mathbf{K}, *)$ generated by $f_{[M]}$ over $\mathbb{Q}\left(v_{q}\right)$, for all $M \in \mathbb{E}_{\alpha}$ and all $\alpha \in \mathbb{N} I$. In fact $\mathbf{L}$ has a $\mathbb{Q}\left(v_{q}\right)$-basis $\left\{f_{[M]} \mid M \in \mathbb{E}_{\alpha}, \alpha \in \mathbb{N} I\right\}$.

Proposition 1.1. The linear map $\varphi:(\mathbf{L}, *) \rightarrow \mathcal{H}^{*}(\Lambda)$ defined by

$$
\varphi\left(f_{[M]}\right)=\langle M\rangle \quad \text { for all }[M] \in \mathcal{P}
$$

is an isomorphism of associative $\mathbb{Q}\left(v_{q}\right)$-algebras.

Proof. By definition, $\phi$ is a linear isomorphism. For $M \in \mathbb{E}_{\alpha}$, one first notes that $\operatorname{dim} \mathcal{O}_{M}=\operatorname{dim} G_{\alpha}-\operatorname{dim} \operatorname{End}_{\Lambda}(M)$ and $\operatorname{dim} G_{\alpha+\beta}-\operatorname{dim} G_{\alpha}-\operatorname{dim} G_{\beta}=$ 
$\langle\alpha, \beta\rangle+\mathbf{m}(\alpha, \beta)$. If $N \in \mathbb{E}_{\beta}$ and $L \in \mathbb{E}_{\alpha+\beta}$, then

$$
\begin{aligned}
\operatorname{dim} \operatorname{End}_{\Lambda}(M)+\operatorname{dim} \operatorname{End}_{\Lambda}(N)-\operatorname{dim} \operatorname{End}_{\Lambda}(L)+\langle\alpha, \beta\rangle \\
\quad=-\operatorname{dim} \mathcal{O}_{M}-\operatorname{dim} \mathcal{O}_{N}+\operatorname{dim} \mathcal{O}_{L}-\mathbf{m}(\alpha, \beta) .
\end{aligned}
$$

Now a direct computation of the structure constants for the bases $\left\{f_{[M]}\right\}$ and $\{\langle M\rangle\}$, respectively, will finish the proof.

\section{§1.4. Genericalization}

The free abelian group $G(\Lambda)=\mathbb{Z} I$ with the symmetric Euler form $(-,-)$ defined in $\S 1.2$ is a Cartan datum in the sense of Lusztig [L5]. Associated to $(\mathbb{Z} I,(-,-))$ is the Drinfeld-Jimbo quantized enveloping algebra $U=U^{-} \otimes U^{0} \otimes U^{+}$defined over $\mathbb{Q}(v)$, where $v$ is transcendental over $\mathbb{Q}$. It is generated by the Chevalley generators $E_{i}, F_{i}, K_{i}^{ \pm}(i \in I)$ with respect to the quantum Serre relations. Recall that $\mathcal{Z}=\mathbb{Z}\left[v, v^{-1}\right] \subseteq \mathbb{Q}(v)$. The Lusztig $\mathcal{Z}$-form $U_{\mathcal{Z}}^{+}$of $U^{+}$is the $\mathcal{Z}$-subalgebra in $U^{+}$generated by $E_{i}^{(m)}=E_{i}^{m} /[m] !(m \geq 0$ and $i \in I)$, where

$$
[n]=\frac{v^{n}-v^{-n}}{v-v^{-1}}, \quad[n] !=\prod_{r=1}^{n}[r], \quad\left[\begin{array}{l}
n \\
r
\end{array}\right]=\frac{[n] !}{[r] ![n-r] !} .
$$

For $v_{q}=\sqrt{q} \in \mathbb{C}$, let $\mathcal{Z}_{v_{q}}$ be the subring of $\mathbb{C}$ which is the image of $\mathcal{Z}$ under the map $\mathcal{Z} \rightarrow \mathbb{C}$ with $v \mapsto v_{q}$. Let $\mathcal{C}^{*}(\Lambda)_{\mathcal{Z}_{v_{q}}}$ be the $\mathcal{Z}_{v_{q}}$-subalgebra of $\mathcal{H}_{q}^{*}(\Lambda)$ generated by $u_{\left[S_{i}\right]}^{(* m)}=u_{\left[S_{i}\right]}^{* m} /[m]_{v_{q}} !(i \in I)$, where $[n]_{v_{q}} \in \mathcal{Z}_{v_{q}}$ is the image of $[n]$ in $\mathcal{Z}_{v_{q}}$.

It follows from the works of Ringel [R1], Green [G], and Sevenhant-Van den Bergh $[\mathrm{SV}]$ that $\mathcal{C}^{*}(\Lambda)_{\mathcal{Z}_{v_{q}}}$ is isomorphic to $U_{\mathcal{Z}}^{+} \otimes_{\mathcal{Z}} \mathcal{Z}_{v_{q}}$ by sending $u_{\left[S_{i}\right]}^{(* m)}$ to $E_{i}^{(m)}$.

We will denote $U_{\mathcal{Z}}^{+}$by $\mathcal{C}^{*}(\Lambda)_{\mathcal{Z}}$ and call it the integral generic composition algebra. In fact, following Ringel's argument, $\mathcal{Z}$ can be identified with the subring of $\prod_{q} \mathcal{Z}_{v_{q}}$ generated by $v^{ \pm 1}=\left(v_{q}^{ \pm 1}\right)$, and $\mathcal{C}^{*}(\Lambda)_{\mathcal{Z}}$ with the $\mathcal{Z}$-subalgebra of $\prod_{q} \mathcal{H}_{q}^{*}(\Lambda)$ generated by $\left(u_{\left[S_{i} \otimes \mathbb{F}_{q}\right]}^{(* m)}\right), m \geq 1$. Here the product is taken over all $q$ (although infinitely many will be enough).

In this paper, computations in $\prod_{q} \mathcal{H}_{q}^{*}(\Lambda)$ will be performed in each component. When an expression in each component is written as an element of $\mathbb{Z}\left[v_{q}, v_{q}^{-1}\right]$ with coefficients in $\mathbb{Z}$ independent of the choice of the field $\mathbb{F}_{q}$, we say that the expression is invariant (or generic) as $\mathbb{F}_{q}$ varies. In this case replacing $v_{q}$ by $v$ we will get a formula in $\prod_{q} \mathcal{H}_{q}^{*}(\Lambda)$. We will not repeat this replacement each time and simply write $v=v_{q}$ and call it generic in this expression. 
The bar involution $\overline{()}: U^{+} \rightarrow U^{+}$is a $\mathbb{Q}$-algebra automorphism defined by $\bar{v}=v^{-1}, \overline{E_{i}}=E_{i}$ and $\overline{E_{i}^{(m)}}=E_{i}^{(m)}$. Then $\overline{U_{\mathcal{Z}}^{+}}=U_{\mathcal{Z}}^{+}$. It should be pointed out that the bar operation is not defined when $v$ is specialized to $v_{q}$.

\section{$\S 1.5$}

In general, if we take a special value $v_{q}=\sqrt{q}$ for the finite field $\mathbb{F}_{q}$, it is easy to obtain

Lemma 1.2. Any monomial $\mathfrak{m}$ in $u_{S_{i}}^{(m)}, i \in I, m \in \mathbb{N}$, can be written as $\mathfrak{m}=$ $\sum_{M \in \mathcal{P}} f_{M, q}\langle M\rangle$ in $\mathcal{H}_{q}^{*}(\Lambda)$ with $f_{M, q} \in \mathcal{Z}_{v_{q}}$ such that for each $M$, there is an integer $b$ such that $v_{q}^{b} f_{M, q} \in \mathbb{Z}\left[v_{q}\right]$ (the subring of algebraic integers) and $b$ is independent of $\mathbb{F}_{q}$.

\section{$\S 2$. The variety of representations}

In this section, we list some geometric properties of representations of quivers over the algebraically closed field $k=\overline{\mathbb{F}}_{q}$. Take $\Lambda=k Q$; all Hom and Ext are taken in $\Lambda$-mod.

\section{$\S 2.1$}

For $\alpha \in \mathbb{N} I$, the $I$-graded $k$-vector space $V=\bigoplus_{i \in I} k^{\alpha_{i}}$ defines the affine algebraic $k$-variety $\bigoplus_{\rho \in H} \operatorname{Hom}_{k}\left(k^{\alpha_{s(\rho)}}, k^{\alpha_{t(\rho)}}\right)$ containing the set $\mathbb{E}_{\alpha}$ of all nilpotent elements as a closed subvariety on which the algebraic group $G_{\alpha}=\prod_{i \in I} \mathrm{GL}_{\alpha_{i}}(k)$ acts as in $\S 1.3$. For any $x \in \mathbb{E}_{\alpha}$, let $M_{x}$ be the corresponding representation of $Q$ over $k$. We will follow the convention (1) of $\S 1.3$ by simply writing $M \in \mathbb{E}_{\alpha}$ for a module $M$. The following properties are well-known (see [CB]).

Lemma 2.1. For any $\alpha \in \mathbb{N} I$ and $M \in \mathbb{E}_{\alpha}$, we have:

(i) $\operatorname{dim} \mathbb{E}_{\alpha}-\operatorname{dim} \mathcal{O}_{M}=\operatorname{dim}$ End $M-(\alpha, \alpha) / 2=\operatorname{dim} \operatorname{Ext}^{1}(M, M)$.

(ii) $\mathcal{O}_{M}$ is open in $\mathbb{E}_{\alpha}$ if and only if $M$ has no self-extension.

(iii) There is at most one orbit $\mathcal{O}_{M}$ in $\mathbb{E}_{\alpha}$ such that $M$ has no self-extension.

(iv) If $0 \rightarrow M \rightarrow L \rightarrow N \rightarrow 0$ is a non-split exact sequence, then $\mathcal{O}_{M \oplus N} \subseteq$ $\overline{\mathcal{O}}_{L} \backslash \mathcal{O}_{L}$.

(v) If $\mathcal{O}_{L}$ is an orbit in $\mathbb{E}_{\alpha}$ of maximal dimension and $L=M \oplus N$, then $\operatorname{Ext}^{1}(M, N)=0$. 
For subsets $\mathcal{A} \subset \mathbb{E}_{\alpha}$ and $\mathcal{B} \subset \mathbb{E}_{\beta}$, we define the extension set $\mathcal{A} \star \mathcal{B}$ of $\mathcal{A}$ by $\mathcal{B}$ to be

$$
\begin{aligned}
& \mathcal{A} \star \mathcal{B}=\left\{z \in \mathbb{E}_{\alpha+\beta} \mid\right. \text { there exists an exact sequence } \\
& \left.\qquad 0 \rightarrow M_{x} \rightarrow M_{z} \rightarrow M_{y} \rightarrow 0 \text { with } x \in \mathcal{B}, y \in \mathcal{A}\right\} .
\end{aligned}
$$

If $\mathcal{A}$ is a subvariety of $\mathbb{E}_{\alpha}$, then $\operatorname{codim} \mathcal{A}=\operatorname{dim} \mathbb{E}_{\alpha}-\operatorname{dim} \mathcal{A}$ is called the codimension of $\mathcal{A}$ in $\mathbb{E}_{\alpha}$. From $[\mathrm{Re}]$ we obtain

Lemma 2.2. Given any $\alpha, \beta \in \mathbb{N} I$, if $\mathcal{A} \subset \mathbb{E}_{\alpha}$ and $\mathcal{B} \subset \mathbb{E}_{\beta}$ are irreducible algebraic varieties and are stable under the actions of $G_{\alpha}$ and $G_{\beta}$ respectively, then $\mathcal{A} \star \mathcal{B}$ is irreducible and stable under the action of $G_{\alpha+\beta}$. Moreover,

$$
\operatorname{codim} \mathcal{A} \star \mathcal{B}=\operatorname{codim} \mathcal{A}+\operatorname{codim} \mathcal{B}-\langle\beta, \alpha\rangle+r
$$

where $0 \leq r \leq \min \left\{\operatorname{dim}_{k} \operatorname{Hom}\left(M_{y}, M_{x}\right) \mid y \in \mathcal{B}, x \in \mathcal{A}\right\}$.

\section{$\S 2.2$}

For any $\alpha, \beta \in \mathbb{N} I$, we consider the diagram of algebraic $k$-varieties

$$
\mathbb{E}_{\alpha} \times \mathbb{E}_{\beta} \stackrel{p_{1}}{\longleftarrow} \mathbb{E}^{\prime} \stackrel{p_{2}}{\longrightarrow} \mathbb{E}^{\prime \prime} \stackrel{p_{3}}{\longrightarrow} \mathbb{E}_{\alpha+\beta}
$$

defined in a similar way as in $\S 1.3$. It follows from the definition that $\mathcal{A} \star \mathcal{B}=$ $p_{3} p_{2}\left(p_{1}^{-1}(\mathcal{A} \times \mathcal{B})\right)$. Thus we have $\overline{\mathcal{A}} \star \overline{\mathcal{B}} \subseteq \overline{\mathcal{A} \star \mathcal{B}}$ since $p_{1}$ is a locally trivial fibration (see Lemma 2.3 below). For any $M \in \mathbb{E}_{\alpha}, N \in \mathbb{E}_{\beta}$, and $L \in \mathbb{E}_{\alpha+\beta}$ we define

$$
\mathbf{Z}=p_{2} p_{1}^{-1}\left(\mathcal{O}_{M} \times \mathcal{O}_{N}\right), \quad \mathbf{Z}_{L, M, N}=\mathbf{Z} \cap p_{3}^{-1}(L)
$$

The following properties can be found in [L1].

Lemma 2.3. For the diagram above and $M \in \mathbb{E}_{\alpha}, N \in \mathbb{E}_{\beta}$, and $L \in \mathbb{E}_{\alpha+\beta}$, we have the following properties:

(i) The map $p_{2}$ is a principal $G_{\alpha} \times G_{\beta}$ fibration.

(ii) The map $p_{1}$ is a locally trivial fibration with smooth connected fibers of dimension

$$
\sum_{i \in I} a_{i}^{2}+\sum_{i \in I} b_{i}^{2}+\mathbf{m}(\alpha, \beta)
$$

(iii) The map $p_{3}$ is proper.

(iv) The variety $\mathbf{Z}$ is smooth and irreducible of dimension

$$
\operatorname{dim} \mathbf{Z}=\operatorname{dim} \mathcal{O}_{M}+\operatorname{dim} \mathcal{O}_{N}+\mathbf{m}(\alpha, \beta) .
$$


(v) If $L$ is an extension of $M$ by $N$, then

$$
\operatorname{dim} \mathcal{O}_{L} \leq \operatorname{dim} \mathcal{O}_{M}+\operatorname{dim} \mathcal{O}_{N}+\mathbf{m}(\alpha, \beta) .
$$

(vi) If $\mathcal{O}_{L}$ is dense in $p_{3} \mathbf{Z}$, then

$$
\operatorname{dim} \mathcal{O}_{L}=\operatorname{dim} \mathcal{O}_{M}+\operatorname{dim} \mathcal{O}_{N}+\mathbf{m}(\alpha, \beta)-\operatorname{dim} \mathbf{Z}_{L, M, N} .
$$

(vii) Assume that $\operatorname{Ext}^{1}(M, N)=0$ and $\operatorname{Hom}(N, M)=0$. If $M^{\prime} \in \overline{\mathcal{O}}_{M}$ and $N^{\prime} \in$ $\overline{\mathcal{O}}_{N}$ with either $M^{\prime} \in \overline{\mathcal{O}}_{M} \backslash \mathcal{O}_{M}$ or $N^{\prime} \in \overline{\mathcal{O}}_{N} \backslash \mathcal{O}_{N}$, then $X \in \overline{\mathcal{O}}_{M \oplus N} \backslash \mathcal{O}_{M \oplus N}$ for all $X \in \overline{\mathcal{O}}_{M^{\prime}} \star \overline{\mathcal{O}}_{N^{\prime}}$. In particular, $\operatorname{dim} \mathcal{O}_{X}<\operatorname{dim} \mathcal{O}_{M \oplus N}$.

Lemma 2.4. Given any two representations $M$ and $N$ of $Q$ over $k$, if $\operatorname{Ext}^{1}(M, N)$ $=0$, then $\overline{\mathcal{O}}_{M} \star \overline{\mathcal{O}}_{N}=\overline{\mathcal{O}}_{M \oplus N}$, i.e., $\mathcal{O}_{M \oplus N}$ is open and dense in $\overline{\mathcal{O}}_{M} \star \overline{\mathcal{O}}_{N}$.

Proof. By definition, $\operatorname{Ext}^{1}(M, N)=0$ implies $\mathcal{O}_{M} \star \mathcal{O}_{N}=\mathcal{O}_{M \oplus N}$. Hence $\mathcal{O}_{M \oplus N}=$ $\mathcal{O}_{M} \star \mathcal{O}_{N} \subseteq \overline{\mathcal{O}}_{M} \star \overline{\mathcal{O}}_{N} \subseteq \overline{\mathcal{O}_{M} \star \mathcal{O}_{N}}=\overline{\mathcal{O}}_{M \oplus N}$. Therefore $\overline{\mathcal{O}}_{M} \star \overline{\mathcal{O}}_{N}=\overline{\mathcal{O}}_{M \oplus N}$, since any orbit (under any connected algebraic group action) is always open in its closure.

Lemma 2.5. Let $M, N, X \in \bmod \Lambda$. Then $\mathcal{O}_{X}$ is open in $\mathcal{O}_{M} \star \mathcal{O}_{N}$ if and only if $\mathcal{O}_{X}$ is open in $\overline{\mathcal{O}}_{M} \star \overline{\mathcal{O}}_{N}$. In that case for any $Y \in \overline{\mathcal{O}}_{M} \star \overline{\mathcal{O}}_{N}$ we have $\operatorname{dim} \mathcal{O}_{Y} \leq$ $\operatorname{dim} \mathcal{O}_{X}$.

Proof. This follows from $\mathcal{O}_{X} \subseteq \mathcal{O}_{M} \star \mathcal{O}_{N} \subseteq \overline{\mathcal{O}}_{M} \star \overline{\mathcal{O}}_{N} \subseteq \overline{\mathcal{O}_{M} \star \mathcal{O}_{N}}$ and Lemma 2.2.

To end this section, we recall the (geometric) partial order on $\mathcal{P}$ (the isomorphism classes) defined by Lusztig for any quiver: $[M] \preceq[N]$ if $\underline{\operatorname{dim}} M=\underline{\operatorname{dim}} N$ and $\mathcal{O}_{M} \subseteq \overline{\mathcal{O}}_{N}$. This order will play an important role in constructions of various bases.

\section{§3. The integral and canonical bases arising from a tube}

In this section we summarize some results from [DDX] on constructions of monomial bases and PBW bases for cyclic quivers. In [DDX] the canonical bases of $U_{q}\left(\hat{s l}{ }_{n}\right)$ and $U_{q}\left(\hat{g l} l_{n}\right)$ are constructed from these monomial bases by a linear algebra method from the category of finite-dimensional nilpotent representations of a cyclic quiver, i.e., from a tube.

\section{$\S 3.1$}

Let $\Delta=\Delta(n)$ be the cyclic quiver with vertex set $I=\mathbb{Z} / n \mathbb{Z}=\{1, \ldots, n\}$ and arrow set $H=\{i \rightarrow i+1 \mid i \in \mathbb{Z} / n \mathbb{Z}\}$. We consider the category $\mathcal{T}=\mathcal{T}(n)$ of 
finite-dimensional nilpotent representations of $\Delta(n)$ over $\mathbb{F}_{q}$. In view of the shape of its Auslander-Reiten quiver, $\mathcal{T}(n)$ is called a tube of rank $n$. The irreducible objects in $\mathcal{T}(n)$ are $\left\{S_{i} \mid i \in I\right\}$. Let $S_{i}[l]$ be the unique absolutely indecomposable object in $\mathcal{T}(n)$ with simple quotient $S_{i}$ and length $l$. Note that any indecomposable object in $\mathcal{T}(n)$ is isomorphic to an $S_{i}[l]$. Again in this section, we let $\mathcal{P}$ be the set of isomorphism classes of objects in $\mathcal{T}(n), \mathcal{H}$ the Ringel-Hall algebra of $\mathcal{T}(n)$, $\mathcal{H}^{*}$ the twisted Ringel-Hall algebra, and $\mathbf{L}$ the Lusztig form of the Hall algebra of $\mathcal{T}(n)$ (cf. §1.3). Because the Hall polynomials always exist in this case (see [R2]), we may regard the algebras $\mathcal{H}, \mathcal{H}^{*}$ and $\mathbf{L}$ in their generic form. So they are defined generically over $\mathbb{Q}(v)$ with $v$ being an indeterminate. By Proposition 1.1 , we may identify $\mathbf{L}$ with $\mathcal{H}^{*}$ via the morphism $\varphi$.

In this section, all properties we obtain are generic and independent of the base field $\mathbb{F}_{q}$, although the computations will be performed over $\mathbb{F}_{q}$ (for each $q$ ) following the remark in $\S 1.4$. We will omit the subscript $q$ for simplicity. Since the number $n$ is fixed, sometimes it is omitted too, e.g., $\mathcal{T}=\mathcal{T}(n)$.

\section{$\$ 3.2$}

Let $\lambda=\left(\lambda_{1} \geq \cdots \geq \lambda_{l}>0\right)$ be a partition of an integer $m$. We call $l$ and $m$, respectively, the length and size of the partition $\lambda$ and denote $|\lambda|=m$. Let $\Pi$ be the set of $n$-tuples $\pi=\left(\pi^{(1)}, \ldots, \pi^{(n)}\right)$ with each component $\pi^{(i)}=\left(\pi_{1}^{(i)} \geq \pi_{2}^{(i)} \geq \cdots\right)$ being a partition of an integer. Similarly, we write $|\pi|=\left|\pi^{(1)}\right|+\cdots+\left|\pi^{(n)}\right|$ and call it the size of $\pi$. For each $\pi \in \Pi$, we define an object in $\mathcal{T}$ by

$$
M(\pi)=\bigoplus_{\substack{i \in \Delta_{0} \\ j \geq 1}} S_{i}\left[\pi_{j}^{(i)}\right] .
$$

Every object in $\mathcal{T}$ is isomorphic to exactly one $M(\pi)$. This defines a bijection between $\Pi$ and $\mathcal{P}$. We will simply write $u_{\pi}$ for $u_{[M(\pi)]}$ in $\mathcal{H}$. The geometric partial order on $\mathcal{P}$ defines a partial order on $\Pi$ such that $\pi \preceq \pi^{\prime}$ if and only if $M(\pi) \preceq M\left(\pi^{\prime}\right)$.

An $n$-tuple $\pi=\left(\pi^{(1)}, \ldots, \pi^{(n)}\right)$ of partitions in $\Pi$ is called aperiodic (in the sense of Lusztig [L3]), or separated (in the sense of Ringel [R2]), if for each $l \geq 1$ there is some $i=i(l) \in I$ such that $\pi_{j}^{(i)} \neq l$ for all $j \geq 1$. By $\Pi^{a}$ we denote the set of aperiodic $n$-tuples of partitions. An object $M$ in $\mathcal{T}$ is called aperiodic if $M \simeq M(\pi)$ for some $\pi \in \Pi^{a}$. For any dimension vector $\alpha \in \mathbb{N}^{n}(=\mathbb{N} I)$, we let

$$
\Pi_{\alpha}=\{\pi \in \Pi \mid \underline{\operatorname{dim}} M(\pi)=\alpha\} \quad \text { and } \quad \Pi_{\alpha}^{a}=\Pi^{a} \cap \Pi_{\alpha} .
$$


For each $\alpha$ fixed, $\Pi_{\alpha}$ is finite. Thus $\mathbb{E}_{\alpha}$ has only finitely many $G_{\alpha}$-orbits. Therefore, for any two modules $M$ and $N$, there is a unique $L$ such that $\mathcal{O}_{L}$ has the maximal dimension among $G_{\alpha}$-orbits in the irreducible variety $\mathcal{O}_{M} \star \mathcal{O}_{N}$ (Lemma 2.2). This orbit $\mathcal{O}_{L}$ is open and dense in $\mathcal{O}_{M} \star \mathcal{O}_{N}$. Thus $\mathcal{O}_{L^{\prime}} \subseteq \overline{\mathcal{O}}_{L}$ for any extension $L^{\prime}$ of $M$ by $N$, i.e., $L$ is the unique maximal element under the partial order $\preceq$ among all extensions of $M$ by $N$. This $L$ is called the generic extension of $M$ by $N$ and is denoted by $L=M \diamond N$. (See [Re] for the finite type cases.) If we define a binary operation in $\mathcal{P}$ by $[M] \diamond[N]=[M \diamond N]$, then $(\mathcal{P}, \diamond)$ is a monoid with identity [0].

We recall that $\mathcal{O}_{M} \star \mathcal{O}_{N}=p_{3} p_{2} p_{1}^{-1}\left(\mathcal{O}_{M} \times \mathcal{O}_{N}\right)$. Since $p_{1}$ is a locally trivial fibration, $p_{1}^{-1}\left(\mathcal{O}_{M \diamond N} \times \mathcal{O}_{W}\right)$ is open and dense in $p_{1}^{-1}\left(\left(\mathcal{O}_{M} \star \mathcal{O}_{N}\right) \times \mathcal{O}_{W}\right)$ for any $[W] \in \mathcal{P}$. Hence $\mathcal{O}_{M \diamond N} \star \mathcal{O}_{W}$ is dense in $\mathcal{O}_{M} \star \mathcal{O}_{N} \star \mathcal{O}_{W}$. Therefore

$$
\mathcal{O}_{(M \diamond N) \diamond W} \subseteq \mathcal{O}_{M \diamond N} \star \mathcal{O}_{W} \subseteq \mathcal{O}_{M} \star \mathcal{O}_{N} \star \mathcal{O}_{W}
$$

implies that $\mathcal{O}_{(M \diamond N) \diamond W}$ is the unique dense orbit in $\mathcal{O}_{M} \star \mathcal{O}_{N} \star \mathcal{O}_{W}$. This not only proves the associativity of $\diamond$ but also implies that

$$
g_{M, N}^{M \diamond N} g_{M \diamond N, W}^{M \diamond N \diamond W}=g_{M, N, W}^{M \diamond N \diamond W}
$$

Let $\Omega$ be the set of all words on the alphabet set $I$. For each word $w=$ $i_{1} \cdots i_{m} \in \Omega$, we define

$$
M(w)=S_{i_{1}} \diamond \cdots \diamond S_{i_{m}} .
$$

Then there is a unique $\pi \in \Pi$ such that $M(\pi) \simeq M(w)$, and we define $\wp(w)=\pi$. It has been proved in [R2] that $\pi=\wp(w) \in \Pi^{a}$ and $\wp$ is a surjective map $\wp: \Omega \rightarrow \Pi^{a}$.

For each module $M$ in $\mathcal{T}$ and each integer $s \geq 1$, we denote by $s M$ the direct sum of $s$ copies of $M$. For $w \in \Omega$, write $w$ in the tight form $w=j_{1}^{e_{1}} \cdots j_{t}^{e_{t}} \in \Omega$ with $j_{r-1} \neq j_{r}$ for all $r$, and define $\mu_{r} \in \Pi$ such that $M\left(\mu_{r}\right)=e_{r} S_{j_{r}}$. For any $\lambda \in \Pi_{\sum_{r=1}^{t} e_{r} j_{r}}$, write $g_{w}^{\lambda}$ for the Hall polynomial $g_{M\left(\mu_{1}\right), \ldots, M\left(\mu_{t}\right)}^{M(\lambda)}$. A word $w$ is called distinguished if the Hall polynomial $g_{w}^{\wp(w)}$ equals 1 . This means that $M(\wp(w))$ has a unique reduced filtration of type $w$, i.e., a filtration

$$
M(\wp(w))=M_{0} \supset M_{1} \supset \cdots \supset M_{t-1} \supset M_{t}=0
$$

with $M_{r-1} / M_{r} \simeq e_{r} S_{j_{r}}$ for all $r$.

Proposition 3.1. For any $\pi \in \Pi^{a}$, there exists a distinguished word $w_{\pi}=$ $j_{1}^{e_{1}} \cdots j_{t}^{e_{t}} \in \wp^{-1}(\pi)$ in tight form. 
In $\mathcal{H}^{*}$, let $u_{i}^{(* m)}=E_{i}^{(* m)}=u_{i}^{* m} /[m] !, i \in I, m \geq 1$. The $\mathcal{Z}$-subalgebra $\mathcal{C}^{*}=\mathcal{C}_{\mathcal{Z}}^{*}$ of $\prod_{q} \mathcal{H}_{q}^{*}$ generated by $u_{i}^{(* m)}, i \in I, m \geq 1$, is the twisted generic composition algebra of $\mathcal{T}$ (cf. §1.4).

\section{$\S 3.3$}

For each $w=j_{1}^{e_{1}} \cdots j_{t}^{e_{t}} \in \Omega$ in tight form, define the monomial

$$
\mathfrak{m}^{(w)}=E_{j_{1}}^{\left(* e_{1}\right)} * \cdots * E_{j_{t}}^{\left(* e_{t}\right)} \in \mathcal{C}^{*} .
$$

From now on, we fix a distinguished word $w_{\pi} \in \wp^{-1}(\pi)$ for each $\pi \in \Pi^{a}$ and thus a fixed section of distinguished words $\mathcal{D}=\left\{w_{\pi} \mid \pi \in \Pi^{a}\right\}$ of $\wp$ over $\Pi^{a}$. For each distinguished word $w_{\pi}=j_{1}^{e_{1}} \cdots j_{t}^{e_{t}} \in \mathcal{D}$ in tight form, define $L_{1}=e_{j_{1}} S_{j_{1}}$ and $L_{i}=L_{i-1} \diamond e_{j_{i}} S_{j_{i}}$ for $i=2, \ldots, t$. Set $\alpha=\underline{\operatorname{dim}} L_{t}$. Then $M(\pi) \simeq L_{t}$. Since

$$
1=g_{w_{\pi}}^{\pi}=g_{e_{j_{1}} S_{j_{1}}, e_{j_{2}} S_{j_{2}}}^{L_{2}} g_{L_{2}, e_{j_{3}} S_{j_{3}}}^{L_{3}} \cdots g_{L_{t-1}, e_{j_{t}} S_{j_{t}}}^{\pi},
$$

we get $g_{L_{i-1}, e_{i} S_{j_{i}}}^{L_{i}}=1$ for $2 \leq i \leq t$. Furthermore, by Lemma 2.3(vi) and Proposition 1.1, we have

$$
\left\langle L_{i-1}\right\rangle *\left\langle e_{j_{i+1}} S_{j_{i+1}}\right\rangle=\left\langle L_{i}\right\rangle+\sum_{X \prec L_{i}} a_{X}\langle X\rangle
$$

with $a_{X} \in \mathcal{Z}_{v_{q}}$. Recall from $\S 1.2$ that $\langle M\rangle=v_{q}^{-\operatorname{dim} M+\operatorname{dim} \operatorname{End} M} u_{[M]}$. Thus

$$
\mathfrak{m}^{\left(w_{\pi}\right)}=\langle M(\pi)\rangle+\sum_{\lambda \prec \pi} \xi_{w_{\pi}}^{\lambda}\langle M(\lambda)\rangle,
$$

where $\xi_{w_{\pi}}^{\lambda} \in \mathcal{Z}_{v_{q}}$. Note that $\xi_{w_{\pi}}^{\lambda} \neq 0$ implies $\underline{\operatorname{dim}} M(\lambda)=\underline{\operatorname{dim}} M(\pi)=\alpha$. Although $\mathfrak{m}^{\left(w_{\pi}\right)} \in \mathcal{C}_{\mathcal{Z}_{v_{q}}}^{*}$, the terms $\langle M\rangle$ are not necessarily in $\mathcal{C}_{\mathcal{Z}_{v_{q}}}^{*}$. Define $E_{\pi}$ inductively by the relation (noting that $v_{q}^{2}=q$ in each component)

$$
E_{\pi}=\mathfrak{m}^{\left(w_{\pi}\right)}-\sum_{\lambda \prec \pi, \lambda \in \Pi_{\alpha}^{a}} v_{q}^{-\operatorname{dim} M(\pi)+\operatorname{dim} \operatorname{End} M(\pi)+\operatorname{dim} M(\lambda)-\operatorname{dim} \operatorname{End} M(\lambda)} g_{w_{\pi}}^{\lambda}\left(v_{q}^{2}\right) E_{\lambda} .
$$

If $\pi \in \Pi_{\alpha}^{a}$ is minimal, then $E_{\pi}=\mathfrak{m}^{\left(w_{\pi}\right)} \in \mathcal{C}_{\mathcal{Z}_{v_{q}}}^{*}$. By induction on the partial order, we have $E_{\pi} \in \mathcal{C}_{\mathcal{Z}_{v_{q}}}^{*}$ for all $\pi \in \Pi^{a}$. If $M(\pi)=S_{i}$, then $E_{\pi}=E_{i}=u_{\left[S_{i}\right]}$. Therefore

$$
E_{\pi}=\langle M(\pi)\rangle+\sum_{\lambda \in \Pi_{\alpha} \backslash \Pi_{\alpha}^{a}, \lambda \prec \pi} \eta_{\lambda}^{\pi}\langle M(\lambda)\rangle
$$

with $\eta_{\lambda}^{\pi} \in \mathcal{Z}_{v_{q}}$. By applying the genericalization process in $\S 1.4$, we have 
Proposition 3.2. Let $\mathcal{D}=\left\{w_{\pi} \mid \pi \in \Pi^{a}\right\}$ be a section of distinguished words of $\Omega$ over $\Pi^{a}$. Then both $\left\{\mathfrak{m}^{\left(w_{\pi}\right)} \mid \pi \in \Pi^{a}\right\}$ and $\left\{E_{\pi} \mid \pi \in \Pi^{a}\right\}$ are $\mathcal{Z}$-bases of $\mathcal{C}_{\mathcal{Z}}^{*}$. Furthermore, for any $\pi \in \Pi_{\alpha}^{a}$,

$\mathfrak{m}^{\left(w_{\pi}\right)}=E_{\pi}+\sum_{\lambda \in \Pi_{\alpha}^{\alpha}, \lambda \prec \pi} v^{-\operatorname{dim} M(\pi)+\operatorname{dim} \operatorname{End} M(\pi)+\operatorname{dim} M(\lambda)-\operatorname{dim} \operatorname{End} M(\lambda)} g_{w_{\pi}}^{\lambda}\left(v^{2}\right) E_{\lambda}$.

Remark. The definition of the basis $\left\{E_{\pi} \mid \pi \in \Pi^{a}\right\}$ relies on the choice of the section $\mathcal{D}$ of distinguished words. However it is proved in [DDX] that this basis is independent of the choice of $\mathcal{D}$.

We will call $\left\{\mathfrak{m}^{\left(w_{\pi}\right)} \mid \pi \in \Pi^{a}\right\}$ a monomial $\mathcal{Z}$-basis of $\mathcal{C}_{\mathcal{Z}}^{*}$ and $\left\{E_{\pi} \mid \pi \in \Pi^{a}\right\}$ a $P B W$ basis of $\mathcal{C}_{\mathcal{Z}}^{*}$. With the triangular relation between the two bases, we can follow the approach of Lusztig [L1, 7.8-7.11] to obtain the canonical basis $\left\{\mathcal{E}_{\pi} \mid \pi \in \Pi^{a}\right\}$ of $\mathcal{C}_{\mathcal{Z}}^{*}$ in the sense of $[\mathrm{L} 1,3.1]$ by

$$
\mathcal{E}_{\pi}=\sum_{\lambda \preceq \pi, \lambda \in \Pi_{\alpha}^{\alpha}} p_{\lambda \pi} E_{\lambda} \quad \text { for } \pi \in \Pi_{\alpha}^{a}, \alpha \in \mathbb{N}^{n}
$$

with $p_{\lambda \lambda}=1$ and $p_{\lambda \pi} \in v^{-1} \mathbb{Z}\left[v^{-1}\right]$ for $\lambda \prec \pi$.

The following lemma will be used in Section 6 .

Lemma 3.3. For any $l \in \mathbb{N}$ and $1 \leq j \leq n$, let $\pi, \pi^{\prime} \in \Pi^{a}$ be such that $S_{j}[l]=$ $M(\pi)$ and $S_{j+1}[l]=M\left(\pi^{\prime}\right)$. Then

(i) $\quad u_{\left[S_{j}[l]\right]} \equiv \sum_{\lambda \preceq \pi, \lambda \in \Pi^{a}} a_{\lambda} E_{\lambda}\left(\bmod (v-1) \mathcal{C}_{\mathcal{Z}}^{*}\right) \quad$ if $n \nmid l$,

(ii) $\quad u_{\left[S_{j}[l]\right]}-u_{\left[S_{j+1}[l]\right]} \equiv \sum_{\lambda \preceq \pi\left(o r \pi^{\prime}\right), \lambda \in \Pi^{a}} a_{\lambda} E_{\lambda}\left(\bmod (v-1) \mathcal{C}_{\mathcal{Z}}^{*}\right) \quad$ if $n \mid l$.

Here $a_{\lambda} \in \mathbb{Q}$.

Proof. We use induction on $l$ and assume $j=1$ for each $l$. For $l=1$, we have $u_{\left[S_{1}\right]}=E_{1}$ and the conclusion follows. Assume that $2 \leq l \leq n-1$ and the conclusion is true for $\pi_{1}$ such that $M\left(\pi_{1}\right)=S_{1}[l-1]$. Then

$$
u_{\left[S_{1}[l-1]\right]} \equiv \sum_{\lambda \preceq \pi_{1}, \lambda \in \Pi^{a}} a_{\lambda} E_{\lambda}\left(\bmod (v-1) \mathcal{C}_{\mathcal{Z}}^{*}\right)
$$

and $u_{\left[S_{1}[l-1]\right]} * u_{\left[S_{l}\right]}-u_{\left[S_{l}\right]} * u_{\left[S_{1}[l-1]\right]} \equiv u_{\left[S_{1}[l]\right]}\left(\bmod (v-1) \mathcal{C}_{\mathcal{Z}}^{*}\right)$. For $\lambda \preceq \pi_{1}$ and $\lambda \in \Pi^{a}$, we have $\mathcal{O}_{M(\lambda)} \subseteq \overline{\mathcal{O}}_{M\left(\pi_{1}\right)}=\overline{\mathcal{O}}_{S_{1}[l-1]}$. Thus

$$
\mathcal{O}_{M(\lambda) \diamond S_{l}} \subseteq \overline{\mathcal{O}}_{M\left(\pi_{1}\right) \diamond S_{l}}=\overline{\mathcal{O}}_{M(\pi)}=\overline{\mathcal{O}}_{S_{1}[l]},
$$


and

$$
\begin{aligned}
u_{\left[S_{1}[l]\right]} & \equiv\left(\sum_{\lambda \preceq \pi_{1}, \lambda \in \Pi^{a}} a_{\lambda} E_{\lambda}\right) * E_{l}-E_{l} *\left(\sum_{\lambda \preceq \pi_{1}, \lambda \in \Pi^{a}} a_{\lambda} E_{\lambda}\right)\left(\bmod (v-1) \mathcal{C}_{\mathcal{Z}}^{*}\right) \\
& \equiv\left(\sum_{\lambda \preceq \pi_{1}, \lambda \in \Pi^{a}} a_{\lambda} E_{\lambda}\right) * E_{l}-E_{l} *\left(\sum_{\lambda \preceq \pi_{1}, \lambda \in \Pi^{a}} a_{\lambda} E_{\lambda}\right) \equiv \sum_{\lambda \preceq \pi, \lambda \in \Pi^{a}} a_{\lambda}^{\prime} E_{\lambda}
\end{aligned}
$$

since $\left\{E_{\lambda} \mid \lambda \in \Pi^{a}\right\}$ is a $\mathcal{Z}$-basis of $\mathcal{C}_{\mathcal{Z}}^{*}$. Thus the conclusion is true for $l$. For $l=n$, by induction assumption (for $j=2$ ), we have, with $M\left(\pi_{2}\right)=S_{2}[l-1]$,

$$
\begin{gathered}
u_{\left[S_{2}[l-1]\right]} \equiv \sum_{\lambda \preceq \pi_{2}, \lambda \in \Pi^{a}} a_{\lambda} E_{\lambda}\left(\bmod (v-1) \mathcal{C}_{\mathcal{Z}}^{*}\right) \\
u_{\left[S_{1}[l]\right]}-u_{\left[S_{2}[l]\right]} \equiv u_{\left[S_{1}\right]} * u_{\left[S_{2}[l-1]\right]}-u_{\left[S_{2}[l-1]\right]} * u_{\left[S_{1}\right]}\left(\bmod (v-1) \mathcal{C}_{\mathcal{Z}}^{*}\right) \\
\equiv E_{1}\left(\sum_{\lambda \preceq \pi_{2}, \lambda \in \Pi^{a}} a_{\lambda} E_{\lambda}\right)-\left(\sum_{\lambda \preceq \pi_{2}, \lambda \in \Pi^{a}} a_{\lambda} E_{\lambda}\right) * E_{1}\left(\bmod (v-1) \mathcal{C}_{\mathcal{Z}}^{*}\right) \\
\equiv \sum_{\lambda \preceq \pi\left(\operatorname{or} \pi^{\prime}\right), \lambda \in \Pi^{a}} a_{\lambda}^{\prime} E_{\lambda}\left(\bmod (v-1) \mathcal{C}_{\mathcal{Z}}^{*}\right) .
\end{gathered}
$$

Now we consider the general case. Let $l=k n+m, 0<m \leq n-1$. We set $M\left(\pi_{1}\right)=S_{1}[l-1]$ and $M\left(\pi_{1}^{\prime}\right)=S_{2}[l-1]$. If $m=1$, by induction assumption, we have

$$
u_{\left[S_{1}[l-1]\right]}-u_{\left[S_{2}[l-1]\right]} \equiv \sum_{\lambda \preceq \pi_{1}\left(\text { or } \pi_{1}^{\prime}\right), \lambda \in \Pi^{a}} a_{\lambda} E_{\lambda}\left(\bmod (v-1) \mathcal{C}_{\mathcal{Z}}^{*}\right)
$$

Hence

$$
\begin{aligned}
u_{\left[S_{1}[l]\right]} & \equiv\left(u_{\left[S_{1}[l-1]\right]}-u_{\left[S_{2}[l-1]\right]}\right) * u_{\left[S_{1}\right]}-u_{\left[S_{1}\right]} *\left(u_{\left[S_{1}[l-1]\right]}-u_{\left[S_{2}[l-1]\right]}\right) \\
& \equiv\left(\sum_{\lambda \preceq \pi_{1}\left(\text { or } \pi_{1}^{\prime}\right), \lambda \in \Pi^{a}} a_{\lambda} E_{\lambda}\right) * E_{1}-E_{1} *\left(\sum_{\lambda \preceq \pi_{1}\left(\text { or } \pi_{1}^{\prime}\right), \lambda \in \Pi^{a}} a_{\lambda} E_{\lambda}\right) \\
& \equiv \sum_{\lambda \preceq \pi, \lambda \in \Pi^{a}} a_{\lambda}^{\prime} E_{\lambda}\left(\bmod (v-1) \mathcal{C}_{\mathcal{Z}}^{*}\right) .
\end{aligned}
$$

Here in the last part we used the fact that $l-1=n k$ and $S_{1}[l]$ is the unique indecomposable module of dimension vector $\underline{\operatorname{dim}} S_{1}[l]$ which is a real root. All $E_{\lambda}$ appearing in the sum have terms $u_{[M]}$ in $\mathcal{H}^{*}$ with $\underline{\operatorname{dim}} M=\underline{\operatorname{dim}} S_{1}[l]$. Since $S_{1}[l]$ has no self-extension, $O_{S_{1}[l]}$ is an open dense orbit.

If $2 \leq m \leq n-1$, the argument is the same as in the case $2 \leq l \leq n-1$. When $n \mid l$, i.e., $m=0$, the argument is the same as for $l=n$. 


\section{§4. Integral bases arising from preprojective and preinjective components}

In this section we consider a connected tame quiver $Q$ without oriented cycles. For the preprojective and preinjective components, the argument in this section is essentially the same as in the case of finite type.

\section{$\S 4.1$}

Recall that $U$ is the quantized affine enveloping algebra over $\mathbb{Q}(v)$ associated to the quiver $Q$, with the Chevalley generators $E_{i}, F_{i}$ and $K_{i}^{ \pm}$. Lusztig [L5] introduced the symmetries $T_{i, 1}^{\prime \prime}: U \rightarrow U$ for $i \in I$ as algebra automorphisms of $U$ defined by

$$
\begin{aligned}
& T_{i, 1}^{\prime \prime}\left(K_{\beta}\right)=K_{s_{i}(\beta)}, \quad T_{i, 1}^{\prime \prime}\left(E_{i}\right)=-F_{i} K_{i}, \quad T_{i, 1}^{\prime \prime}\left(F_{i}\right)=-K_{i} E_{i}, \\
& T_{i, 1}^{\prime \prime}\left(E_{j}\right)=\sum_{r+s=-a_{i j}}(-1)^{r} v^{-r} E_{i}^{(s)} E_{j} E_{i}^{(r)} \quad \text { for } j \neq i \text { in } I, \\
& T_{i, 1}^{\prime \prime}\left(F_{j}\right)=\sum_{r+s=-a_{i j}}(-1)^{r} v^{r} F_{i}^{(r)} F_{j} F_{i}^{(s)} \quad \text { for } j \neq i \text { in } I .
\end{aligned}
$$

Here $a_{i j}=(i, j)$ for $i, j \in I$, and $s_{i}(\beta)=\beta-(\beta, i) i$ for $\beta \in \mathbb{Z} I$. For each $i \in I$, define

$$
U^{+}[i]=\left\{x \in U^{+} \mid T_{i, 1}^{\prime \prime}(x) \in U^{+}\right\},
$$

which is a $\mathbb{Q}(v)$-subalgebra of $U^{+}$. Then $T_{i, 1}^{\prime \prime}: U^{+}[i] \rightarrow U^{+}[i]$ is a $\mathbb{Q}(v)$-algebra automorphism. Moreover, if we consider the Lusztig form $U_{\mathcal{Z}}^{+}$and let $U_{\mathcal{Z}}^{+}[i]=$ $U_{\mathcal{Z}}^{+} \cap U^{+}[i]$, then $T_{i, 1}^{\prime \prime}: U_{\mathcal{Z}}^{+}[i] \rightarrow U_{\mathcal{Z}}^{+}[i]$ is a $\mathcal{Z}$-algebra automorphism.

\section{$\S 4.2$}

We define $\sigma_{i} Q$ to be the quiver obtained from $Q$ by reversing the direction of every arrow adjacent to the vertex $i$ and keeping all other arrows unchanged. If $i$ is a sink of $Q$, one can define the BGP reflection functor (see [BGP] or [DR])

$$
\sigma_{i}^{+}: \bmod \Lambda \rightarrow \bmod \sigma_{i} \Lambda
$$

where $\Lambda=\mathbb{F}_{q}(Q)$ and $\sigma_{i} \Lambda=\mathbb{F}_{q}\left(\sigma_{i} Q\right)$ are path algebras. Let $\bmod \Lambda[i]$ be the full subcategory of $\bmod \Lambda$ consisting of all representations which do not have $S_{i}$ as a direct summand. Let $\mathcal{H}^{*}(\Lambda)[i]$ be the subalgebra of $\mathcal{H}^{*}(\Lambda)$ generated by $u_{[M]}$ with $M \in \bmod \Lambda[i]$. Then $\sigma_{i}^{+}$induces a category equivalence $\bmod \Lambda[i] \rightarrow \bmod \sigma_{i}^{+} \Lambda[i]$ and therefore we have an algebra homomorphism

$$
\sigma_{i}: \mathcal{H}^{*}(\Lambda)[i] \rightarrow \mathcal{H}^{*}\left(\sigma_{i} \Lambda\right)[i]
$$


defined by

$$
\sigma_{i}\left(u_{[M]}\right)=u_{\left[\sigma_{i}^{+}(M)\right]} \quad \text { for any } M \in \bmod \Lambda[i] .
$$

Under the identification of $\mathcal{C}_{\mathcal{Z}}^{*}(\Lambda)$ with $U_{\mathcal{Z}}^{+}$, the subalgebra $\mathcal{C}^{*}(\Lambda)_{\mathcal{Z}}[i]=$ $\left\{x \in \mathcal{C}^{*}(\Lambda)_{\mathcal{Z}} \mid \sigma_{i}(x) \in \mathcal{C}^{*}\left(\sigma_{i} \Lambda\right)_{\mathcal{Z}}\right\}$ is identified with $U_{\mathcal{Z}}^{+}[i]$. Similarly $\mathcal{C}_{\mathcal{Z}}^{*}\left(\sigma_{i} \Lambda\right)[i]$ is identified with $U_{\mathcal{Z}}^{+}[i]$.

On the other hand, the functor $\sigma_{i}^{+}$induces an algebra homomorphism

$$
\sigma_{i}: \mathcal{C}^{*}(\Lambda)_{\mathcal{Z}}[i] \rightarrow \mathcal{C}^{*}\left(\sigma_{i} \Lambda\right)_{\mathcal{Z}}[i]
$$

and we have a commutative diagram

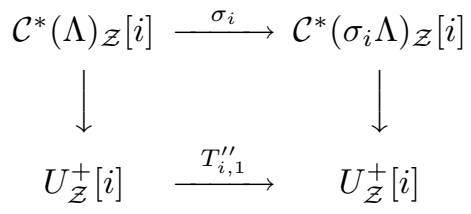

with the vertical maps being the above mentioned identifications (for example, see $[\mathrm{XY}])$.

Dually, if $i$ is a source of $Q$, we have similar results.

We recall that an indecomposable $\Lambda$-module $M$ is exceptional if $\operatorname{Ext}_{\Lambda}^{1}(M, M)$ $=0$. In this case one has $\langle M\rangle^{(* s)}=\langle s M\rangle$ for all $s \geq 1$. It is proved in [CX] that $\langle s M\rangle \in \mathcal{C}^{*}(\Lambda)_{\mathcal{Z}}$ for any exceptional indecomposable $M$ and any $s \geq 1$.

In $[\mathrm{CB}]$, the structure of the Auslander-Reiten quivers of tame quivers is discussed. Indecomposable modules are divided into three classes: preprojective, regular, and preinjective. Regular modules are further divided into homogeneous and non-homogeneous ones, depending on the period being 1 or larger than 1 under the Auslander-Reiten translation.

We denote by Prep and Prei, respectively, the isomorphism classes of indecomposable preprojective and preinjective $\Lambda$-modules. In particular, $\mathcal{C}_{\mathcal{Z}}^{*}$ contains the set

$\left\{\left\langle u_{[s M]}\right\rangle \mid M\right.$ is indecomposable in Prep or Prei and $\left.s \geq 1\right\}$.

\section{$\S 4.3$}

Let $i_{m}, \ldots, i_{1}$ be an admissible sink sequence of $Q$, i.e., $i_{m}$ is a $\operatorname{sink}$ of $Q$ and $i_{t}$ is a sink of the quiver $\sigma_{i_{t+1}} \cdots \sigma_{i_{m}} Q$ for $1 \leq t<m$. Let $M$ be in Prei. There exists an admissible sink sequence of $Q$ such that

$$
M=\sigma_{i_{1}}^{+} \cdots \sigma_{i_{m}}^{+}\left(S_{i_{m+1}}\right),
$$


where $S_{i_{m+1}}$ is a simple representation in $\bmod \sigma_{i_{m}} \cdots \sigma_{i_{1}} \Lambda$. The following lemma can be found in [R3].

Lemma 4.1. Let $M$ be an indecomposable preinjective representation. Then

$$
\langle M\rangle=T_{i_{1}, 1}^{\prime \prime} \cdots T_{i_{m}, 1}^{\prime \prime}\left(E_{i_{m+1}}\right),
$$

where $M=\sigma_{i_{1}}^{+} \cdots \sigma_{i_{m}}^{+}\left(S_{i_{m+1}}\right)$, for an admissible sink sequence $i_{m}, \ldots, i_{1}$ of $Q$.

For each positive real root $\beta$ of the root datum corresponding to the tame quiver $Q$, there is exactly one indecomposable module $M(\beta)$ with $\underline{\operatorname{dim}} M=\beta$. Since Prei is representation-directed, we can give a total order $\leq$ on the set

$$
\Phi_{\text {Prei }}^{+}=\left\{\cdots<\beta_{2}<\beta_{1}\right\}
$$

of all positive real roots $\beta_{i}$ such that $\left[M\left(\beta_{i}\right)\right] \in$ Prei and

$$
\operatorname{Hom}\left(M\left(\beta_{i}\right), M\left(\beta_{j}\right)\right) \neq 0 \quad \text { implies } \quad \beta_{i} \leq \beta_{j} .
$$

Then this order $\leq$ has the property

$$
\left\langle\beta_{i}, \beta_{j}\right\rangle>0 \text { implies } \beta_{i} \leq \beta_{j} \quad \text { and } \quad\left\langle\beta_{i}, \beta_{j}\right\rangle<0 \text { implies } \beta_{j}<\beta_{i}
$$

and

$$
\operatorname{Ext}\left(M\left(\beta_{i}\right), M\left(\beta_{j}\right)\right)=0 \quad \text { for } \beta_{i} \leq \beta_{j} .
$$

Similarly, since Prep is representation-directed, we define a total order $\leq$ on the set

$$
\Phi_{\text {Prep }}^{+}=\left\{\alpha_{1}<\alpha_{2}<\cdots\right\}
$$

of all positive real roots $\alpha_{i}$ such that $\left[M\left(\alpha_{i}\right)\right] \in$ Prep and

$$
\operatorname{Hom}\left(M\left(\alpha_{i}\right), M\left(\alpha_{j}\right)\right) \neq 0 \quad \text { implies } \quad \alpha_{i} \leq \alpha_{j} .
$$

Then this order has the property

$$
\left\langle\alpha_{i}, \alpha_{j}\right\rangle>0 \text { implies } \alpha_{i} \leq \alpha_{j} \text { and }\left\langle\alpha_{i}, \alpha_{j}\right\rangle<0 \text { implies } \alpha_{j}<\alpha_{i}
$$

and

$$
\operatorname{Ext}\left(M\left(\alpha_{i}\right), M\left(\alpha_{j}\right)\right)=0 \quad \text { for } \alpha_{i} \leq \alpha_{j}
$$

We denote by $\mathbb{N}_{f}^{P r e i}$ the set of all support-finite functions $\mathbf{b}: \Phi_{\text {Prei }}^{+} \rightarrow \mathbb{N}$. Each $\mathbf{b} \in \mathbb{N}_{f}^{P r e i}$ defines a preinjective representation

$$
M(\mathbf{b})=\bigoplus_{\beta_{i} \in \Phi_{\text {Prei }}^{+}} \mathbf{b}\left(\beta_{i}\right) M\left(\beta_{i}\right)
$$


and any preinjective representation is isomorphic to exactly one of the form $M(\mathbf{b})$. By Ringel [R3, Prop. 1] we have

Lemma 4.2. For any $\mathbf{b} \in \mathbb{N}_{f}^{\text {Prei }}$,

$$
\langle M(\mathbf{b})\rangle=\left\langle\mathbf{b}\left(\beta_{i_{m}}\right) M\left(\beta_{i_{m}}\right)\right\rangle * \cdots *\left\langle\mathbf{b}\left(\beta_{i_{1}}\right) M\left(\beta_{i_{1}}\right)\right\rangle,
$$

where $\left\{\beta_{i_{m}}<\cdots<\beta_{i_{1}}\right\}$ is the support of $\mathbf{b}$ in $\Phi_{\text {Prei }}^{+}$.

Thus, by discussions in $\S 4.2,\langle M(\mathbf{b})\rangle \in \mathcal{C}_{\mathcal{Z}}^{*}$ for all $\mathbf{b} \in \mathbb{N}_{f}^{\text {Prei }}$. We now define $\mathcal{C}^{*}($ Prei $)$ to be the $\mathcal{Z}$-submodule of $\mathcal{C}_{\mathcal{Z}}^{*}$ generated by $\left\{\langle M(\mathbf{b})\rangle \mid \mathbf{b} \in \mathbb{N}_{f}^{\text {Prei }}\right\}$.

Lemma 4.3. The $\mathcal{Z}$-submodule $\mathcal{C}^{*}($ Prei $)$ is a subalgebra of $\mathcal{C}_{\mathcal{Z}}^{*}$ and $\{\langle M(\mathbf{b})\rangle \mid$ $\mathbf{b} \in \mathbb{N}_{f}^{\text {Prei }\}}$ is a $\mathcal{Z}$-basis of $\mathcal{C}^{*}($ Prei $)$.

Proof. If $\mathbf{b}, \mathbf{b}_{1}, \mathbf{b}_{2} \in \mathbb{N}_{f}^{\text {Prei }}$, then the Hall polynomial $g_{M\left(\mathbf{b}_{1}\right) M\left(\mathbf{b}_{2}\right)}^{M(\mathbf{b})}$ always exists (see Ringel [R5]). Then it is easy to see that $\mathcal{C}^{*}($ Prei) is closed under the multiplication $*$.

With similar definitions for Prep, we have

Lemma 4.4. For any $\mathbf{a} \in \mathbb{N}_{f}^{\text {Prep }}$ and $M(\mathbf{a})=\bigoplus_{\alpha_{i} \in \Phi_{\text {Prep }}^{+}} \mathbf{a}\left(\alpha_{i}\right) M\left(\alpha_{i}\right)$,

$$
\langle M(\mathbf{a})\rangle=\left\langle\mathbf{a}\left(\alpha_{i_{1}}\right) M\left(\alpha_{i_{1}}\right)\right\rangle * \cdots *\left\langle\mathbf{a}\left(\alpha_{i_{m}}\right) M\left(\alpha_{i_{m}}\right)\right\rangle,
$$

where $\left\{\alpha_{i_{1}}<\cdots<\alpha_{i_{m}}\right\}$ is the support of $\mathbf{a}$ in $\Phi_{\text {Prep }}^{+}$.

Lemma 4.5. Let $\mathcal{C}^{*}($ Prep $)$ be the $\mathcal{Z}$-submodule of $\mathcal{C}_{\mathcal{Z}}^{*}$ generated by $\{\langle M(\mathbf{a})\rangle \mid$ $\left.\mathbf{a} \in \mathbb{N}_{f}^{\text {Prep }}\right\}$. Then $\mathcal{C}^{*}($ Prep $)$ is a subalgebra of $\mathcal{C}_{\mathcal{Z}}^{*}$ and $\left\{\langle M(\mathbf{a})\rangle \mid \mathbf{a} \in \mathbb{N}_{f}^{\text {Prep }}\right\}$ is its $\mathcal{Z}$-basis.

\section{$\S 4.4$}

Since $Q$ is a tame quiver without oriented cycles, we can order the set $\left\{S_{1}, \ldots, S_{n}\right\}$ of non-isomorphic nilpotent simple modules in $\bmod \Lambda$ so that

$$
\operatorname{Ext}^{1}\left(S_{i}, S_{j}\right)=0 \quad \text { for } i \geq j .
$$

We can now identify $I=\{1, \ldots, n\}$ and $\mathbb{N} I=\mathbb{N}^{n}$ so that $S_{i}$ is the simple module at the vertex $i \in I$. Any module $M$ with dimension vector $\mathbf{d}=\left(d_{1}, \ldots, d_{n}\right)$ has a unique filtration

$$
M=M_{0} \supseteq M_{1} \supseteq \cdots \supseteq M_{n}=0
$$

with factors $M_{i-1} / M_{i}$ isomorphic to $d_{i} S_{i}$, since $\operatorname{Ext}^{1}\left(S_{i}, S_{j}\right)=0$ for $i \geq j$. This shows that the Hall polynomial $g_{d_{1} S_{1} \cdots d_{n} S_{n}}^{M}$ equals 1 . By setting

$$
\psi_{n}(q)=\frac{(1-q) \cdots\left(1-q^{n}\right)}{(1-q)^{n}},
$$


we have, in $\mathcal{H}_{q}$ and $\mathcal{H}_{q}^{*}$ respectively,

$$
\begin{gathered}
u_{\left[S_{1}\right]}^{d_{1}} \cdots u_{\left[S_{n}\right]}^{d_{n}}=\psi_{d_{1}}(q) \cdots \psi_{d_{n}}(q) \sum u_{[M(\mathbf{a}) \oplus M(\mathbf{t}) \oplus M(\mathbf{b})]}, \\
u_{\left[S_{1}\right]}^{\left(* d_{1}\right)} * \cdots * u_{\left[S_{n}\right]}^{\left(* d_{n}\right)}=v_{q}^{-\left(d_{1}+\cdots+d_{n}\right)+\langle\mathbf{d}, \mathbf{d}\rangle} \sum u_{[M(\mathbf{a}) \oplus M(\mathbf{t}) \oplus M(\mathbf{b})]},
\end{gathered}
$$

where the summation is over the triples $(M(\mathbf{a}), M(\mathbf{t}), M(\mathbf{b}))$ with $M(\mathbf{a})$ preprojective, $M(\mathbf{t})$ regular, $M(\mathbf{b})$ preinjective, and $\underline{\operatorname{dim}} M(\mathbf{a})+\underline{\operatorname{dim}} M(\mathbf{t})+\underline{\operatorname{dim}} M(\mathbf{b})=$ $\left(d_{1}, \ldots, d_{n}\right)=\mathbf{d}$.

For any $\mathbf{a} \in \mathbb{N}_{f}^{\text {Prep }}$, let $\left\{\alpha_{i_{1}}<\cdots<\alpha_{i_{m}}\right\}$ be the support of $\mathbf{a}$ and, for $1 \leq t \leq m$, define

$$
\begin{aligned}
\mathbf{a}_{t} & =\mathbf{a}\left(\alpha_{i_{t}}\right) \alpha_{i_{t}}=\left(a_{1 t}, \ldots, a_{n t}\right) \in \mathbb{N}^{n} \\
\mathfrak{m}_{\mathbf{a}_{t}} & =u_{\left[S_{1}\right]}^{\left(* a_{1 t}\right)} * * \cdots * u_{\left[S_{n}\right]}^{\left(* a_{n}\right)}, \quad \mathfrak{m}_{\mathbf{a}}=\mathfrak{m}_{\mathbf{a}_{1}} * \cdots * \mathfrak{m}_{\mathbf{a}_{m}} .
\end{aligned}
$$

Similarly for $\mathbf{b} \in \mathbb{N}_{f}^{P r e i}$ with support $\left\{\beta_{i_{1}}<\cdots<\beta_{i_{m}}\right\}$ define

$$
\mathfrak{m}_{\mathbf{b}_{t}}=u_{\left[S_{1}\right]}^{\left(* b_{11}\right)} * \cdots * u_{\left[S_{n}\right]}^{\left(* b_{n t}\right)}, \quad \mathfrak{m}_{\mathbf{b}}=\mathfrak{m}_{\mathbf{b}_{m}} * \cdots * \mathfrak{m}_{\mathbf{b}_{1}} .
$$

Lemma 4.6. For any $\mathbf{a} \in \mathbb{N}_{f}^{\text {Prep }}$ and $\mathbf{b} \in \mathbb{N}_{f}^{\text {Prei }}$, we have, in $\mathcal{H}^{*}$,

(i) $\quad \mathfrak{m}_{\mathbf{a}}=\langle M(\mathbf{a})\rangle+\sum_{\operatorname{dim} \mathcal{O}_{M\left(\mathbf{a}^{\prime}\right) \oplus M\left(\mathbf{t}^{\prime}\right) \oplus M\left(\mathbf{b}^{\prime}\right)}<\operatorname{dim} \mathcal{O}_{M(\mathbf{a})}} c_{\mathbf{a}^{\prime} \mathbf{t}^{\prime} \mathbf{b}^{\prime}}^{\mathbf{a}}\left(v_{q}\right) u_{\left[M\left(\mathbf{a}^{\prime}\right) \oplus M\left(\mathbf{t}^{\prime}\right) \oplus M\left(\mathbf{b}^{\prime}\right)\right]}$.

Here the sum ranges over all triples $M\left(\mathbf{a}^{\prime}\right), M\left(\mathbf{t}^{\prime}\right), M\left(\mathbf{b}^{\prime}\right)$ with $M\left(\mathbf{a}^{\prime}\right)$ preprojective, $M\left(\mathbf{t}^{\prime}\right)$ regular, $M\left(\mathbf{b}^{\prime}\right)$ preinjective, and $\underline{\operatorname{dim}} M\left(\mathbf{a}^{\prime}\right)+\underline{\operatorname{dim}} M\left(\mathbf{t}^{\prime}\right)+\underline{\operatorname{dim}} M\left(\mathbf{b}^{\prime}\right)=$ $\sum_{\alpha \in \text { Prep }} \mathbf{a}(\alpha) \alpha$, and $c_{\mathbf{a}^{\prime} \mathbf{t}^{\prime} \mathbf{b}^{\prime}}^{\mathbf{a}}(v) \in \mathbb{Z}\left[v, v^{-1}\right]$. Moreover

(ii) $\mathfrak{m}_{\mathbf{b}}=\langle M(\mathbf{b})\rangle$

$$
+\sum_{\operatorname{dim} \mathcal{O}_{M\left(\mathbf{a}^{\prime \prime}\right) \oplus M\left(\mathbf{t}^{\prime \prime}\right) \oplus M\left(\mathbf{b}^{\prime \prime}\right)}<\operatorname{dim} \mathcal{O}_{M(\mathbf{b})}} d_{\mathbf{a}^{\prime \prime} \mathbf{t}^{\prime \prime} \mathbf{b}^{\prime \prime}}^{\mathbf{b}}\left(v_{q}\right) u_{\left[M\left(\mathbf{a}^{\prime \prime}\right) \oplus M\left(\mathbf{t}^{\prime \prime}\right) \oplus M\left(\mathbf{b}^{\prime \prime}\right)\right]},
$$

where the sum is over all triples $M\left(\mathbf{a}^{\prime \prime}\right), M\left(\mathbf{t}^{\prime \prime}\right), M\left(\mathbf{b}^{\prime \prime}\right)$ with $M\left(\mathbf{a}^{\prime \prime}\right)$ preprojective, $M\left(\mathbf{t}^{\prime \prime}\right)$ regular, $M\left(\mathbf{b}^{\prime \prime}\right)$ preinjective, and $\underline{\operatorname{dim}} M\left(\mathbf{a}^{\prime \prime}\right)+\underline{\operatorname{dim}} M\left(\mathbf{t}^{\prime \prime}\right)+\underline{\operatorname{dim}} M\left(\mathbf{b}^{\prime \prime}\right)=$ $\sum_{\beta \in \operatorname{Prep}} \mathbf{b}(\beta) \beta$, and $d_{\mathbf{a}^{\prime \prime} \mathbf{t}^{\prime \prime} \mathbf{b}^{\prime \prime}}(v) \in \mathbb{Z}\left[v, v^{-1}\right]$.

Proof. (i) Since $M\left(\alpha_{i_{t}}\right)$ is exceptional, by Lemma 2.1, $\mathcal{O}_{\mathbf{a}\left(\alpha_{i_{t}}\right) M\left(\alpha_{i_{t}}\right)}$ is a unique orbit of maximal dimension in $\mathbb{E}_{\mathbf{a}\left(\alpha_{i_{t}}\right) \alpha_{i_{t}}}$. Note that all simple modules are excep- 
tional. We have

$$
\begin{aligned}
& \mathfrak{m}_{\mathbf{a}_{t}}=u_{\left[S_{1}\right]}^{\left(* a_{1 t}\right)} * \cdots * u_{\left[S_{n}\right]}^{\left(* a_{n t}\right)}=\left\langle a_{1 t} S_{1}\right\rangle * \cdots *\left\langle a_{n t} S_{n}\right\rangle \\
& =v_{q}^{-\operatorname{dim}\left(\mathbf{a}\left(\alpha_{i_{t}}\right) M\left(\alpha_{i_{t}}\right)\right)+\operatorname{dim} \operatorname{End}\left(\mathbf{a}\left(\alpha_{i_{t}}\right) M\left(\alpha_{i_{t}}\right)\right)} \sum_{\underline{\operatorname{dim}} M=\mathbf{a}\left(\alpha_{i_{t}}\right) \alpha_{i_{t}}} u_{[M]} \\
& =\left\langle\mathbf{a}\left(\alpha_{i_{t}}\right) M\left(\alpha_{i_{t}}\right)\right\rangle+\sum_{\operatorname{dim} \mathcal{O}_{M}<\operatorname{dim} \mathcal{O}_{\mathbf{a}\left(\alpha_{i_{t}}\right) M\left(\alpha_{i_{t}}\right)}} v_{q}^{-\operatorname{dim} \operatorname{Ext}(M, M)}\langle M\rangle .
\end{aligned}
$$

Because $\operatorname{Ext}\left(M\left(\alpha_{i_{t}}\right), M\left(\alpha_{i_{s}}\right)\right)=0$ and $\operatorname{Hom}\left(M\left(\alpha_{i_{s}}\right), M\left(\alpha_{i_{t}}\right)\right)=0$ for $i_{t}<i_{s}$, by Lemmas 2.3(vii) and $\S 4.4$ we have

$$
\begin{aligned}
\mathfrak{m}_{\mathbf{a}} & =\mathfrak{m}_{\mathbf{a}_{1}} * \mathfrak{m}_{\mathbf{a}_{m}} \\
& =\langle M(\mathbf{a})\rangle+\sum_{\operatorname{dim} \mathcal{O}_{M\left(\mathbf{a}^{\prime}\right) \oplus M\left(\mathbf{t}^{\prime}\right) \oplus M\left(\mathbf{b}^{\prime}\right)}<\operatorname{dim} \mathcal{O}_{M(\mathbf{a})}} c_{\mathbf{a}^{\prime} \mathbf{t}^{\prime} \mathbf{b}^{\prime} q}^{\mathbf{a}} u_{\left[M\left(\mathbf{a}^{\prime}\right) \oplus M\left(\mathbf{t}^{\prime}\right) \oplus M\left(\mathbf{b}^{\prime}\right)\right]},
\end{aligned}
$$

as desired. The proof for (ii) is similar.

Remark. By Lemma 1.2, the degree of $v_{q}^{-1}$ in $c_{\mathbf{a}^{\prime} \mathbf{t}^{\prime} \mathbf{b}^{\prime} q}^{\mathbf{a}}$ or in $d_{\mathbf{a}^{\prime \prime} \mathbf{t}^{\prime \prime} \mathbf{b}^{\prime \prime} q}^{\mathbf{b}}$ is bounded and independent of $\mathbb{F}_{q}$.

\section{$\S 5$. The integral bases from the Kronecker quiver}

Most results in this section can be found in [Z2] and [C] while others can be found in $[\mathrm{BK}]$.

\section{$\S 5.1$}

Let $\mathbb{F}_{q}$ be the finite field with $q$ elements and $Q$ be the Kronecker quiver with $I=\{1,2\}$ and $H=\left\{\rho_{1}, \rho_{2}\right\}$ such that $s\left(\rho_{1}\right)=s\left(\rho_{2}\right)=2$ and $t\left(\rho_{1}\right)=t\left(\rho_{2}\right)=1$. Let $\Lambda_{q}=\mathbb{F}_{q} Q$ be the path algebra. In this section we will use the subscript $q$ to indicate the dependence on $q$ since our computation will be performed in the category $\bmod \Lambda_{q}$. It is known that the structure of the preprojective and preinjective components of $\bmod \Lambda_{q}$ is the same as those of $\bmod k Q$ for $k$ being an algebraically closed field. However the regular components of $\bmod \Lambda_{q}$ are different from those of $\bmod k Q$. Recall that a module is called regular if all indecomposable direct summands are regular. By [R6], the full subcategory $\mathcal{R}$ of $\bmod \Lambda_{q}$ consisting of regular representations in $\bmod \Lambda_{q}$ is an abelian category. If $X$ is a simple object in $\mathcal{R}$, then $X$ is said to be a quasi-simple module in $\bmod \Lambda_{q}$.

The set of dimension vectors of indecomposable modules in $\bmod \Lambda$ is

$$
\Phi^{+}=\{(l+1, l),(m, m),(n, n+1) \mid l \geq 0, m \geq 1, n \geq 0\} .
$$


The dimension vectors $(n+1, n)$ and $(n, n+1)$ correspond to preprojective and preinjective indecomposable modules respectively and are real roots. For each real root $\alpha$, there is only one isoclass of indecomposable modules with dimension vector $\alpha$, which will be denoted by $V_{\alpha}$. Define a total order $<$ on $\Phi^{+}$by using the strong representation-directed property of the quiver $Q$,

$$
\begin{aligned}
(1,0)<\cdots<(m+1, m)<(m+ & 2, m+1)<\cdots<(k, k)<(k+1, k+1) \\
& <\cdots<(n+1, n+2)<(n, n+1)<\cdots<(0,1)
\end{aligned}
$$

so that there is no non-zero homomorphism from an indecomposable module of dimension vector $\alpha$ to an indecomposable module of dimension vector $\beta$ if $\beta<\alpha$. This property will be used frequently in the computation.

Any $\Lambda_{q}$-module is given by a quadruple $\left(V_{1}, V_{2} ; \sigma, \tau\right)$, where $V_{1}$ and $V_{2}$ are finite-dimensional vector spaces over $\mathbb{F}_{q}$, and $\sigma$ and $\tau$ are $\mathbb{F}_{q}$-linear maps from $V_{2}$ to $V_{1}$.

Proposition 5.1. The isomorphism classes of regular simple modules in $\bmod \Lambda_{q}$ are indexed by $\operatorname{spec}\left(\mathbb{F}_{q}[x]\right)$. That is, each regular quasi-simple module is isomorphic to $\left(V_{1}, V_{2} ; \sigma, \tau\right)$, where $V_{1}=V_{2}=\mathbb{F}_{q}[x] /(p(x))$ for an irreducible polynomial $p(x)$ in $\mathbb{F}_{q}[x], \sigma$ is the identity map and $\tau$ is given by multiplying by $x$, except $\left(\mathbb{F}_{q}, \mathbb{F}_{q} ; 0,1\right)$ which corresponds to the zero ideal.

\section{$\S 5.2$}

In this section, let $\mathcal{P}_{q}$ be the set of isomorphism classes of finite-dimensional $\Lambda_{q^{-}}$ modules, $\mathcal{H}_{q}$ be the Ringel-Hall algebra of $\Lambda_{q}$ over $\mathbb{Q}\left(v_{q}\right)$, where $v_{q}^{2}=q$, and $\mathcal{H}_{q}^{*}$ be the twisted form of $\mathcal{H}_{q}$. If $\mathbf{d} \in \mathbb{N} I$ is a dimension vector, we set in $\mathcal{H}_{q}$

$$
R_{\mathbf{d}}=\sum_{\substack{[M] \in \mathcal{P}_{q}, M \text { regular } \\ \underline{\operatorname{dim}} M=\mathbf{d}}} u_{[M]} .
$$

For an element $x=\sum_{[M] \in \mathcal{P}} c_{[M]} u_{[M]} \in \mathcal{H}_{q}$, we call $u_{[M]}$ a (non-zero) term of $x$ if $c_{[M]} \neq 0$. Furthermore,

$$
R(x)=\sum_{[M] \in \mathcal{P}_{q}, M \text { regular }} c_{[M]} u_{[M]}
$$

is called the regular part of $x$. According to our notation, we write $u_{\alpha}=u_{\left[V_{\alpha}\right]}$ for $\alpha=(n-1, n)$ or $(n, n+1)$ being real roots.

Let $\alpha_{1}=(1,0)$ and $\alpha_{2}=(0,1)$ be the simple roots. The orientation of $Q$ implies $\left\langle\alpha_{1}, \alpha_{2}\right\rangle=0$ and $\left\langle\alpha_{2}, \alpha_{1}\right\rangle=-2$. Thus for $\delta=(1,1)$ we have $\left\langle\delta, \alpha_{1}\right\rangle=-1$, $\left\langle\alpha_{1}, \delta\right\rangle=1,\left\langle\delta, \alpha_{2}\right\rangle=1$ and $\left\langle\alpha_{2}, \delta\right\rangle=-1$. 


\section{$\S 5.3$}

In this section, multiplication in $\mathcal{H}_{q}$ will be simply written as $x y$ instead of $x \circ y$. The following four lemmas can be verified by direct computation as in [Z2].

Lemma 5.2. Let $i$ and $j$ be positive integers. Then

$$
u_{(j-1, j)} u_{(i, i-1)}=R\left(u_{(j-1, j)} u_{(i, i-1)}\right)+q^{i+j-2} u_{(i, i-1)} u_{(j-1, j)} .
$$

\section{Lemma 5.3.}

$$
\begin{aligned}
R_{\delta} & =u_{(0,1)} u_{(1,0)}-u_{(1,0)} u_{(0,1)}, \\
u_{(n+1, n)} & =\frac{1}{q+1}\left(R_{\delta} u_{(n, n-1)}-q u_{(n, n-1)} R_{\delta}\right), \\
u_{(n, n+1)} & =\frac{1}{q+1}\left(u_{(n-1, n)} R_{\delta}-q R_{\delta} u_{(n-1, n)}\right) .
\end{aligned}
$$

Lemma 5.4. Let $i$ and $j$ be positive integers and $n=i+j-1$. Then

$$
R\left(u_{(j-1, j)} u_{(i, i-1)}\right)=R\left(u_{(n-1, n)} u_{(1,0)}\right)=R\left(u_{(0,1)} u_{(n, n-1)}\right) .
$$

Lemma 5.5 ([Z2, Theorem 4.2,4.3]). Let $m, n \geq 1$. Then

$$
\begin{aligned}
& u_{(m-1, m)} R_{n \delta}=\sum_{0 \leq i \leq n} \frac{q^{i}-q^{n+1}}{1-q} R_{i \delta} u_{(m+n-i-1, m+n-i)}, \\
& R_{n \delta} u_{(m, m-1)}=\sum_{0 \leq i \leq n} \frac{q^{i}-q^{n+1}}{1-q} u_{(m+n-i, m+n-i-1)} R_{i \delta} .
\end{aligned}
$$

\section{$\S 5.4$}

We will introduce a new set of elements in $\mathcal{H}_{q}^{*}$ to describe a basis that resembles a PBW basis for the universal enveloping algebra of a Lie algebra. We give here some quantum commutator relations in $\mathcal{H}_{q}$ and in $\mathcal{H}_{q}^{*}$. We define (cf. $\S 1.2$ )

$$
E_{(n+1, n)}=\left\langle u_{(n+1, n)}\right\rangle=v_{q}^{-2 n} u_{(n+1, n)}, \quad E_{(n, n+1)}=\left\langle u_{(n, n+1)}\right\rangle=v_{q}^{-2 n} u_{(n, n+1)} .
$$

We will call $E_{1}=E_{(1,0)}$ and $E_{2}=E_{(0,1)}$ the Chevalley generators. For $n \geq 1$, define in $\mathcal{H}^{*}$

$$
\tilde{E}_{n \delta}=E_{(n-1, n)} * E_{1}-v_{q}^{-2} E_{1} * E_{(n-1, n)} .
$$

Most of the computations below are known from [C] and/or [Z2].

Lemma 5.6 ([C, Lemma 3.5], [Z2]). $\tilde{E}_{n \delta}=v_{q}^{-3 n+1} R\left(u_{(n-1, n)} u_{(1,0)}\right)$. 
Proof. By taking $u_{1}=u_{(1,0)}$ we have

$$
\begin{aligned}
\tilde{E}_{n \delta} & =v_{q}^{-2(n-1)}\left(v_{q}^{\left\langle(n-1) \delta+\alpha_{2}, \alpha_{1}\right\rangle} u_{(n-1, n)} u_{1}-v_{q}^{-2} v_{q}^{\left\langle\alpha_{1},(n-1) \delta+\alpha_{2}\right\rangle} u_{1} u_{(n-1, n)}\right) \\
& =v_{q}^{-3 n+1}\left(u_{(n-1, n)} u_{1}-v_{q}^{2(n-1)} u_{1} u_{(n-1, n)}\right) \\
& =v_{q}^{-3 n+1} R\left(u_{(n-1, n)} u_{1}\right) \quad \text { by Lemma } 5.2 .
\end{aligned}
$$

Lemma 5.7 ([C, Prop. 4.2]). There exist $a_{h}^{(r)}(v), b_{h}^{(r)}(v) \in \mathbb{Z}\left[v, v^{-1}\right]$ for $r \in \mathbb{N} \backslash\{0\}$ and $h \in\{0,1, \ldots,\lfloor r / 2\rfloor\}$ such that for all $n>m$ in $\mathbb{N}$,

$$
\begin{aligned}
& E_{(n+1, n)} * E_{(m+1, m)}=\sum_{h=0}^{\lfloor(n-m) / 2\rfloor} a_{h}^{(n-m)}\left(v_{q}\right) E_{(m+h+1, m+h)} * E_{(n-h+1, n-h)}, \\
& E_{(m, m+1)} * E_{(n, n+1)}=\sum_{h=0}^{\lfloor(n-m) / 2\rfloor} b_{h}^{(n-m)}\left(v_{q}\right) E_{(n-h+1, n-h)} * E_{(m+h, m+h+1)} .
\end{aligned}
$$

For $k \geq 0$, we inductively define

$$
E_{0 \delta}=1, \quad E_{k \delta}=\frac{1}{[k]} \sum_{s=1}^{k} v_{q}^{s-k} \tilde{E}_{s \delta} * E_{(k-s) \delta} .
$$

Lemma 5.8. We have $E_{k \delta}=v_{q}^{-2 k} R_{k \delta}$.

Proof. If $k=1$, then $E_{\delta}=\tilde{E}_{\delta}=v_{q}^{-2} R_{\delta}$. Assume that the assertion is true for all $t<k$. Then by Lemma 5.6, and [Z2, Lem. 3.7, Thm. 4.1, Lem. 4.7],

$$
\begin{aligned}
E_{k \delta} & =\frac{1}{[k]} \sum_{s=1}^{k} v_{q}^{s-k} v_{q}^{-3 s+1} R\left(u_{(s-1, s)} u_{1}\right) * v_{q}^{-2(k-s)} R_{(k-s) \delta} \\
& =\frac{1}{[k]} \sum_{s=1}^{k} v_{q}^{-3 k+1} R\left(u_{(s-1, s)} u_{1}\right) * R_{(k-s) \delta} \\
& =\frac{1}{[k]} \sum_{s=1}^{k} v_{q}^{-3 k+1} a_{s}\left(R_{\delta}, R_{2 \delta}, \ldots, R_{s \delta}\right) * R_{(k-s) \delta} \\
& =\frac{v_{q}^{-3 k+1}}{[k]} \frac{1-q^{k}}{1-q} R_{k \delta}=v_{q}^{-2 k} R_{k \delta} .
\end{aligned}
$$

Here $a_{s}\left(R_{\delta}, R_{2 \delta}, \ldots, R_{s \delta}\right)$ is as in [Z2, p. 105].

Lemma 5.9. For $m, n \in \mathbb{N}$ we have in $\mathcal{H}_{q}^{*}$

$$
\begin{aligned}
& E_{n \delta} * E_{(m+1, m)}=\sum_{k=0}^{n}[n+1-k] E_{(m+n+1-k, m+n-k)} * E_{k \delta}, \\
& E_{(m, m+1)} * E_{n \delta}=\sum_{k=0}^{n}[n+1-k] E_{k \delta} * E_{(m+n-k, m+n-k+1)} .
\end{aligned}
$$


Proof. Again we will only verify the first equality. By Lemma 5.8, we have

$$
\begin{aligned}
E_{n \delta} * E_{(m+1, m)}=v_{q}^{-2 n} R_{n \delta} * v_{q}^{-2 m} u_{(m+1, m)}=v_{q}^{-2(n+m)} v_{q}^{-n} R_{n \delta} u_{(m+1, m)} \\
=v_{q}^{-3 n-2 m} \sum_{k=0}^{n} \frac{q^{k}-q^{n+1}}{1-q} u_{(m+n-k+1, m+n-k)} R_{k \delta} \quad(\text { by Lemma 5.5) } \\
=\sum_{k=0}^{n} \frac{v_{q}^{-3 n-2 m}}{1-v_{q}^{2}}\left(v_{q}^{2 k}-v_{q}^{2 n+2}\right) v_{q}^{2 k} v_{q}^{-k} v_{q}^{2(n+m-k)} E_{(m+n-k+1, m+n-k)} * E_{k \delta} \\
=\sum_{k=0}^{n}[n+1-k] E_{(m+n-k+1, m+n-k)} * E_{k \delta} .
\end{aligned}
$$

$\S 5.5$

Let $\mathcal{L}_{q}$ be the $\mathcal{Z}_{v_{q}}=\mathbb{Z}\left[v_{q}, v_{q}^{-1}\right]$-subalgebra of $\mathcal{H}_{q}^{*}$ generated by the set

$$
\left\{E_{(m+1, m)}^{(* s)}, E_{k \delta}, E_{(n, n+1)}^{(* t)} \mid m, n \geq 0, s, t, k \geq 1\right\} .
$$

Recall from $\S 1.4$ that the integral $\mathcal{Z}_{v_{q}}$-form $\mathcal{C}_{\mathcal{Z}_{v_{q}}}^{*}$ is generated by $E_{(1,0)}^{(* s)}$ and $E_{(0,1)}^{(* s)}$. Thus $\mathcal{C}_{\mathcal{Z}_{v_{q}}}^{*} \subseteq \mathcal{L}_{q}$.

On the other hand, by $\S 4.2$, we get $E_{(m+1, m)}^{\left(* s_{m}\right)}, E_{(n, n+1)}^{\left(* t_{n}\right)} \in \mathcal{C}_{\mathcal{Z}_{v_{q}}}^{*}$. Because $E_{n \delta}$ is just $\widetilde{P}_{n, 1}$ in $[\mathrm{BCP}]$, this implies $E_{k \delta}^{r_{k}} \in \mathcal{C}_{\mathcal{Z}_{v_{q}}}^{*}$. Thus $\mathcal{L}_{q} \subseteq \mathcal{C}_{\mathcal{Z}_{v_{q}}}^{*}$. Therefore $\mathcal{C}_{\mathcal{Z}_{v_{q}}}^{*}=\mathcal{L}_{q}$.

Now we have an integral $\mathcal{Z}_{v_{q}}$-basis of $\mathcal{L}_{q}$ consisting of the monomials

$$
\left\{\prod_{m \geq 0} E_{(m+1, m)}^{\left(* s_{m}\right)} \prod_{k \geq 1} E_{k \delta}^{* r_{k}} \prod_{n \geq 0} E_{(n, n+1)}^{\left(* t_{n}\right)} \mid s_{m}, t_{n}, r_{k} \geq 0\right\}
$$

with the product taken with respect to the order given in $\S 5.1$ and there are only finitely many non-zero $s_{m}, t_{n}$, and $r_{k}$ in each monomial. This follows easily from the facts: (1) those monomials are linearly independent over $\mathcal{Z}_{v_{q}}$ (even over $\mathbb{Q}\left(v_{q}\right)$ ) by the definition of Ringel-Hall algebras; (2) because there exist Hall polynomials in the Kronecker quiver by [Z2], it follows from the lemmas in $\S 5.4$ that the $\mathcal{Z}_{v_{q}}$ span of the monomials above is closed under multiplication in $\mathcal{H}_{q}^{*}$ and that $\mathcal{L}_{q}$ contains all monomials we defined above.

Remark. The formulae in the lemmas are unchanged when we vary $q$. The lemmas can be stated in $\prod_{q} \mathcal{H}_{q}^{*}$ with $v_{q}$ replaced by $v=\left(v_{q}\right)$ (as a variable) in $\prod_{q} \mathcal{Z}_{v_{q}}$ and with $E_{(*, *)}$ replaced by $E_{(*, *)}=\left(E_{(*, *), q}\right)$. We then define $\mathcal{L}$ as the $\mathcal{Z}=\mathbb{Z}\left[v, v^{-1}\right]$-algebra with a $\mathcal{Z}$-basis consisting of the monomials described above.

As remarked in $\S 1.4$, Lusztig's integral $\mathcal{Z}$-form $\mathcal{C}_{\mathcal{Z}}^{*}$, which is called the generic composition algebra, can be viewed as a $\mathcal{Z}$-subalgebra of $\prod_{q} \mathcal{H}_{q}^{*}$ by the RingelGreen theorem (see $[\mathrm{G}],[\mathrm{R} 1, \mathrm{R} 7]$ ). Using this identification, we can view $\mathcal{C}_{\mathcal{Z}}^{*}=\mathcal{L}$. 
Let $\mathbf{P}(n)$ be the set of all partitions of $n$. Recall that there are no nontrivial extensions between homogeneous regular representations. For any $w=$ $\left(w_{1}, \ldots, w_{m}\right) \in \mathbf{P}(n)$, we define

$$
E_{w \delta}=E_{w_{1} \delta} * \cdots * E_{w_{m} \delta}
$$

By Lemmas 5.5, 5.8, and the definition of $\langle M\rangle$, we get $\mathcal{L} \subseteq \mathcal{C}_{\mathcal{Z}}^{*}$. Then we have

Proposition 5.10. The set

$$
\left\{\langle P\rangle * E_{w \delta} *\langle I\rangle \mid P \in \mathcal{P}_{\text {prep }}, w \in \mathbf{P}(n), I \in \mathcal{P}_{\text {prei }}, n \in \mathbb{N}\right\}
$$

is a $\mathcal{Z}$-basis of $\mathcal{C}_{\mathcal{Z}}^{*}$. Here $\mathcal{P}_{\text {prep }}$ and $\mathcal{P}_{\text {prei }}$ are, respectively, the isoclasses of preprojective and preinjective modules in $\mathcal{P}_{q}$.

Remarks. (1) It has been proved by Zhang in [Z2] that the basis in Proposition 5.10 is a $\mathbb{Q}(v)$-basis of $U^{+}$. Chen $[\mathrm{C}]$ improved this and showed that this set is a $\mathcal{Z}$-basis of $U_{\mathcal{Z}}^{+}$.

(2) It is not difficult to see that elements in $\left\{E_{n \delta} \mid n \in \mathbb{N}\right\}$ constructed here correspond to the root vectors of $U_{q}\left(\hat{s l_{2}}\right)$ constructed by Damiani in [Da] and by Beck in $[\mathrm{Be}]$.

(3) It can be proved in an easier way that the set in Proposition 5.10 is an integral basis of $\mathcal{C}_{\mathcal{A}}^{*}$ over $\mathcal{A}=\mathbb{Q}\left[v, v^{-1}\right]$. (See the proof of Proposition 6.2 below.)

We end this section with a lemma which will be used in Section 9. For any $n>$ $m \geq 0, P_{(n, m)}$ (resp. $\left.I_{(m, n)}\right)$ denotes a preprojective (resp. preinjective) module with $\underline{\operatorname{dim}} P_{(n, m)}=(n, m)$ (resp. $\left.\underline{\operatorname{dim}} I_{(m, n)}=(m, n)\right)$. In the following formulae, the summations are over all nonzero preprojective and preinjective modules with the indicated dimension vectors. The formulas can first be stated for representations over $\mathbb{F}_{q}$. By using the argument in $\S 1.4$, we will state them in $\prod_{q} \mathcal{H}_{q}^{*}$ with $v$ as a variable.

Lemma 5.11. In the following formulas all $P$ and $I$ are non-zero.

$$
\begin{aligned}
& \text { (i) } E_{2}^{(* n)} * E_{1}^{(*(n+1))}=E_{(n+1, n)}+\sum_{1 \leq l \leq n} v^{-l-1} E_{(n-l+1, n-l)} * E_{l \delta} \\
& +\sum_{\substack{0 \leq l \leq n-1 \\
p \geq 1, s \geq 0, t \geq 0 \\
s+t+l+(p-1)=n}} v^{-\operatorname{dim} \text { End } P-\operatorname{dim} \text { End } I} v^{-p(l+t)-(s+l)(p-1)}\left\langle P_{(s+p, s)}\right\rangle * E_{l \delta} *\left\langle I_{(t, t+p-1)}\right\rangle ;
\end{aligned}
$$


(ii) $\quad E_{2}^{(*(n+1))} * E_{1}^{(* n)}=E_{(n, n+1)}+\sum_{1 \leq l \leq n} v^{-l-1} E_{l \delta} * E_{(n-l, n-l+1)}$

$+\sum_{\substack{0 \leq l \leq n-1 \\ p \geq 1, s \geq 0, t \geq 0 \\ s+t+l+(p-1)=n}} v^{-\operatorname{dim} \operatorname{End} P-\operatorname{dim} \operatorname{End} I} v^{-p(l+s)+(t+l)(p-1)}\left\langle P_{(s+p-1, s)}\right\rangle * E_{l \delta} *\left\langle I_{(t, t+p)}\right\rangle ;$

(iii) $\quad E_{2}^{(* n)} * E_{1}^{(* n)}=E_{n \delta}$

$+\sum_{\substack{0 \leq l \leq n-1, p \geq 1 \\ s \geq 0, t \geq 0, s+t+l+p=n}} v^{-\operatorname{dim} \operatorname{End} P-\operatorname{dim} \operatorname{End} I} v^{-p(s+2 l+t)}\left\langle P_{(s+p, s)}\right\rangle * E_{l \delta} *\left\langle I_{(t, t+p)}\right\rangle$.

Proof. We work with representations over $\mathbb{F}_{q}$ and verify (i) only. The others can be verified in a similar way. We have the following relation in $\mathcal{H}$ (see [R3]):

$$
\begin{aligned}
u_{2}^{n} u_{1}^{n+1}=\psi_{n}(q) \psi_{n+1}(q)\left(u_{(n+1, n)}+\right. & \sum_{1 \leq l \leq n} u_{(n-l+1, n-l)} R_{l \delta} \\
& \left.+\sum_{\substack{0 \leq l \leq n-1, p \geq 1 \\
s \geq 0, t \geq 0, s+t+l+(p-1)=n}} u_{[P]} R_{l \delta} u_{[I]}\right)
\end{aligned}
$$

where $P$ is a non-zero preprojective module with $\underline{\operatorname{dim}} P=(s+p, s)$ and $I$ is a nonzero preinjective module with $\underline{\operatorname{dim}} I=(t, t+p-1)$. Then by a routine calculation using the relation in $\S 5.3$, we obtain (i).

\section{$\S 6$. Integral bases for the generic composition algebras}

\section{$\S 6.1$}

For a connected tame quiver $Q$ without oriented cycles with path algebra $\Lambda=\mathbb{F}_{q} Q$, let $e$ be an extending vertex of $Q$. Let $P=P(e)$ be the projective cover of the simple module $S_{e}$ corresponding to the vertex $e$. Setting $\mathfrak{p}=\underline{\operatorname{dim}} P(e)$, one has $\langle\mathfrak{p}, \mathfrak{p}\rangle=1=\langle\mathfrak{p}, \delta\rangle$ and there exists a unique indecomposable preprojective module $L$ with $\underline{\operatorname{dim}} L=\mathfrak{p}+\delta$. Moreover $\operatorname{Hom}_{\Lambda}(L, P)=0$ and $\operatorname{Ext}_{\Lambda}(L, P)=0$. Recall that a pair $(M, N)$ of indecomposable $\Lambda$-modules is called an exceptional pair if $\operatorname{Ext}(M, M)=\operatorname{Ext}(N, N)=0$ and $\operatorname{Ext}(N, M)=\operatorname{Hom}(N, M)=0$. Thus $(P, L)$ is an exceptional pair.

Let $\mathfrak{C}(P, L)$ be the smallest full subcategory of $\bmod \Lambda$ which contains $P$ and $L$ and is closed under taking extensions, kernels of epimorphisms, and cokernels of monomorphisms in the category of $\Lambda$-modules. Also we have $\operatorname{dim}_{\mathbb{F}_{q}} \operatorname{Hom}_{\Lambda}(P, L)=2$. By $[\mathrm{CB}], \mathfrak{C}(P, L)$ is equivalent to the category of $\mathbb{F}_{q} K$-modules, where $K$ is the Kronecker quiver with two arrows from vertex 2 to 1 . In this case, $L$ corresponds to the projective cover of the simple module $\theta_{2}$ and $P$ corresponds to the projective 
cover of the simple module $\theta_{1}$. This induces an exact embedding $F: \bmod \mathbb{F}_{q} K \hookrightarrow$ $\bmod \Lambda$. We note here that the embedding functor $F$ is essentially independent of the field $\mathbb{F}_{q}$. This gives rise to an injective homomorphism of algebras, still denoted by $F: \mathcal{H}_{q}^{*}(K) \hookrightarrow \mathcal{H}_{q}^{*}(\Lambda)$.

In $\mathcal{H}_{q}^{*}(K)$ we have defined, in $\S 5.8$, the elements $E_{m \delta_{K}}$ for $m \geq 1$ for the corresponding imaginary root $\delta_{K}$. The images $E_{m \delta}=F\left(E_{m \delta_{K}}\right)$ in $\mathcal{H}_{q}^{*}(Q)$ will play a significant role in the construction of PBW bases and canonical bases in the affine cases. Since $E_{m \delta_{K}} \in \mathcal{C}_{q}^{*}(K)$, and $\langle L\rangle,\langle P\rangle \in \mathcal{C}_{q}^{*}(\Lambda)$, it follows that $E_{m \delta}$ is in $\mathcal{C}_{q}^{*}(\Lambda)$. Therefore the genericalization process of $\S 1.4$ will induce an embedding of algebras $F: \mathcal{C}^{*}(K)_{\mathcal{Z}} \rightarrow \mathcal{C}^{*}(\Lambda)_{\mathcal{Z}}$. Let $\mathcal{K}$ be the subalgebra of $\mathcal{C}^{*}(\Lambda)_{\mathcal{Z}}$ generated by $E_{m \delta}$ for $m \in \mathbb{N}$. It is a polynomial ring on infinitely many variables $\left\{E_{m \delta} \mid m \geq 1\right\}$, and its integral form is the polynomial ring on the variables $\left\{E_{m \delta} \mid m \geq 1\right\}$ over $\mathcal{Z}$ although the expressions of $E_{m \delta}$ in terms of linear combinations of equivalence classes of modules of $\Lambda$ will vary as $q$ changes. In a certain sense, these elements will collect the contributions of the homogeneous regular modules to the composition algebras.

\section{$\S 6.2$}

Let $\mathcal{T}_{1}, \ldots, \mathcal{T}_{s}$ be all non-homogeneous tubes in $\bmod \Lambda$ (in fact, $s \leq 3$ ). For each $\mathcal{T}_{i}$, let $r_{i}=r\left(\mathcal{T}_{i}\right)$ be the period of $\mathcal{T}_{i}$, i.e., the number of quasi-simple modules in $\mathcal{T}_{i}$. Then $r_{i}>1$. Let $\mathfrak{g}$ be the Kac-Moody Lie algebra corresponding to the tame quiver $Q$. The multiplicity of a root $\alpha$ is the dimension of the root space $\mathfrak{g}_{\alpha}$. The following is well-known (for example see $[\mathrm{CB}]$ ):

Lemma 6.1. We have the equality $\sum_{i=1}^{s}\left(r_{i}-1\right)=|I|-2$ and the multiplicity of each imaginary root $m \delta$ is $|I|-1$, where $|I|$ is the number of vertices of $Q$.

\section{$\S 6.3$}

Each non-homogeneous tube $\mathcal{T}_{i}$ is a full subcategory of $\bmod \Lambda$, closed under extensions and equivalent to the full subcategory of nilpotent modules of the cyclic quiver of the same period. In Section 3 , the composition algebra $\mathcal{C}_{q}^{*}\left(\mathcal{T}_{i}\right)$ of $\mathcal{T}_{i}$ and its generic integral form $\mathcal{C}_{\mathcal{Z}}^{*}\left(\mathcal{T}_{i}\right)$ have been constructed. For each $\mathcal{T}_{i}$, let $\Pi_{i}^{a}$ be the set of aperiodic $r_{i}$-tuples of partitions such that $M_{i}\left(\pi_{i}\right)$ is an aperiodic module in $\mathcal{T}_{i}$ for any $\pi_{i} \in \Pi_{i}^{a}$. We have constructed in $\S 3.3$ the element

$$
E_{\pi_{i}}=\left\langle M_{i}\left(\pi_{i}\right)\right\rangle+\sum_{\lambda_{i} \in \Pi_{i} \backslash \Pi_{i}^{a}, \lambda_{i} \prec \pi_{i}} \eta_{\lambda_{i}}^{\pi_{i}}\left\langle M_{i}\left(\lambda_{i}\right)\right\rangle
$$

in $\mathcal{C}^{*}\left(\mathcal{T}_{i}\right)_{\mathcal{Z}}$. Then $\left\{E_{\pi_{i}} \mid \pi_{i} \in \Pi_{i}^{a}\right\}$ is a $\mathcal{Z}$-basis of $\mathcal{C}^{*}\left(\mathcal{T}_{i}\right)_{\mathcal{Z}}$. 
There is a natural embedding of $\mathcal{H}_{q}\left(\mathcal{T}_{i}\right)$ into $\mathcal{H}_{q}(\Lambda)$ from the embedding of the category $\mathcal{T}_{i}$ in $\bmod \Lambda$. Since the Euler form $\langle-,-\rangle$ defined in $\S 1.2$ depends on the homomorphism and extension spaces only, this embedding also gives an algebra embedding $\mathcal{H}_{q}^{*}\left(\mathcal{T}_{i}\right)$ into $\mathcal{H}_{q}^{*}(\Lambda)$ for each finite field $\mathbb{F}_{q}$. Since all quasisimple modules in $\mathcal{T}_{i}$ are in $\mathcal{C}_{q}^{*}(\Lambda)$, we have $\mathcal{C}_{q}^{*}\left(\mathcal{T}_{i}\right) \subseteq \mathcal{C}_{q}^{*}(\Lambda)$ for all $q$. This induces an embedding of $\mathcal{C}^{*}\left(\mathcal{T}_{i}\right)_{\mathcal{Z}}$ into $\mathcal{C}^{*}(\Lambda)_{\mathcal{Z}}$. Thus elements in $\mathcal{C}^{*}\left(\mathcal{T}_{i}\right)_{\mathcal{Z}}$ will be denoted by the same notations as their images in $\mathcal{C}^{*}(\Lambda)_{\mathcal{Z}}$.

For the tame quiver $Q$, let $\mathcal{M}$ be the set of quadruples $\mathbf{c}=\left(\mathbf{a}_{\mathbf{c}}, \mathbf{b}_{\mathbf{c}}, \pi_{\mathbf{c}}, w_{\mathbf{c}}\right)$ such that $\mathbf{a}_{\mathbf{c}} \in \mathbb{N}_{f}^{P r e p}, \mathbf{b}_{\mathbf{c}} \in \mathbb{N}_{f}^{P r e i}, \pi_{\mathbf{c}}=\left(\pi_{1 \mathbf{c}}, \ldots, \pi_{s \mathbf{c}}\right) \in \Pi_{1}^{a} \times \cdots \times \Pi_{s}^{a}$, and $w_{\mathbf{c}}=\left(w_{1} \geq \cdots \geq w_{t}\right)$ is a partition.

Then for each $\mathbf{c} \in \mathcal{M}$ we define

$$
E^{\mathbf{c}}=\left\langle M\left(\mathbf{a}_{\mathbf{c}}\right)\right\rangle * E_{\pi_{2 \mathbf{c}}} * \cdots * E_{\pi_{s \mathbf{c}}} * E_{w_{\mathbf{c}} \delta} *\left\langle M\left(\mathbf{b}_{\mathbf{c}}\right)\right\rangle,
$$

where $\left\langle M\left(\mathbf{a}_{\mathbf{c}}\right)\right\rangle$ and $\left\langle M\left(\mathbf{b}_{\mathbf{c}}\right)\right\rangle$ are defined in Lemmas 4.4 and 4.2 respectively, $E_{\pi_{i \mathbf{c}}}$ is defined above and $E_{w_{\mathbf{c}} \delta}$ is defined in $\S 6.1$ and Lemma 5.8. Obviously, $\left\{E^{\mathbf{c}} \mid \mathbf{c} \in \mathcal{M}\right\}$ lie in $\mathcal{C}^{*}(\Lambda)$, in fact in $\mathcal{C}^{*}(\Lambda)_{\mathcal{Z}}$, and are linearly independent over $\mathbb{Q}(v)$.

Proposition 6.2. The set $\left\{E^{\mathbf{c}} \mid \mathbf{c} \in \mathcal{M}\right\}$ is a $\mathbb{Q}(v)$-basis of $\mathcal{C}^{*}(\Lambda)_{\mathbb{Q}(v)}$.

The proof of Proposition 6.2 will be given in $\S 6.4$ after some preparations.

Lemma 6.3. In $\mathcal{C}^{*}(\Lambda)_{\mathcal{Z}}$,

$$
\tilde{E}_{n \delta}=\sum_{\substack{m_{1} \leq \cdots \leq m_{s} \\ m_{1}+\cdots+m_{s}=n}} b_{m_{1}, \ldots, m_{s}} E_{m_{1} \delta} * \cdots * E_{m_{s} \delta}, \quad \text { where } b_{m_{1}, \ldots, m_{s}} \in \mathcal{Z} \text {. }
$$

Proof. By the relation

$$
E_{0 \delta}=1, \quad E_{k \delta}=\frac{1}{[k]} \sum_{s=1}^{k} v^{s-k} \tilde{E}_{s \delta} * E_{(k-s) \delta},
$$

we can solve the equation recursively to get the relation in the lemma.

It is known from Ringel's work $[\mathrm{R} 2]$ that the Lie subalgebra $\mathfrak{n}^{+} \subseteq$ $\mathcal{C}^{*}(\Lambda)_{\mathbb{Q}\left[v, v^{-1}\right]} /(v-1) \mathcal{C}^{*}(\Lambda)_{\mathbb{Q}\left[v, v^{-1}\right]}$ generated by $u_{\left[S_{i}\right]}(i \in I)$ over $\mathbb{Q}$ is the positive part of the corresponding affine Kac-Moody Lie algebra over $\mathbb{Q}$, and $\mathcal{C}^{*}(\Lambda)_{\mathbb{Q}\left[v, v^{-1}\right]} /(v-1) \mathcal{C}^{*}(\Lambda)_{\mathbb{Q}\left[v, v^{-1}\right]}$ is the universal enveloping algebra of $\mathfrak{n}^{+}$.

For each non-homogeneous tube $\mathcal{T}_{i}$ of rank $r_{i}$, we write $S_{j}[l]_{i}$ for the indecomposable module $S_{j}[l]$ in the tube $\mathcal{T}_{i}$. Let $\Psi: \mathcal{C}^{*}(\Lambda)_{\mathcal{Z}} \rightarrow \mathcal{C}^{*}(\Lambda)_{\mathcal{Z}} /(v-1) \mathcal{C}^{*}(\Lambda)_{\mathcal{Z}}$ be the canonical projection. Then one of the main results in [FMV] is the following; its proof relies on Lemma 6.1. 
Proposition 6.4. The vectors $\Psi\left(u_{[M(\alpha)]}\right)$ for $\alpha \in \Phi_{\text {Prep }}^{+} ; \Psi\left(u_{\left[S_{j}[l]_{i}\right]}\right)$ for $\operatorname{dim} S_{j}[l]_{i}$ $=\alpha$ being a real root, $i=1, \ldots, s ; \Psi\left(u_{\left[S_{j}[l]_{i}\right]}-u_{\left[S_{j+1}[l]_{i}\right]}\right)$ for $\underline{\operatorname{dim}} S_{j}[l]_{i}=m \delta$ being an imaginary root and $1 \leq j \leq r_{i}-1, i=1, \ldots, s ; \Psi\left(\tilde{E}_{n \delta}\right), n \geq 1$; and $\Psi\left(u_{[M(\beta)]}\right)$ for $\beta \in \Phi_{\text {Prei }}^{+}$, form a $\mathbb{Z}$-basis of $\mathfrak{n}^{+}$.

Note that it is easy to see that all vectors in Proposition 6.4 belong to the Lie algebra $\mathfrak{n}^{+}$, and that they are linearly independent over $\mathbb{Q}$. For example, $\Psi\left(\tilde{E}_{n \delta}\right)$ is in $\mathfrak{n}^{+}$for all $n \geq 1$. Then by Lemma 6.1 , one can prove that those vectors give rise to a $\mathbb{Z}$-basis of $\mathfrak{n}^{+}$.

\section{$\S 6.4$. Proof of Proposition 6.2}

By the definition, $\left\{E^{\mathbf{c}} \mid \mathbf{c} \in \mathcal{M}\right\}$ is a linearly independent set over $\mathbb{Q}(v)$. For any dimension vector $w \in \mathbb{N} I$, let $V_{w}$ be the $\mathbb{Q}(v)$-subspace spanned by those $E^{\mathbf{c}}$, $\mathbf{c} \in \mathcal{M}$, such that $E^{\mathbf{c}} \in \mathcal{C}^{*}(\Lambda)_{w}$. It is well-known from Lusztig's work [L1] that

$$
\operatorname{dim}_{\mathbb{Q}(v)} \mathcal{C}^{*}(\Lambda)_{w}=\operatorname{dim}_{\mathbb{Q}}\left(\mathcal{C}^{*}(\Lambda)_{\mathbb{Q}\left[v, v^{-1}\right]} /(v-1) \mathcal{C}^{*}(\Lambda)_{\mathbb{Q}\left[v, v^{-1}\right]}\right)_{w}
$$

and the monomials in a fixed order in the basis elements of $\mathfrak{n}^{+}$in Proposition 6.4 form a PBW basis of $\mathcal{C}^{*}(\Lambda)_{\mathbb{Q}\left[v, v^{-1}\right]} /(v-1) \mathcal{C}^{*}(\Lambda)_{\mathbb{Q}\left[v, v^{-1}\right]}$ over $\mathbb{Q}$. However, Lemmas 3.3 and 6.3 imply that the PBW basis elements can be obtained by applying $\Psi$ on $\left\{E^{\mathbf{c}} \mid \mathbf{c} \in \mathcal{M}\right\}$. Therefore $\operatorname{dim}_{\mathbb{Q}(v)} V_{w} \geq \operatorname{dim}_{\mathbb{Q}(v)} \mathcal{C}^{*}(\Lambda)_{w}$. Hence $\left\{E^{\mathbf{c}} \mid \mathbf{c} \in \mathcal{M}\right\}$ is a $\mathbb{Q}(v)$-basis of $\mathcal{C}^{*}(\Lambda)$.

As a consequence, the multiplication map

$$
\varphi: \mathcal{C}^{*}(\operatorname{Prep}) \otimes_{\mathbb{Q}(v)} \mathcal{C}^{*}\left(\mathcal{T}_{1}\right) \otimes_{\mathbb{Q}(v)} \cdots \otimes_{\mathbb{Q}(v)} \mathcal{C}^{*}\left(\mathcal{T}_{s}\right) \otimes_{\mathbb{Q}(v)} \mathcal{K} \otimes_{\mathbb{Q}(v)} \mathcal{C}^{*}(\operatorname{Prei}) \rightarrow \mathcal{C}^{*}(\Lambda)
$$

is an isomorphism of $\mathbb{Q}(v)$-vector spaces.

\section{$\S 6.5$}

We may consider the ring $\mathcal{A}=\mathbb{Q}\left[v, v^{-1}\right]$ and denote by $\mathcal{C}^{*}(\Lambda)_{\mathcal{A}}$ the $\mathcal{A}$-subalgebra of the generic composition algebra $\mathcal{C}^{*}(\Lambda)_{\mathcal{A}} \subseteq \prod_{q} \mathcal{H}_{q}^{*}(\Lambda)$ generated by $u_{i}^{(* m)}=$ $u_{i}^{* m} /[m] !(i \in I)$.

Proposition 6.5. The set $\left\{E^{\mathbf{c}} \mid \mathbf{c} \in \mathcal{M}\right\}$ is an $\mathcal{A}$-basis of $\mathcal{C}^{*}(\Lambda)_{\mathcal{A}}$.

Proof. For any monomial $\mathfrak{m}$ in the divided powers of $u_{\left[S_{i}\right]}(i \in I)$, Proposition 6.2 implies

$$
\mathfrak{m}=\sum_{\mathbf{c} \in \mathcal{M}} f_{\mathfrak{m}, \mathbf{c}}(v) E^{\mathbf{c}} \in \mathcal{C}^{*}(\Lambda)
$$


where $f_{\mathfrak{m}, \mathbf{c}}(v) \in \mathbb{Q}(v)$ and the summation is finite. Note that $E_{\pi_{i \mathbf{c}}}$ in the definition of $E^{\mathbf{c}}$ has the form (cf. $\S 3.3$ )

$$
E_{\pi}=\langle M(\pi)\rangle+\sum_{\lambda \in \Pi_{\alpha} \backslash \Pi_{\alpha}^{a}, \lambda \prec \pi} \eta_{\lambda}^{\pi}\langle M(\lambda)\rangle
$$

with $\eta_{\lambda}^{\pi} \in \mathcal{Z}$. The formula $\mathfrak{m}=\sum_{\mathbf{c} \in \mathcal{M}} f_{\mathfrak{m}, \mathbf{c}}\left(v_{q}\right) E^{\mathbf{c}}$ still holds for each $q$. Thus, by Lemma 1.2, for each $\mathbf{c} \in \mathcal{M}$, there exists $N(\mathbf{c}) \in \mathbb{N}$ such that $(\sqrt{q})^{N(\mathbf{c})} f_{\mathfrak{m}, \mathbf{c}}(\sqrt{q}) \in \mathbb{Z}$ for all $q$. It is easily seen that $v^{N(\mathbf{c})} f_{\mathfrak{m}, \mathbf{c}}(v)$ is a polynomial in $\mathbb{Q}[v]$. Therefore $f_{\mathfrak{m}, \mathbf{c}}(v) \in \mathbb{Q}\left[v, v^{-1}\right]$.

Corollary 6.6. The multiplication map

$$
\varphi: \mathcal{C}^{*}(\operatorname{Prep})_{\mathcal{A}} \otimes_{\mathcal{A}} \mathcal{C}^{*}\left(\mathcal{T}_{1}\right)_{\mathcal{A}} \otimes_{\mathcal{A}} \cdots \otimes_{\mathcal{A}} \mathcal{C}^{*}\left(\mathcal{T}_{s}\right)_{\mathcal{A}} \otimes_{\mathcal{A}} \mathcal{K}_{\mathcal{A}} \otimes_{\mathcal{A}} \mathcal{C}^{*}(\text { Prei })_{\mathcal{A}} \rightarrow \mathcal{C}^{*}(\Lambda)_{\mathcal{A}}
$$

is an isomorphism of $\mathcal{A}$-modules.

\section{$\S 7$. A bar-invariant basis of $\mathcal{C}^{*}(\Lambda)_{\mathcal{A}}$}

\section{$\S 7.1$}

In this section, we continue to use the settings of Section 6 . The first part of this section is devoted to finding a monomial basis and a triangular relation with the PBW basis $\left\{E^{\mathbf{c}} \mid \mathbf{c} \in \mathcal{M}\right\}$.

We first define the variety

$$
\mathcal{O}_{\mathbf{c}}=\mathcal{O}_{M\left(\mathbf{a}_{\mathbf{c}}\right)} \star \mathcal{O}_{M_{\pi_{1 \mathbf{c}}}} \star \cdots \star \mathcal{O}_{M_{\pi_{s \mathbf{c}}}} \star \mathcal{N}_{w_{\mathbf{c}} \delta} \star \mathcal{O}_{M\left(\mathbf{b}_{\mathbf{c}}\right)}
$$

for any $\mathbf{c} \in \mathcal{M}$, where $\mathcal{N}_{w_{\mathbf{c}} \delta}=\mathcal{N}_{w_{1} \delta} \star \cdots \star \mathcal{N}_{w_{t} \delta}$ if $w_{\mathbf{c}}=\left(w_{1}, \ldots, w_{t}\right)$ and each $\mathcal{N}_{w_{i} \delta}$ is the union of orbits of regular modules of $\mathfrak{C}(P, L)$ with dimension vector $w_{i} \delta$. Since all homogeneous regular modules of dimension vector $w_{i} \delta$ of the affine quiver are in $\mathfrak{C}(P, L)$, by an argument similar to [R8, Cor.], $\mathcal{N}_{w_{i} \delta}$ is an irreducible variety.

Then by Proposition 6.5, Lemma 4.6 can be rewritten as follows:

Lemma 7.1. For any $\mathbf{a} \in \mathbb{N}_{f}^{\text {Prep }}$ and $\mathbf{b} \in \mathbb{N}_{f}^{\text {Prei }}$, in $\mathcal{C}^{*}(\Lambda)_{\mathcal{A}}$ we have

$$
\begin{aligned}
& \mathfrak{m}_{\mathbf{a}}=\langle M(\mathbf{a})\rangle+\sum_{\operatorname{dim} \mathcal{O}_{\mathbf{c}}<\operatorname{dim} \mathcal{O}_{\mathbf{a}}} f_{\mathbf{c}}^{\mathbf{a}} E^{\mathbf{c}}, \\
& \mathfrak{m}_{\mathbf{b}}=\langle M(\mathbf{b})\rangle+\sum_{\operatorname{dim} \mathcal{O}_{\mathbf{c}}<\operatorname{dim} \mathcal{O}_{\mathbf{b}}} g_{\mathbf{c}}^{\mathbf{b}} E^{\mathbf{c}},
\end{aligned}
$$

where $f_{\mathbf{c}}^{\mathbf{a}}, g_{\mathbf{c}}^{\mathbf{b}} \in \mathcal{A}$ and $\mathbf{c} \in \mathcal{M}$. 
Remark. The conclusion of Lemma 7.1 is also true if we take $M(\mathbf{a})$ to be finitely many copies of an exceptional module.

Lemma 7.2. Let $\pi \in \Pi_{i}^{a}$ for some $\mathcal{T}_{i}$. Then there exists a monomial $\mathfrak{m}_{\pi}$ in the divided powers of $u_{\left[S_{i}\right]}(i \in I)$ such that

$$
\mathfrak{m}_{\pi}=E_{\pi}+\sum_{\operatorname{dim} \mathcal{O}_{\mathbf{c}}<\operatorname{dim} \mathcal{O}_{\pi}} f_{\mathbf{c}}^{\pi} E^{\mathbf{c}}, \quad \text { where } f_{\mathbf{c}}^{\pi} \in \mathcal{A} .
$$

Proof. Let $\left\{\theta_{1}, \ldots, \theta_{r_{i}}\right\}$ be a complete set of non-isomorphic quasi-simple modules of $\mathcal{T}_{i}$ in the natural order (see Section 3). By Proposition 3.2, we then have, in $\mathcal{H}_{q}^{*}(\Lambda)$

$\mathfrak{m}^{\left(w_{\pi}\right)}=E_{\pi}+\sum_{\lambda \in \Pi_{\alpha}^{a}, \lambda \prec \pi} v_{q}^{-\operatorname{dim} M(\pi)+\operatorname{dim} \operatorname{End} M(\pi)+\operatorname{dim} M(\lambda)-\operatorname{dim} \operatorname{End} M(\lambda)} g_{w_{\pi}}^{\lambda}\left(v_{q}^{2}\right) E_{\lambda}$,

where $\mathfrak{m}^{\left(w_{\pi}\right)}=\theta_{j_{1}}^{\left(* e_{1}\right)} * \cdots * \theta_{j_{t}}^{\left(* e_{t}\right)}$. Since each $\theta_{j_{i}}$ is an exceptional module, we have $\left.\left\langle u_{\left[\theta_{j_{p}}\right]}\right\rangle\right\rangle^{\left.* e_{p}\right)}=\left\langle e_{p} \theta_{j_{p}}\right\rangle$ (see the proof in $\S 4.2$ ).

Let $\pi_{j_{p}} \in \Pi_{i}^{a}$ be such that $M\left(\pi_{j_{p}}\right)=e_{p} \theta_{j_{p}}$ and $\underline{\operatorname{dim}} M\left(\pi_{j_{p}}\right)=\left(d_{1}, \ldots, d_{n}\right)$ with $I$ ordered as in $\S 4.4$. By Lemma 7.1 and the subsequent remark, we define a monomial $\mathfrak{m}_{j_{p}}$ such that

$$
\mathfrak{m}_{j_{p}}=\left\langle S_{1}\right\rangle^{\left(* d_{1}\right)} * \cdots *\left\langle S_{n}\right\rangle^{\left(* d_{n}\right)}=\left\langle M\left(\pi_{j_{p}}\right)\right\rangle+\sum_{\operatorname{dim} \mathcal{O}_{\mathbf{c}}<\operatorname{dim} \mathcal{O}_{M\left(\pi_{j_{p}}\right)}} f_{\mathbf{c}}^{\pi_{j_{p}}} E^{\mathbf{c}}
$$

where $f_{\mathbf{c}}^{\pi_{j_{p}}} \in \mathbb{Q}\left[v, v^{-1}\right]$.

Let $L_{1}=e_{1} \theta_{j_{1}}, L_{2}=L_{1} \diamond e_{2} \theta_{j_{2}}, \ldots, L_{t}=L_{t-1} \diamond e_{t} \theta_{j_{t}} \in \mathcal{T}_{i}$. By Lemma 2.3(vi), we have $M(\pi) \simeq L_{t}$. Similar to the argument as in $\S 3.3$, we have $g_{L_{p-1}, e_{j_{p}} \theta_{j_{p}}}^{L_{p}}=1$ for $2 \leq p \leq t$. Define $\alpha_{p}=\underline{\operatorname{dim}} L_{p-1}$ and $\beta_{p}=\underline{\operatorname{dim}} M\left(\pi_{j_{p}}\right)$. By Lemma 2.3(vi),

$$
\operatorname{dim} \mathcal{O}_{L_{p}}=\operatorname{dim} \mathcal{O}_{L_{p-1}}+\operatorname{dim} \mathcal{O}_{e_{p} \theta_{j_{p}}}+\mathbf{m}\left(\alpha_{p}, \beta_{p}\right)
$$

or

$$
\operatorname{codim} \mathcal{O}_{L_{p}}=\operatorname{codim} \mathcal{O}_{L_{p-1}}+\operatorname{codim} \mathcal{O}_{e_{p} \theta_{j_{p}}}-\left\langle\beta_{p}, \alpha_{p}\right\rangle
$$

Thus

$$
\operatorname{dim} \mathcal{O}_{M(\pi)}=\operatorname{dim} \mathcal{O}_{L_{t}}=\sum_{p=1}^{t} \operatorname{dim} \mathcal{O}_{e_{p} \theta_{j_{p}}}+\sum_{p=2}^{t} \mathbf{m}\left(\alpha_{p}, \beta_{p}\right)
$$

For any $\mathbf{c} \in \mathcal{M}$ with $\mathcal{O}_{\mathbf{c}} \subseteq \mathbb{E}_{e_{p} \theta_{j_{p}}}$ and $\operatorname{dim} \mathcal{O}_{\mathbf{c}}<\operatorname{dim} \mathcal{O}_{e_{p} \theta_{j_{p}}}$, by Lemma 2.2,

$$
\begin{aligned}
\operatorname{codim} \overline{\mathcal{O}}_{L_{p-1}} \star \overline{\mathcal{O}_{\mathbf{c}}} & =\operatorname{codim} \overline{\mathcal{O}}_{L_{p-1}}+\operatorname{codim} \overline{\mathcal{O}_{\mathbf{c}}}-\left\langle\beta_{p}, \alpha_{p}\right\rangle+r \\
& >\operatorname{codim} \overline{\mathcal{O}}_{L_{p-1}}+\operatorname{codim} \mathcal{O}_{e_{p} \theta_{j_{p}}}-\left\langle\beta_{p}, \alpha_{p}\right\rangle=\operatorname{codim} \mathcal{O}_{L_{p}} .
\end{aligned}
$$


If we take $\mathfrak{m}_{\pi}=\mathfrak{m}_{\pi_{j_{1}}} * \cdots * \mathfrak{m}_{\pi_{j_{t}}}$, then

$$
\begin{aligned}
\mathfrak{m}_{\pi}= & \left(\left\langle\theta_{j_{1}}\right\rangle^{\left(* e_{1}\right)}+\sum_{\operatorname{dim} \mathcal{O}_{\mathbf{c}_{1}}<\operatorname{dim} \mathcal{O}_{e_{1} \theta_{j_{1}}}} f_{\mathbf{c}_{1}}^{\pi_{j_{1}}} E^{\mathbf{c}_{1}}\right) \\
& * \cdots *\left(\left\langle\theta_{j_{t}}\right\rangle^{\left(* e_{t}\right)}+\sum_{\operatorname{dim} \mathcal{O}_{\mathbf{c}_{t}}<\operatorname{dim} \mathcal{O}_{e_{t} \theta_{j_{t}}}} f_{\mathbf{c}_{t}}^{\pi_{j_{t}}} E^{\mathbf{c}_{t}}\right) \\
= & \left(\left\langle M\left(\pi_{j_{1}}\right)\right\rangle+\sum_{\operatorname{dim} \mathcal{O}_{\mathbf{c}_{1}}<\operatorname{dim} \mathcal{O}_{e_{1} \theta_{j_{1}}}} f_{\mathbf{c}_{1}}^{\pi_{j_{1}}} E^{\mathbf{c}_{1}}\right) \\
& * \cdots *\left(\left\langle M\left(\pi_{j_{t}}\right)\right\rangle+\sum_{\operatorname{dim} \mathcal{O}_{\mathbf{c}_{t}}<\operatorname{dim} \mathcal{O}_{e_{t} \theta_{j_{t}}}} f_{\mathbf{c}_{t}}^{\pi_{j_{t}}} E^{\mathbf{c}_{t}}\right) \\
= & E_{\pi}+\sum_{\operatorname{dim} \mathcal{O}_{\mathbf{c}}<\operatorname{dim} \mathcal{O}_{\pi}} f_{\mathbf{c}}^{\pi} E^{\mathbf{c}}, \quad \text { where } f_{\mathbf{c}}^{\pi} \in \mathcal{A} .
\end{aligned}
$$

Lemma 7.3. Let $E_{n \delta}$ be the embedded image in $\mathcal{C}^{*}(\Lambda)_{\mathcal{A}}$ of the element $E_{n \delta_{K}}$ in $\mathcal{C}^{*}(K)_{\mathcal{A}}$ as in $\S 6.1$. Then there exists a monomial $\mathfrak{m}_{n \delta}$ in the divided powers of $u_{\left[S_{i}\right]}(i \in I)$ such that

$$
\mathfrak{m}_{n \delta}=E_{n \delta}+\sum_{\operatorname{dim} \mathcal{O}_{\mathbf{c}}<\operatorname{dim} \mathcal{O}_{n \delta}} h_{\mathbf{c}}^{n \delta} E^{\mathbf{c}}, \quad \text { where } h_{\mathbf{c}}^{n \delta} \in \mathcal{A} .
$$

Proof. We let $\theta_{1}, \theta_{2}$ be the two simple objects of $\mathfrak{C}(P, L)$. By Lemma 5.11(iii),

$$
\left\langle\theta_{2}\right\rangle^{(* n)} *\left\langle\theta_{1}\right\rangle^{(* n)}=E_{n \delta}+\sum_{\operatorname{dim} \mathcal{O}_{\mathbf{c}}<\operatorname{dim} \mathcal{O}_{n \delta}} f_{\mathbf{c}}^{n \delta} E^{\mathbf{c}} \quad \text { with } f_{\mathbf{c}}^{n \delta} \in \mathbb{Q}\left[v, v^{-1}\right] .
$$

Suppose that $\underline{\operatorname{dim}} n \theta_{1}=\mathbf{d}^{\prime}=\left(d_{1}^{\prime}, \ldots, d_{n}^{\prime}\right)$ and $\underline{\operatorname{dim}} n \theta_{2}=\mathbf{d}^{\prime \prime}=\left(d_{1}^{\prime \prime}, \ldots, d_{n}^{\prime \prime}\right)$ in $\mathbb{Z} I$. Since $\theta_{1}$ and $\theta_{2}$ are exceptional modules, by the remark following Lemma 7.1, we then have

$$
\begin{aligned}
& \mathfrak{m}_{1}=\left\langle S_{1}\right\rangle^{\left(* d_{1}^{\prime}\right)} * \cdots *\left\langle S_{n}\right\rangle^{\left(* d_{n}^{\prime}\right)}=\left\langle\theta_{1}\right\rangle^{(* n)}+\sum_{\operatorname{dim} \mathcal{O}_{\mathbf{c}}<\operatorname{dim} \mathcal{O}_{n \theta_{1}}} f_{\mathbf{c}}^{n \theta_{1}} E^{\mathbf{c}}, \\
& \mathfrak{m}_{2}=\left\langle S_{1}\right\rangle^{\left(* d_{1}^{\prime \prime}\right)} * \cdots *\left\langle S_{n}\right\rangle^{\left(* d_{n}^{\prime \prime}\right)}=\left\langle\theta_{2}\right\rangle^{(* n)}+\sum_{\operatorname{dim} \mathcal{O}_{\mathbf{c}}<\operatorname{dim} \mathcal{O}_{n \theta_{2}}} g_{\mathbf{c}}^{n \theta_{2}} E^{\mathbf{c}},
\end{aligned}
$$

where $f_{\mathbf{c}}^{n \theta_{1}}, g_{\mathbf{c}}^{n \theta_{2}} \in \mathbb{Q}\left[v, v^{-1}\right]$. By representations of the Kronecker quiver, we know that $\mathcal{N}_{n \delta}$ is open in $\mathcal{O}_{n \theta_{2}} \star \mathcal{O}_{n \theta_{1}}$. Moreover, $\mathcal{N}_{n \delta}$ is open and so dense in $\overline{\mathcal{O}}_{n \theta_{2}} \star \overline{\mathcal{O}}_{n \theta_{1}}$, that is, $\mathcal{N}_{n \delta}$ is an irreducible $G$-stable open subvariety of $\overline{\mathcal{O}}_{n \theta_{2}} \star \overline{\mathcal{O}}_{n \theta_{1}}$ (of course with maximal dimension). By Lemma 2.2, we then obtain

$$
\operatorname{codim} \overline{\mathcal{O}}_{n \theta_{2}} \star \overline{\mathcal{O}}_{n \theta_{1}}=\operatorname{codim} \overline{\mathcal{O}}_{n \theta_{2}}+\operatorname{codim} \overline{\mathcal{O}}_{n \theta_{1}}-\left\langle\mathbf{d}^{\prime}, \mathbf{d}^{\prime \prime}\right\rangle
$$


If either $\mathcal{O}_{\mathbf{c}} \subset \overline{\mathcal{O}}_{n \theta_{2}} \backslash \mathcal{O}_{n \theta_{2}}$ or $\mathcal{O}_{\mathbf{c}^{\prime}} \subset \overline{\mathcal{O}}_{n \theta_{1}} \backslash \mathcal{O}_{n \theta_{1}}$, then

$$
\begin{aligned}
\operatorname{codim} \overline{\mathcal{O}}_{\mathbf{c}} \star \overline{\mathcal{O}}_{\mathbf{c}^{\prime}} & =\operatorname{codim} \overline{\mathcal{O}}_{\mathbf{c}}+\operatorname{codim} \overline{\mathcal{O}}_{\mathbf{c}^{\prime}}-\left\langle\mathbf{d}^{\prime}, \mathbf{d}^{\prime \prime}\right\rangle+r \\
& >\operatorname{codim} \overline{\mathcal{O}}_{n \theta_{2}} \star \overline{\mathcal{O}}_{n \theta_{1}}=\operatorname{codim} \mathcal{N}_{n \delta} .
\end{aligned}
$$

We now take $\mathfrak{m}_{n \delta}=\mathfrak{m}_{2} * \mathfrak{m}_{1}$. Then

$$
\begin{aligned}
\mathfrak{m}_{n \delta} & =\left(\left\langle\theta_{2}\right\rangle^{(* n)}+\sum_{\operatorname{dim} \mathcal{O}_{\mathbf{c}}<\operatorname{dim} \mathcal{O}_{n \theta_{2}}} g_{\mathbf{c}}^{n \delta} E^{\mathbf{c}}\right) *\left(\left\langle\theta_{1}\right\rangle^{(* n)}+\sum_{\operatorname{dim} \mathcal{O}_{\mathbf{c}^{\prime}}<\operatorname{dim} \mathcal{O}_{n \theta_{1}}} f_{\mathbf{c}^{\prime}}^{n \delta} E^{\mathbf{c}^{\prime}}\right) \\
& =E_{n \delta}+\sum_{\operatorname{dim} \mathcal{O}_{\mathbf{c}}<\operatorname{dim} \mathcal{O}_{n \delta}} h_{\mathbf{c}}^{n \delta} E^{\mathbf{c}}, \quad \text { where } h_{\mathbf{c}}^{n \delta} \in \mathcal{A} .
\end{aligned}
$$

Proposition 7.4. For any $E^{\mathbf{c}}, \mathbf{c} \in \mathcal{M}$, there exists a monomial $\mathfrak{m}_{\mathbf{c}}$ in the divided powers of $u_{\left[S_{i}\right]}, i \in I$, such that

$$
\mathfrak{m}_{\mathbf{c}}=E^{\mathbf{c}}+\sum_{\mathbf{c}^{\prime} \in \mathcal{M}, \operatorname{dim} \mathcal{O}_{\mathbf{c}^{\prime}}<\operatorname{dim} \mathcal{O}_{\mathbf{c}}} h_{\mathbf{c}^{\prime}}^{\mathbf{c}} E^{\mathbf{c}^{\prime}},
$$

where $h_{\mathbf{c}^{\prime}}^{\mathbf{c}} \in \mathcal{A}$.

Proof. Using the property of the Auslander-Reiten quiver of a tame quiver, if $P \in$ Prep, $I \in$ Prei and $R$ is a regular module, Lemma 2.3(vii) implies that $\mathcal{O}_{P \oplus R \oplus I}$ is open in $\overline{\mathcal{O}}_{P} \star \overline{\mathcal{O}}_{R} \star \overline{\mathcal{O}}_{I}$. Thus we need to prove the same property for $E_{\pi} * E_{n \delta}$ where $\pi \in \Pi_{i}^{a}$. By Lemmas 7.2 and 7.3 , there exist $\mathfrak{m}_{\pi}$ and $\mathfrak{m}_{n \delta}$ such that

$$
\mathfrak{m}_{\pi}=E_{\pi}+\sum_{\operatorname{dim} \mathcal{O}_{\mathbf{c}}<\operatorname{dim} \mathcal{O}_{\pi}} f_{\mathbf{c}}^{\pi} E^{\mathbf{c}}, \quad \mathfrak{m}_{n \delta}=E_{n \delta}+\sum_{\operatorname{dim} \mathcal{O}_{\mathbf{c}^{\prime}}<\operatorname{dim} \mathcal{O}_{n \delta}} g_{\mathbf{c}^{\prime}}^{n \delta} E^{\mathbf{c}^{\prime}},
$$

where $f_{\mathbf{c}}^{n \delta}, g_{\mathbf{c}^{\prime}}^{n \delta} \in \mathcal{A}$.

Since we can find smooth points $A \in \overline{\mathcal{O}}_{\pi}$ and $B \in \overline{\mathcal{O}}_{n \delta}$ such that $\operatorname{Hom}(B, A)$ $=0$, by Lemma 2.2 we have

$$
\operatorname{codim} \overline{\mathcal{O}}_{\pi} \star \overline{\mathcal{O}}_{n \delta}=\operatorname{codim} \overline{\mathcal{O}}_{\pi}+\operatorname{codim} \overline{\mathcal{O}}_{n \delta}-\langle n \delta, \alpha\rangle .
$$

If either $\mathcal{O}_{\mathbf{c}^{\prime}} \subset \overline{\mathcal{O}}_{\pi} \backslash \mathcal{O}_{\pi}$ or $\mathcal{O}_{\mathbf{c}^{\prime \prime}} \subset \overline{\mathcal{O}}_{n \delta} \backslash \mathcal{O}_{n \delta}$, we have again

$$
\operatorname{codim} \overline{\mathcal{O}}_{\mathbf{c}^{\prime}} \star \overline{\mathcal{O}}_{\mathbf{c}^{\prime \prime}}>\operatorname{codim} \overline{\mathcal{O}}_{\pi} \star \overline{\mathcal{O}}_{n \delta}=\operatorname{codim} \mathcal{O}_{\pi} \star \mathcal{O}_{n \delta},
$$

and

$$
\begin{aligned}
\mathfrak{m}_{\mathbf{c}} & =\mathfrak{m}_{\pi} * \mathfrak{m}_{n \delta} \\
& =\left(E_{\pi}+\sum_{\operatorname{dim} \mathcal{O}_{\mathbf{c}^{\prime}}<\operatorname{dim} \mathcal{O}_{\pi}} f_{\mathbf{c}^{\prime}}^{\pi} E^{\mathbf{c}^{\prime}}\right) *\left(E_{n \delta}+\sum_{\operatorname{dim} \mathcal{O}_{\mathbf{c}^{\prime \prime}}<\operatorname{dim} \mathcal{O}_{n \delta}} g_{\mathbf{c}^{\prime \prime}}^{n \delta} E^{\mathbf{c}^{\prime \prime}}\right) \\
& =E^{\mathbf{c}}+\sum_{\mathbf{c}^{\prime \prime \prime} \in \mathcal{M}, \operatorname{dim} \mathcal{O}_{\mathbf{c}^{\prime \prime \prime}}<\operatorname{dim} \mathcal{O}_{\mathbf{c}}} h_{\mathbf{c}^{\prime \prime \prime}}^{\mathbf{c}} E^{\mathbf{c}^{\prime \prime \prime}}, \quad \text { where } h_{\mathbf{c}^{\prime}}^{\mathbf{c}} \in \mathcal{A} .
\end{aligned}
$$




\section{$\S 7.2$}

We define the lattice $\mathcal{L}^{\prime}$ to be the $\mathbb{Q}\left[v^{-1}\right]$-submodule of $\mathcal{C}^{*}(\Lambda)_{\mathcal{A}}$ with the basis $\left\{E^{\mathbf{c}} \mid \mathbf{c} \in \mathcal{M}\right\}$. By an argument similar to that in [L1], we define $\omega_{\mathbf{c}^{\prime}}^{\mathbf{c}} \in \mathcal{A}$ such that

$$
\overline{E^{\mathbf{c}}}=\sum_{\mathbf{c}^{\prime} \in \mathcal{M}} \omega_{\mathbf{c}^{\prime}}^{\mathbf{c}} E^{\mathbf{c}^{\prime}} \quad \text { for any } \mathbf{c} \in \mathcal{M} .
$$

Then $\omega_{\mathbf{c}^{\prime}}^{\mathbf{c}} \in \mathcal{A}, \omega_{\mathbf{c}}^{\mathbf{c}}=1$ and if $\omega_{\mathbf{c}^{\prime}}^{\mathbf{c}} \neq 0$ and $\mathbf{c} \neq \mathbf{c}^{\prime}$ then $\operatorname{dim} \mathcal{O}_{\mathbf{c}^{\prime}}<\operatorname{dim} \mathcal{O}_{\mathbf{c}}$. Thus we can solve for $\zeta_{\mathbf{c}^{\prime}}^{\mathbf{c}} \in \mathcal{A}$ the system of equations

$$
\zeta_{\mathbf{c}^{\prime}}^{\mathbf{c}}=\sum_{\operatorname{dim} \mathcal{O}_{\mathbf{c}^{\prime}} \leq \operatorname{dim} \mathcal{O}_{\mathbf{c}^{\prime \prime}} \leq \operatorname{dim} \mathcal{O}_{\mathbf{c}}} \omega_{\mathbf{c}^{\prime}}^{\mathbf{c}^{\prime \prime}} \overline{{\overline{\mathbf{c}^{\prime \prime}}}^{\mathbf{c}}}
$$

to get a unique solution such that

$$
\zeta_{\mathbf{c}}^{\mathbf{c}}=1 \quad \text { and } \quad \zeta_{\mathbf{c}^{\prime}}^{\mathbf{c}} \in v^{-1} \mathbb{Q}\left[v^{-1}\right] \quad \text { if } \operatorname{dim} \mathcal{O}_{\mathbf{c}^{\prime}}<\operatorname{dim} \mathcal{O}_{\mathbf{c}} .
$$

For each $\mathbf{c} \in \mathcal{M}$, define

$$
\mathcal{E}^{\mathbf{c}}=\sum_{\mathbf{c}^{\prime} \in \mathcal{M}} \zeta_{\mathbf{c}^{\prime}}^{\mathbf{c}} E^{\mathbf{c}^{\prime}},
$$

which is a finite sum. Then we have the following result.

Theorem 7.5 The set $\mathbf{J}=\left\{\mathcal{E}^{\mathbf{c}} \mid \mathbf{c} \in \mathcal{M}\right\}$ is an $\mathcal{A}$-basis of $\mathcal{C}^{*}(\Lambda)_{\mathcal{A}}$ with the two properties:

(i) $\overline{\mathcal{E}^{\mathbf{c}}}=\mathcal{E}^{\mathbf{c}}$ for all $\mathbf{c} \in \mathcal{M}$,

(ii) $\pi\left(\mathcal{E}^{\mathbf{c}}\right)=\pi\left(E^{\mathbf{c}}\right)$,

where $\pi: \mathcal{L}^{\prime} \rightarrow \mathcal{L}^{\prime} / v^{-1} \mathcal{L}^{\prime}$ is the canonical projection.

Proof. (i) We have

$$
\overline{\mathcal{E}^{\mathbf{c}}}=\sum_{\mathbf{c}^{\prime}} \overline{\zeta_{\mathbf{c}^{\prime}}^{\mathbf{c}}} \overline{E^{\mathbf{c}^{\prime}}}=\sum_{\mathbf{c}^{\prime}} \overline{\zeta_{\mathbf{c}^{\prime}}^{\mathbf{c}}} \sum_{\mathbf{c}^{\prime \prime}} \omega_{c^{\prime \prime}}^{\mathbf{c}^{\prime}} E^{\mathbf{c}^{\prime \prime}}=\sum_{\mathbf{c}^{\prime \prime}}\left(\sum_{\mathbf{c}^{\prime}} \overline{\zeta_{\mathbf{c}^{\prime}}} \omega_{c^{\prime \prime}}^{\mathbf{c}^{\prime}}\right) E^{\mathbf{c}^{\prime \prime}}=\sum_{\mathbf{c}^{\prime \prime}} \zeta_{\mathbf{c}^{\prime \prime}}^{\mathbf{c}} E^{\mathbf{c}^{\prime \prime}}=\mathcal{E}^{\mathbf{c}} .
$$

So the elements $\mathcal{E}^{\mathbf{c}}$ are bar-invariant.

(ii) Obviously the set $\mathbf{J}$ is a $\mathbb{Q}\left[v^{-1}\right]$-basis of the lattice $\mathcal{L}^{\prime}$.

\section{$\S 8 . \quad$ Affine canonical bases}

Recall that $\Lambda=k Q$ and $k=\mathbb{F}_{q}$ is a finite field. For any $\Lambda$-module $M$ and any field extension $k \subseteq K$, we view $M^{K}=M \otimes_{k} K$ as a $K Q$-module. For any regular $\Lambda$-modules $M, \ldots, M_{t}$ and $L$, it is known that there exists the Hall polynomial $\varphi_{M_{1} \cdots M_{t}}^{L} \in \mathbb{Q}[T]$ such that $\varphi_{M_{1} \cdots M_{t}}^{L}\left(q^{n}\right)=g_{M_{1}^{K}, \ldots, M_{t}^{K}}^{L^{K}}$ for any finite extension 
$k \subseteq K$ of degree $n$ ([R2], [Z2]). Similarly, we have a polynomial $a_{M}$ such that $a_{M}\left(q^{n}\right)=\left|\operatorname{Aut}_{K Q}\left(M^{K}\right)\right|$. Therefore, we will perform computations in the Hall algebra $\mathcal{H}_{q}(\Lambda)$ and then use the argument as in $\S 1.4$ to get a generic form.

\section{$\S 8.1$}

The Ringel-Hall algebra $\mathcal{H}_{q}^{*}(\Lambda)$ is an associative $\mathbb{Q}\left(v_{q}\right)$-algebra with the basis $\{\langle M\rangle \mid M \in \mathcal{P}\}$. Note that $\langle M\rangle=v_{q}^{-\operatorname{dim} M+\operatorname{dim} \operatorname{End}_{\Lambda}(M)} u_{[M]}$. An inner product $(-,-)_{q}$ on $\mathcal{H}_{q}^{*}(\Lambda)$ is defined in $[\mathrm{G}]$ by

$$
(\langle M\rangle,\langle N\rangle)_{q}=\delta_{M, N} \frac{v_{q}^{2} \operatorname{dim} \operatorname{End} M}{a_{M}},
$$

where $a_{M}=|\operatorname{Aut}(M)|$. Following Green [G] and Ringel [R1], we can define a linear $\operatorname{map} r_{q}: \mathcal{H}_{q}^{*}(\Lambda) \rightarrow \mathcal{H}_{q}^{*}(\Lambda) \otimes_{\mathbb{Q}\left(v_{q}\right)} \mathcal{H}_{q}^{*}(\Lambda)$ by

$$
r_{q}\left(u_{[L]}\right)=\sum_{[M],[N]} v_{q}^{\langle\underline{\operatorname{dim}} M, \underline{\operatorname{dim}} N\rangle} g_{M, N}^{L} \frac{a_{M} a_{M}}{a_{L}} u_{M} \otimes u_{N} .
$$

We have the following property:

$$
(x, y * z)_{q}=\left(r_{q}(x), y \otimes z\right)_{q} \quad \text { for any } x, y, z \in \mathcal{H}_{q}^{*}(\Lambda) .
$$

Using the fact that $\operatorname{Ext}(P, R)=\operatorname{Ext}(R, I)=\operatorname{Ext}(P, I)=0$ for $P, R$, and $I$ being preprojective, regular, and preinjective respectively, a direct computation shows

Proposition 8.1. For any preprojective $\Lambda$-modules $P, P^{\prime} \in \mathcal{P}$, regular $\Lambda$-modules $R, R^{\prime} \in \mathcal{P}$ and preinjective $\Lambda$-modules $I, I^{\prime} \in \mathcal{P}$, we have, in $\mathcal{H}_{q}^{*}(\Lambda)$,

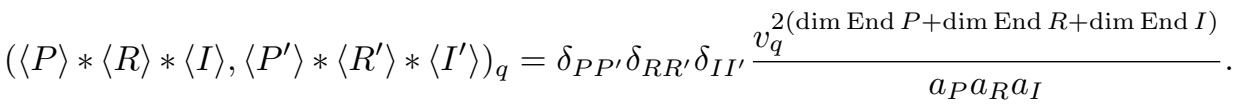

Using the argument of $\S 1.4$, the linear maps $r_{q}$ induce a $\mathcal{Z}$-linear map $r$ : $\mathcal{C}^{*}(\Lambda)_{\mathcal{Z}} \rightarrow \mathcal{C}^{*}(\Lambda)_{\mathcal{Z}} \otimes_{\mathcal{Z}} \mathcal{C}^{*}(\Lambda)_{\mathcal{Z}}$, and the bilinear maps $(-,-)_{q}$ define a $\mathcal{Z}$-bilinear form $(-,-): \mathcal{C}^{*}(\Lambda) \mathcal{Z} \otimes_{\mathcal{Z}} \mathcal{C}^{*}(\Lambda)_{\mathcal{Z}} \rightarrow \mathcal{Z}$. The form $(-,-)$ coincides with the paring defined by Lusztig [L5] under the isomorphism $\mathcal{C}^{*}(\Lambda)_{\mathcal{Z}} \cong U_{\mathcal{Z}}^{+}$. This can be easily verified by comparing the values on simple objects (Chevalley generators) and by using (8.3).

\section{$\S 8.2$}

Now we calculate the inner product on elements in the PBW basis $\left\{E^{c} \mid c \in \mathcal{M}\right\}$. For the Kronecker quiver, it follows from Section 5 (after taking the generic form) 
that, in $\mathcal{C}^{*}(\Lambda)_{\mathcal{Z}}$,

$$
\begin{aligned}
& \tilde{E}_{n \delta}=E_{(n-1, n)} * E_{1}-v^{-2} E_{1} * E_{(n-1, n)}, \\
& E_{0 \delta}=1, \quad E_{n \delta}=\frac{1}{[n]} \sum_{s=1}^{n} v^{s-n} \tilde{E}_{s \delta} * E_{(n-s) \delta} .
\end{aligned}
$$

Using the calculations in $[\mathrm{BCP}], E_{n \delta}$ corresponds to the complete symmetric function $h_{(n)}$ in $[\mathrm{M}]$ and $E_{\omega_{\mathbf{c}} \delta}$ corresponds to the complete symmetric functions $h_{\omega_{\mathbf{c}}}$ in [M] (see Sections 1 and 3 in [BCP]). Then

$$
\begin{gathered}
\left(E_{n \delta}, E_{n \delta}\right) \equiv 1\left(\bmod v^{-1} \mathbb{Q}\left[\left[v^{-1}\right]\right] \cap \mathbb{Q}(v)\right), \\
\left(E_{n \delta}, E_{\omega_{\mathbf{c}} \delta}\right) \in \mathbb{N}^{*}+v^{-1} \mathbb{Q}\left[\left[v^{-1}\right]\right] \cap \mathbb{Q}(v), \\
\left(E_{\omega_{\mathbf{c}} \delta}, E_{\omega_{\mathbf{c}} \delta}\right) \in \mathbb{N}^{*}+v^{-1} \mathbb{Q}\left[\left[v^{-1}\right]\right] \cap \mathbb{Q}(v),
\end{gathered}
$$

for any $n \geq 0$ and any partition $\omega_{\mathbf{c}}$ of $n$.

Let $F: \mathcal{H}_{q}^{*}(K) \rightarrow \mathcal{H}_{q}^{*}(\Lambda)$ be the embedding and $\mathfrak{C}(P, L)$ be the full subcategory of $\bmod \Lambda$ with two relative simple objects $\theta_{1}, \theta_{2}$ as in $\S 6.1$. We denote by $\mathfrak{C}_{0}$ (resp. $\left.\mathfrak{C}_{1}\right)$ the full subcategory of $\mathfrak{C}(P, L)$ consisting of the $\Lambda$-modules which belong to homogeneous (resp. non-homogeneous) tubes of $\bmod \Lambda$. We will use iso $\mathfrak{C}_{0}$ and iso $\mathfrak{C}_{1}$ to denote the isomorphism classes of objects in $\mathfrak{C}_{0}$ and $\mathfrak{C}_{1}$ respectively. Note that the classes of indecomposable objects in $\mathfrak{C}_{1}$ do not depend on $q$, while the classes of indecomposable objects in $\mathfrak{C}_{0}$ do depend on $q$. By the definition of the inner product in $\S 8.1$, this embedding preserves the inner products. Recall the definition of $E_{n \delta} \in \mathcal{H}_{q}^{*}(\Lambda)$ which is the image of $E_{n \delta} \in \mathcal{H}_{q}^{*}(K)$. Now in $\mathcal{H}_{q}^{*}(\Lambda)$ we have the decomposition

$$
E_{n \delta}=E_{n \delta, 1}+E_{n \delta, 2}+E_{n \delta, 3}
$$

with

$$
\begin{aligned}
& E_{n \delta, 1}=v_{q}^{-n \operatorname{dim} \theta_{1}-n \operatorname{dim} \theta_{2}} \sum_{[M] \in \operatorname{iso}} \sum_{\mathfrak{C}_{1}, \underline{\operatorname{dim}} M=n \delta} u_{[M]}, \\
& E_{n \delta, 2}=v_{q}^{-n \operatorname{dim} \theta_{1}-n \operatorname{dim} \theta_{2}} \sum_{\substack{\operatorname{dim}\left(M_{0}+M_{1}\right)=n \delta \\
[0] \neq\left[M_{1}\right] \in \operatorname{iso} \mathfrak{C}_{1},[0] \neq\left[M_{0}\right] \in \text { iso } \mathfrak{C}_{0}}} u_{\left[M_{0} \oplus M_{1}\right]}, \\
& E_{n \delta, 3}=v_{q}^{-n \operatorname{dim} \theta_{1}-n \operatorname{dim} \theta_{2}} u_{[M] \in \text { iso } \mathfrak{C}_{0}, \underline{\operatorname{dim}} M=n \delta} u_{[M]} .
\end{aligned}
$$

Note that $\operatorname{dim} \theta_{i}(i=1,2)$ is independent of $q$. It is easy to see that $\left(E_{n \delta, i}, E_{n \delta, j}\right)_{q}$ $=0$ for all $i \neq j$. Although in the decomposition above, $E_{n \delta, i}$ varies for $i=2,3$ as $q$ varies (the number of terms will change) we still use $E_{n \delta, i}$ to denote $\left(E_{n \delta, i}\right)$ in $\prod_{q} \mathcal{H}_{q}^{*}(\Lambda)$. 
In the rest of this section we will use the following facts frequently.

Lemma 8.2.1. Let $M$ be a regular module with $\underline{\operatorname{dim}} M=n \delta$, and $M=\bigoplus_{i=1}^{s} M_{i}$ with $M_{i} \in \mathcal{T}_{i}$ for $i=1, \ldots, s$. Then $\underline{\operatorname{dim}} M_{i}=n_{i} \delta$ and $\sum_{i=1}^{s} n_{i}=n$.

Proof. Assume $M=M_{1} \oplus M_{1}^{\prime}$ is a direct sum decomposition with $M_{1} \in \mathcal{T}_{1}$ and $M_{1}^{\prime}$ having no direct summand in $\mathcal{T}_{1}$. If $\underline{\operatorname{dim}} M_{1}=m_{1} \delta+\beta_{1}$ with $\beta_{1} \notin \mathbb{N} \delta$ and $\underline{\operatorname{dim}} M_{1}^{\prime}=m_{1}^{\prime} \delta+\beta_{1}^{\prime}$ with $\beta_{1}^{\prime} \notin \mathbb{N} \delta$, then $\beta_{1}+\beta_{1}^{\prime} \in \mathbb{N} \delta$. Since $0=\left(m_{1} \delta+\beta_{1}, m_{1}^{\prime} \delta+\right.$ $\left.\beta_{1}^{\prime}\right)=\left(\beta_{1}, \beta_{1}^{\prime}\right)=\left(m \delta-\beta_{1}^{\prime}, \beta_{1}^{\prime}\right)=-\left(\beta_{1}^{\prime}, \beta_{1}^{\prime}\right)$, we get $\beta_{1}^{\prime}=k \delta, k \in \mathbb{N}$. This is a contradiction. Now the lemma follows by induction on $s$.

Lemma 8.2.2. The following relations hold in $\mathcal{C}^{*}(\Lambda)_{\mathcal{A}}$ :

$$
\begin{aligned}
& \left(E_{n \delta, 1}, E_{n \delta, 1}\right) \equiv 0\left(\bmod v^{-1} \mathbb{Q}\left[\left[v^{-1}\right]\right] \cap \mathbb{Q}(v)\right), \\
& \left(E_{n \delta, 2}, E_{n \delta, 2}\right) \equiv 0\left(\bmod v^{-1} \mathbb{Q}\left[\left[v^{-1}\right]\right] \cap \mathbb{Q}(v)\right), \\
& \left(E_{n \delta, 3}, E_{n \delta, 3}\right) \equiv 1\left(\bmod v^{-1} \mathbb{Q}\left[\left[v^{-1}\right]\right] \cap \mathbb{Q}(v)\right) .
\end{aligned}
$$

Proof. We first work over finite fields $\mathbb{F}_{q}$ and then pass to the generic form. Note that $u_{[M]}=v_{q}^{\operatorname{dim} M-\operatorname{dim} \text { End } M}\langle M\rangle$ and $\operatorname{dim} M=n\left(\operatorname{dim} \theta_{1}+\operatorname{dim} \theta_{2}\right)$. Then

$$
E_{n \delta, 1}=\sum_{[M] \in \text { iso }} \sum_{\mathfrak{C}_{1}, \underline{\operatorname{dim}} M=n \delta} v_{q}^{-\operatorname{dim} \operatorname{End} M}\langle M\rangle .
$$

By Proposition 8.1 we have

$$
\left(E_{n \delta, 1}, E_{n \delta, 1}\right)_{q}=\sum_{M \in \operatorname{iso}\left(\mathfrak{C}_{1}\right), \underline{\operatorname{dim}} M=n \delta} v_{q}^{-2 \operatorname{dim} \operatorname{End} M}(\langle M\rangle,\langle M\rangle)_{q} .
$$

Note that $(\langle M\rangle,\langle M\rangle)_{q}=\mid$ End $M \mid / a_{M} \in \mathbb{Q}\left[\left[v_{q}^{-1}\right]\right] \cap \mathbb{Q}\left(v_{q}\right)$, $\mid$ End $M \mid=v_{q}^{2} \operatorname{dim} \operatorname{End} M$ and $a_{M}$ is a polynomial in $v_{q}$ with leading term $v_{q}^{2} \operatorname{dim} \operatorname{End} M$ (here $\operatorname{dim} \operatorname{End} M$ is invariant as $q$ varies). Then

$$
(\langle M\rangle,\langle M\rangle)_{q} \in 1+v_{q}^{-1} \mathbb{Q}\left[\left[v_{q}^{-1}\right]\right] \cap \mathbb{Q}\left(v_{q}\right)
$$

and

$$
\left(E_{n \delta, 1}, E_{n \delta, 1}\right)_{q} \in v_{q}^{-1} \mathbb{Q}\left[\left[v_{q}^{-1}\right]\right] \cap \mathbb{Q}\left(v_{q}\right) .
$$

We now use induction on $n$. Obviously, the relations of the lemma hold for $n=1$. We assume now that they are also true for all $m$ with $m<n$. Since

$$
\begin{aligned}
& E_{n \delta, 2}=v_{q}^{-n \operatorname{dim} \theta_{1}-n \operatorname{dim} \theta_{2}} \sum_{\substack{0 \neq\left[M_{1}\right] \in \operatorname{iso} \\
\underline{\operatorname{dim}}\left(M_{0} \oplus M_{1}\right)=n \delta}} u_{\left[M_{0} \oplus M_{1}\right]} \\
& =\sum_{\substack{\left[M_{1}\right] \in \text { iso } \mathfrak{C}_{1} \\
\operatorname{dim} M_{1}=m \delta, 0<m<n}} v_{q}^{-\operatorname{dim} \text { End } M_{1}}\left\langle M_{1}\right\rangle * E_{(n-m) \delta, 3}
\end{aligned}
$$


(noting that $0<n-m<n$ ), we have

$$
=\sum_{\substack{\left[M_{1}\right] \in \operatorname{iso} \mathfrak{C}_{1} \\ \underline{\operatorname{dim}} M_{1}=m \delta, 0<m<n}}^{\left(E_{n \delta, 2}, E_{n \delta, 2}\right)_{q}} v_{q}^{-2 \operatorname{dim} \operatorname{End} M_{1}}\left(\left\langle M_{1}\right\rangle,\left\langle M_{1}\right\rangle\right)_{q}\left(E_{\left(n \delta-\underline{\operatorname{dim}} M_{1}\right), 3}, E_{\left(n \delta-\underline{\operatorname{dim}} M_{1}\right), 3}\right)_{q} .
$$

Since $\operatorname{dim}$ End $M_{1} \geq 1$ and, by the inductive assumption, $\left(E_{m \delta, 3}, E_{m \delta, 3}\right)_{q} \equiv 1$, we have

$$
\left(E_{n \delta, 2}, E_{n \delta, 2}\right)_{q} \equiv 0\left(\bmod v_{q}^{-1} \mathbb{Q}\left[\left[v_{q}^{-1}\right]\right] \cap \mathbb{Q}\left(v_{q}\right)\right)
$$

for all $n>0$. Since

$$
\left(E_{n \delta}, E_{n \delta}\right)_{q} \equiv 1\left(\bmod v_{q}^{-1} \mathbb{Q}\left[\left[v_{q}^{-1}\right]\right] \cap \mathbb{Q}\left(v_{q}\right)\right)
$$

and

$$
\left(E_{n \delta}, E_{n \delta}\right)_{q}=\left(E_{n \delta, 1}, E_{n \delta, 1}\right)_{q}+\left(E_{n \delta, 2}, E_{n \delta, 2}\right)_{q}+\left(E_{n \delta, 3}, E_{n \delta, 3}\right)_{q},
$$

using the results proved for $E_{n \delta, 1}$ and $E_{n \delta, 2}$ we have

$$
\left(E_{n \delta, 3}, E_{n \delta, 3}\right)_{q} \equiv 1\left(\bmod v_{q}^{-1} \mathbb{Q}\left[\left[v_{q}^{-1}\right]\right] \cap \mathbb{Q}\left(v_{q}\right)\right) .
$$

Now the desired relations hold for all $n$ and $q$. By taking the inner product in $\prod_{q} \mathcal{H}_{q}^{*}(\Lambda)$, the generic form of the lemma follows.

\section{$\S 8.3$}

In the following, we will define a decomposition of the regular part of $\mathcal{C}^{*}(\Lambda)_{\mathcal{A}}$ with respect to the inner product $(-,-)$.

In Section 6, we have constructed the $\mathbb{Q}(v)$-basis $\left\{E^{\mathbf{c}} \mid \mathbf{c} \in \mathcal{M}\right\}$ of $\mathcal{C}^{*}(\Lambda)_{\mathbb{Q}(v)}$. Let $\mathcal{R}\left(\mathcal{C}^{*}(\Lambda)\right)$ be the $\mathbb{Q}(v)$-subspace of $\mathcal{C}^{*}(\Lambda)_{\mathbb{Q}(v)}$ with the basis

$\left\{E_{\pi_{1 \mathbf{c}}} * \cdots * E_{\pi_{s \mathbf{c}}} * E_{w_{\mathbf{c}} \delta} \mid \pi_{\mathbf{c}}=\left(\pi_{1 \mathbf{c}}, \ldots, \pi_{s \mathbf{c}}\right) \in \Pi_{1}^{a} \times \cdots \times \Pi_{s}^{a}\right.$ and $w_{\mathbf{c}}$ is a partition $\}$.

Obviously, $\mathcal{R}\left(\mathcal{C}^{*}(\Lambda)\right)$ is a subalgebra of $\mathcal{C}^{*}(\Lambda)$. Naturally, we take $E_{w_{\mathbf{c}} \delta}=1$ if $w_{\mathbf{c}}=0$.

Let $\mathcal{R}^{a}\left(\mathcal{C}^{*}(\Lambda)\right)$ be the $\mathbb{Q}(v)$-subalgebra of $\mathcal{R}\left(\mathcal{C}^{*}(\Lambda)\right)$ generated by the basis

$$
\left\{E_{\pi_{1 \mathbf{c}}} * \cdots * E_{\pi_{s \mathbf{c}}} \mid \pi_{\mathbf{c}}=\left(\pi_{1 \mathbf{c}}, \ldots, \pi_{s \mathbf{c}}\right) \in \Pi_{1}^{a} \times \cdots \times \Pi_{s}^{a}\right\} .
$$

For $\alpha, \beta \in \mathbb{N}[I]$, we write $\alpha \leq \beta$ if $\beta-\alpha \in \mathbb{N}[I]$. Recall that $\mathcal{C}^{*}(\Lambda)_{\beta}$ is the $\beta$-homogeneous part of the $\mathbb{N} I$-graded algebra. It follows that $\mathcal{R}^{a}\left(\mathcal{C}^{*}(\Lambda)\right)_{\beta}=$ $\mathcal{R}\left(\mathcal{C}^{*}(\Lambda)\right)_{\beta}$ for $\beta<\delta$. We now define

$$
\mathcal{F}_{\delta}=\left\{x \in \mathcal{R}\left(\mathcal{C}^{*}(\Lambda)\right)_{\delta} \mid\left(x, \mathcal{R}^{a}\left(\mathcal{C}^{*}(\Lambda)\right)_{\delta}\right)=0\right\} .
$$


By Proposition 8.1, $(-,-)$ is non-degenerate on $\mathcal{R}\left(\mathcal{C}^{*}(\Lambda)\right)$. Using Lemma 8.2.1, we get

$$
\mathcal{R}\left(\mathcal{C}^{*}(\Lambda)\right)_{\delta}=\mathcal{R}^{a}\left(\mathcal{C}^{*}(\Lambda)\right)_{\delta} \oplus \mathcal{F}_{\delta} \quad \text { and } \quad \operatorname{dim} \mathcal{F}_{\delta}=1
$$

Using Schmidt orthogonalization, we set

$$
E_{\delta}^{\prime}=E_{\delta}-\sum_{M\left(\pi_{i \mathbf{c}}\right), \underline{\operatorname{dim}} M\left(\pi_{i \mathbf{c}}\right)=\delta, 1 \leq i \leq s} a_{\pi_{i \mathbf{c}}} E_{\pi_{i \mathbf{c}}}
$$

satisfying the condition $\mathcal{F}_{\delta}=\mathbb{Q}(v) E_{\delta}^{\prime}$. Now let $\mathcal{R}\left(\mathcal{C}^{*}(\Lambda)\right)(1)$ be the subalgebra of $\mathcal{R}\left(\mathcal{C}^{*}(\Lambda)\right)$ generated by $\mathcal{R}^{a}\left(\mathcal{C}^{*}(\Lambda)\right)$ and $\mathcal{F}_{\delta}$. We have $\mathcal{R}\left(\mathcal{C}^{*}(\Lambda)\right)(1)_{\beta}=\mathcal{R}\left(\mathcal{C}^{*}(\Lambda)\right)_{\beta}$ if $\beta<2 \delta$. Define

$$
\mathcal{F}_{2 \delta}=\left\{x \in \mathcal{R}\left(\mathcal{C}^{*}(\Lambda)\right)_{2 \delta} \mid\left(x, \mathcal{R}\left(\mathcal{C}^{*}(\Lambda)\right)(1)_{2 \delta}\right)=0\right\}
$$

Then $\operatorname{dim} \mathcal{F}_{2 \delta}=1$ and $\mathcal{R}\left(\mathcal{C}^{*}(\Lambda)\right)_{2 \delta}=\mathcal{R}\left(\mathcal{C}^{*}(\Lambda)\right)(1)_{2 \delta} \oplus \mathcal{F}_{2 \delta}$. Recursively, we define

$$
\mathcal{F}_{n \delta}=\left\{x \in \mathcal{R}\left(\mathcal{C}^{*}(\Lambda)\right)_{n \delta} \mid\left(x, \mathcal{R}\left(\mathcal{C}^{*}(\Lambda)\right)(n-1)_{n \delta}\right)=0\right\}
$$

and $\mathcal{R}\left(\mathcal{C}^{*}(\Lambda)\right)(n)$ to be the subalgebra of $\mathcal{R}\left(\mathcal{C}^{*}(\Lambda)\right)$ generated by $\mathcal{R}\left(\mathcal{C}^{*}(\Lambda)\right)(n-1)$ and $\mathcal{F}_{n \delta}$. We have $\mathcal{R}\left(\mathcal{C}^{*}(\Lambda)\right)_{n \delta}=\mathcal{R}\left(\mathcal{C}^{*}(\Lambda)\right)(n-1)_{n \delta} \oplus \mathcal{F}_{n \delta}$ with $\operatorname{dim} \mathcal{F}_{n \delta}=1$. Also, we can choose $E_{n \delta}^{\prime}$ such that $E_{n \delta}-E_{n \delta}^{\prime} \in \mathcal{R}\left(\mathcal{C}^{*}(\Lambda)\right)(n-1)_{n \delta}$ and $\mathcal{F}_{n \delta}=\mathbb{Q}(v) E_{n \delta}^{\prime}$ for all $n>0$.

Lemma 8.3.1. Let $M, N, L$ be regular $\Lambda$-modules with $\underline{\operatorname{dim}} M, \underline{\operatorname{dim}} N, \underline{\operatorname{dim}} L \in$ $\mathbb{N} \delta$. Then the degree of the Hall polynomial $\varphi_{M N}^{L}$ is no more than $\operatorname{dim} \operatorname{End} L-$ (dim End $M+\operatorname{dim}$ End $N$ ).

Proof. By the remark at the beginning of Section 8, we have the Hall polynomial $\varphi_{M N}^{L}$ for $g_{M N}^{L}$, and the polynomials for $a_{M}, a_{N}, a_{L}$, and $|\operatorname{Hom}(M, N)|$. Therefore, we have a rational function, denoted by $f$, such that $f\left(q^{e}\right)=$ $\left|\operatorname{Ext}^{1}\left(M \otimes_{\mathbb{F}_{q}} \mathbb{F}_{q^{e}}, N \otimes_{\mathbb{F}_{q}} \mathbb{F}_{q^{e}}\right)_{L \otimes_{\mathbb{F}_{q}} \mathbb{F}_{q}}\right|$ for all $e \geq 1$. Since $f\left(q^{e}\right)$ is an integer for all $e \geq 1, f$ is a polynomial with coefficients in $\mathbb{Q}$. Since $\langle\underline{\operatorname{dim}} M, \underline{\operatorname{dim}} N\rangle=0$, we have $\operatorname{dim} \operatorname{Ext}^{1}(M, N)=\operatorname{dim} \operatorname{Hom}(M, N)$. The degree of the polynomial $f$ is no more than $\operatorname{dim} \operatorname{Ext}^{1}(M, N)$. Thus,

$$
\operatorname{deg} \varphi_{M N}^{L} \leq \operatorname{deg} a_{L}-\left(\operatorname{deg} a_{M}+\operatorname{deg} a_{N}\right) .
$$

It is also known that $\operatorname{deg} a_{X}=\operatorname{dim}_{k}$ End $X$ for any $\Lambda$-module $X$. This completes the proof. 
Let $w_{\mathbf{c}}=\left(w_{1}, \ldots, w_{t}\right)$ be a partition of $n$. Then

$E_{w_{\mathbf{c}} \delta}=E_{w_{1} \delta} * \cdots * E_{w_{t} \delta}=\left(E_{w_{1} \delta, 1}+E_{w_{1} \delta, 2}+E_{w_{1} \delta, 3}\right) * \cdots *\left(E_{w_{t} \delta, 1}+E_{w_{t} \delta, 2}+E_{w_{t} \delta, 3}\right)$.

We set $E_{w_{\mathbf{c}} \delta, 3}=E_{w_{1} \delta, 3} * \cdots * E_{w_{t} \delta, 3}$.

Lemma 8.3.2. Let $w_{\mathbf{c}}$ be a partition of $n$. Then

$$
\left(E_{n \delta}, E_{w_{\mathbf{c}} \delta}\right) \equiv\left(E_{n \delta, 3}, E_{w_{\mathbf{c}} \delta, 3}\right)\left(\bmod v^{-1} \mathbb{Q}\left[\left[v^{-1}\right]\right] \cap \mathbb{Q}(v)\right) .
$$

Proof. We use induction on $n$. When $n=1$, the result follows from Lemma 8.2.2. Suppose

$$
\left(E_{m \delta}, E_{w_{\mathbf{c}}^{\prime} \delta}\right) \equiv\left(E_{m \delta, 3}, E_{w_{\mathbf{c}}^{\prime} \delta, 3}\right)\left(\bmod v^{-1} \mathbb{Q}\left[\left[v^{-1}\right]\right] \cap \mathbb{Q}(v)\right)
$$

for all partitions $w_{\mathbf{c}}^{\prime}$ of $m$ with $m<n$. Let $w_{\mathbf{c}}$ be a partition of $n$. Since $E_{k \delta}=$ $E_{k \delta, 1}+E_{k \delta, 2}+E_{k \delta, 3}$ for any $k \in \mathbb{N}$, we have, in $\mathcal{H}_{q}^{*}(\Lambda)$,

$$
\begin{aligned}
E_{w_{\mathbf{c}} \delta} & =\left(E_{w_{1} \delta, 1}+E_{w_{1} \delta, 2}+E_{w_{1} \delta, 3}\right) * \cdots *\left(E_{w_{t} \delta, 1}+E_{w_{t} \delta, 2}+E_{w_{t} \delta, 3}\right) \\
& =E_{w_{1} \delta, 1} * \cdots * E_{w_{t} \delta, 1}+E_{w_{1} \delta, 3} * \cdots * E_{w_{t} \delta, 3}+\text { rest. }
\end{aligned}
$$

Here

$$
\begin{aligned}
& E_{w_{1} \delta, 1} * \cdots * E_{w_{t} \delta, 1}
\end{aligned}
$$

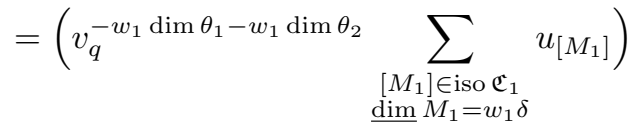

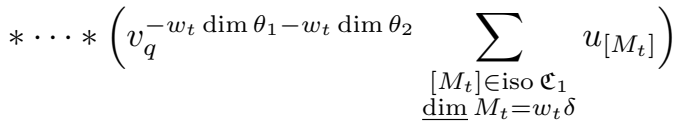

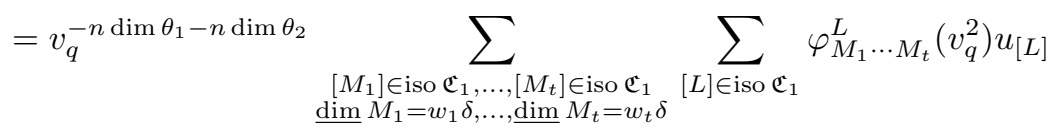

$$
\begin{aligned}
& =\sum_{\substack{\left[M_{1}\right] \in \text { iso }_{\mathfrak{C}_{1}, \ldots,\left[M_{t}\right] \in \text { iso } \mathfrak{C}_{1}} \\
\underline{\operatorname{dim} M_{1}=w_{1} \delta, \ldots, \underline{\operatorname{dim}} M_{t}=w_{t} \delta}}} \sum_{[L] \in \operatorname{iso}} v_{\mathfrak{C}_{1}, \underline{\operatorname{dim}} L=n \delta} v_{q}^{-\operatorname{dim} \operatorname{End} L} \varphi_{M_{1} \cdots M_{t}}^{L}\left(v_{q}^{2}\right)\langle L\rangle .
\end{aligned}
$$

Using formula (8.3) (in $\S 8.1$ ) for the expression of $E_{k \delta, 2}$ in the proof of Lemma 8.2.2, we have

$$
\text { rest }=\sum_{\substack{\left[M_{i_{1}}\right], \ldots,\left[M_{i_{t}}\right] \in \text { iso } \mathfrak{C}_{1} \\ \sum_{j=1}^{t} \underline{\operatorname{dim}} M_{i_{j}}=l \delta, l<n}} \sum_{\substack{[M], w_{\mathbf{c}}^{\prime} ; 0 \neq M \in \mathfrak{C}_{1} \\\left|\left(w_{\mathbf{c}}^{\prime}\right)\right|+l=n}} v_{q}^{-\operatorname{dim} \operatorname{End} M} \varphi_{M_{i_{1}} \cdots M_{i_{t}}}^{M}\left(v_{q}^{2}\right)\langle M\rangle * E_{w_{\mathbf{c}}^{\prime} \delta, 3} .
$$


By applying the above expansion of $E_{w_{\mathbf{c}} \delta}$ to $\left(E_{n \delta}, E_{w_{\mathbf{c}} \delta}\right)$, and by (8.3) (in $\S 8.1$ ) and Lemma 8.3.1, and by applying the genericalization process of $\S 1.4$, we have, in $\mathcal{C}^{*}(\Lambda)_{\mathcal{A}}$

$$
\left(E_{n \delta}, E_{w_{\mathbf{c}} \delta}\right) \equiv\left(E_{n \delta, 3}, E_{w_{\mathbf{c}} \delta, 3}\right)\left(\bmod v^{-1} \mathbb{Q}\left[\left[v^{-1}\right]\right] \cap \mathbb{Q}(v)\right) .
$$

Lemma 8.3.3. Assume $m+n=s+t, m \geq n \geq 0, s \geq t \geq 0$. Then:

(i) If $m \neq s$ or $n \neq t$, then $\left(E_{m \delta}^{\prime} * E_{n \delta}^{\prime}, E_{s \delta}^{\prime} * E_{t \delta}^{\prime}\right)=0$.

(ii) $\left(E_{s \delta}^{\prime} * E_{t \delta}^{\prime}, E_{s \delta}^{\prime} * E_{t \delta}^{\prime}\right)=\left(E_{s \delta}^{\prime}, E_{s \delta}^{\prime}\right)\left(E_{t \delta}^{\prime}, E_{t \delta}^{\prime}\right)$.

(iii) $\left(E_{s \delta}^{\prime} * E_{t \delta}^{\prime}, E_{t \delta}^{\prime} * E_{s \delta}^{\prime}\right)=\left(E_{s \delta}^{\prime}, E_{s \delta}^{\prime}\right)\left(E_{t \delta}^{\prime}, E_{t \delta}^{\prime}\right)$.

(iv) $\left(\left(E_{m \delta}^{\prime}\right)^{* n},\left(E_{m \delta}^{\prime}\right)^{* n}\right)=n !\left(E_{m \delta}^{\prime}, E_{m \delta}^{\prime}\right)^{n}$.

Proof. We remark that if $0 \rightarrow R \rightarrow M \rightarrow I \rightarrow 0$ is exact for a non-zero regular module $R$ and a non-zero preinjective module $I$, then $M$ contains a non-zero preinjective direct summand and no non-zero preprojective summand. Dually, if $0 \rightarrow P \rightarrow N \rightarrow R \rightarrow 0$ is exact for a preprojective module $P$ and a regular module $R$, then $N$ contains a non-zero preprojective direct summand and no nonzero preinjective summand.

Since $\mathcal{R}\left(\mathcal{C}^{*}(\Lambda)\right)_{k \delta}=\mathcal{R}\left(\mathcal{C}^{*}(\Lambda)\right)(k-1)_{k \delta} \oplus \mathbb{Q}(v) E_{k \delta}^{\prime}$, we have

$$
r\left(E_{k \delta}^{\prime}\right)=E_{k \delta}^{\prime} \otimes 1+1 \otimes E_{k \delta}^{\prime}+\sum_{i} a_{k i} \otimes b_{k i}+\sum_{i} x_{k i} *\left\langle I_{k i}\right\rangle \otimes\left\langle P_{k i}\right\rangle * y_{k i},
$$

where $a_{k i}, b_{k i}, x_{k i}, y_{k i} \in \bigcup_{j<k} \mathcal{R}\left(\mathcal{C}^{*}(\Lambda)\right)(j)$ and $I_{k i}$ and $P_{k i}$ are respectively nonzero preinjective modules and non-zero preprojective modules.

We may assume that $s \geq m$; then $n \geq t$. Since

$\left(E_{m \delta}^{\prime} * E_{n \delta}^{\prime}, E_{s \delta}^{\prime} * E_{t \delta}^{\prime}\right)=\left(r\left(E_{m \delta}^{\prime} * E_{n \delta}^{\prime}\right), E_{s \delta}^{\prime} \otimes E_{t \delta}^{\prime}\right)=\left(r\left(E_{m \delta}^{\prime}\right) * r\left(E_{n \delta}^{\prime}\right), E_{s \delta}^{\prime} \otimes E_{t \delta}^{\prime}\right)$,

by the above formula for $r\left(E_{k \delta}^{\prime}\right)$ and the above remark, we have

$$
\begin{aligned}
\left(E_{m \delta}^{\prime} * E_{n \delta}^{\prime}, E_{s \delta}^{\prime} * E_{t \delta}^{\prime}\right)= & \left(\left(E_{m \delta}^{\prime} \otimes 1+1 \otimes E_{m \delta}^{\prime}+\sum_{i} a_{m i} \otimes b_{m i}\right)\right. \\
& \left.*\left(E_{n \delta}^{\prime} \otimes 1+1 \otimes E_{n \delta}^{\prime}+\sum_{i} a_{n i} \otimes b_{n i}\right), E_{s \delta}^{\prime} \otimes E_{t \delta}^{\prime}\right) .
\end{aligned}
$$

If $s>m$, it is easy to compute that the right hand side vanishes. If $s=m>$ $t=n$, it is easy to see that the left side of the above identity is equal to $\left(E_{s \delta}^{\prime}, E_{s \delta}^{\prime}\right)\left(E_{t \delta}^{\prime}, E_{t \delta}^{\prime}\right)$. If $s=t=m=n$, it is equal to $2\left(E_{s \delta}^{\prime}, E_{s \delta}^{\prime}\right)^{2}$. In general, we have $\left(\left(E_{s \delta}^{\prime}\right)^{* l},\left(E_{s \delta}^{\prime}\right)^{* l}\right)=l !\left(E_{s \delta}^{\prime}, E_{s \delta}^{\prime}\right)^{l}$ for $l \geq 0$.

Similarly, we can prove that $\left(E_{s \delta}^{\prime} * E_{t \delta}^{\prime}, E_{t \delta}^{\prime} * E_{s \delta}^{\prime}\right)=\left(E_{s \delta}^{\prime}, E_{s \delta}^{\prime}\right)\left(E_{t \delta}^{\prime}, E_{t \delta}^{\prime}\right)$ for $s>t$. 
Corollary 8.3.4. For $m_{i}, n_{i} \in \mathbb{N}(i=1, \ldots, t)$ satisfying $m_{1}>\cdots>m_{t}$ and $l_{i}, k_{i} \in \mathbb{N}(i=1, \ldots, j)$ satisfying $l_{1}>\cdots>l_{j}$, we have

$$
\begin{aligned}
& \left(\left(E_{m_{1} \delta}^{\prime}\right)^{* n_{1}} * \cdots *\left(E_{m_{t} \delta}^{\prime}\right)^{* n_{t}},\left(E_{l_{1} \delta}^{\prime}\right)^{* k_{1}} * \cdots *\left(E_{l_{j} \delta}^{\prime}\right)^{* k_{j}}\right) \\
& =\left(\left(E_{m_{1} \delta}^{\prime}\right)^{* n_{1}},\left(E_{m_{1} \delta}^{\prime}\right)^{* n_{1}}\right) \cdots\left(\left(E_{m_{t} \delta}^{\prime}\right)^{* n_{t}},\left(E_{m_{t} \delta}^{\prime}\right)^{* n_{t}}\right)
\end{aligned}
$$

if $t=j, m_{i}=l_{i}$, and $n_{i}=k_{i}$ for all $i=1, \ldots, t$, and

$$
\left(\left(E_{m_{1} \delta}^{\prime}\right)^{* n_{1}} * \cdots *\left(E_{m_{t} \delta}^{\prime}\right)^{* n_{t}},\left(E_{l_{1} \delta}^{\prime}\right)^{* k_{1}} * \cdots *\left(E_{l_{j} \delta}^{\prime}\right)^{* k_{j}}\right)=0
$$

otherwise.

For a partition $w=\left(w_{1} \geq \cdots \geq w_{t}\right)$, we define

$$
E_{w \delta}^{\prime}=E_{w_{1} \delta}^{\prime} * \cdots * E_{w_{t} \delta}^{\prime} .
$$

Lemma 8.3.5. Let $\left\{E_{\pi} \mid \pi \in \Pi_{i}^{a}\right\}$ be the $\mathcal{Z}$-basis of $\mathcal{C}^{*}\left(\mathcal{T}_{i}\right)_{\mathcal{Z}}$ defined in $\S 7.3$. Then

$$
\left(E_{\pi} * E_{m \delta}^{\prime}, E_{\pi^{\prime}} * E_{n \delta}^{\prime}\right)=\delta_{m n}\left(E_{\pi}, E_{\pi^{\prime}}\right)\left(E_{m \delta}^{\prime}, E_{n \delta}^{\prime}\right), \quad\left(E_{\pi}, E_{w \delta}^{\prime}\right)=0 .
$$

Proof. We may assume that $m \leq n$. Note that

$$
\begin{aligned}
r\left(E_{\pi}\right)= & E_{\pi} \otimes 1+1 \otimes E_{\pi}+\sum_{\pi_{1}, \pi_{2}} c_{\pi_{1}, \pi_{2}} E_{\pi_{1}} \otimes E_{\pi_{2}} \\
& +\sum_{\pi_{1}, \pi_{2}} d_{\pi_{1}, \pi_{2}} E_{\pi_{1}} *\left\langle I_{\pi_{1}, \pi_{2}}\right\rangle \otimes\left\langle P_{\pi_{1}, \pi_{2}}\right\rangle * E_{\pi_{2}},
\end{aligned}
$$

where $c_{\pi_{1}, \pi_{2}}, d_{\pi_{1}, \pi_{2}} \in \mathcal{Z}, M(\pi), M\left(\pi_{1}\right), M\left(\pi_{2}\right)$ are in $\mathcal{T}_{i}$, and $I_{\pi_{1}, \pi_{2}}$ and $P_{\pi_{1}, \pi_{2}}$ are respectively non-zero preinjective and non-zero preprojective modules. Recall that

$$
r\left(E_{m \delta}^{\prime}\right)=E_{m \delta}^{\prime} \otimes 1+1 \otimes E_{m \delta}^{\prime}+\sum_{i} a_{m i} \otimes b_{m i}+\sum_{i} x_{m i} *\left\langle I_{m i}\right\rangle \otimes\left\langle P_{m i}\right\rangle * y_{m i},
$$

where $a_{m i}, b_{m i}, x_{m i}, y_{m i} \in \bigcup_{j<m} \mathcal{R}\left(\mathcal{C}^{*}(\Lambda)\right)(j)$ and $I_{m i}$ and $P_{m i}$ are respectively non-zero preinjective and non-zero preprojective modules. The same calculation as in the proof of Lemma 8.3.3 tells us that

$$
\left(E_{\pi} * E_{m \delta}^{\prime}, E_{\pi^{\prime}} * E_{n \delta}^{\prime}\right)=\delta_{m n}\left(E_{\pi}, E_{\pi^{\prime}}\right)\left(E_{m \delta}^{\prime}, E_{n \delta}^{\prime}\right) .
$$

The identities now follow from the definition of $E_{w \delta}^{\prime}$.

By Proposition 6.2 , the set $\left\{\left\langle M\left(\mathbf{a}_{\mathbf{c}}\right)\right\rangle * E_{\pi_{1 \mathbf{c}}} * \cdots * E_{\pi_{s \mathbf{c}}} * E_{w_{\mathbf{c}} \delta} *\left\langle M\left(\mathbf{b}_{\mathbf{c}}\right)\right\rangle\right\}$ is a $\mathbb{Q}(v)$-basis of $\mathcal{C}^{*}(\Lambda)_{\mathbb{Q}(v)}$. In the same way, we obtain 
Lemma 8.3.6. The following equalities hold:

(i) $\left(\left\langle M\left(\mathbf{a}_{\mathbf{c}}\right)\right\rangle * E_{\pi_{2 \mathbf{c}}} * \cdots * E_{\pi_{s \mathbf{c}}}, E_{w_{\mathbf{c}^{\prime}} \delta}^{\prime}\right)=0$ for $\mathbf{a}_{\mathbf{c}} \neq 0$ and partition $w_{\mathbf{c}^{\prime}} \neq 0$.

(ii) $\left(E_{\pi}, E_{\pi_{1 \mathbf{c}}} * \cdots * E_{\pi_{s \mathrm{c}}} * E_{w_{\mathbf{c}} \delta}^{\prime}\right)=0$ for $w_{\mathbf{c}} \neq 0$.

(iii) $\left(E_{\pi_{1 \mathrm{c}}} * \cdots * E_{\pi_{s \mathrm{c}}}, E_{\pi_{1 \mathbf{c}^{\prime}}} * \cdots * E_{\pi_{s^{\prime}}} * E_{w_{\mathrm{c}^{\prime}} \delta}^{\prime}\right)=0$ for $w_{\mathbf{c}^{\prime}} \neq 0$.

Based on Lemmas 8.3.5 and 8.3.6, we obtain

Lemma 8.3.7. The following holds:

$$
\left(E_{\pi_{i \mathrm{c}}} * E_{w_{\mathrm{c}} \delta}^{\prime}, E_{\pi_{j \mathrm{c}^{\prime}}} * E_{w_{\mathbf{c}^{\prime}} \delta}^{\prime} \delta=\left(E_{\pi_{i \mathrm{c}}}, E_{\pi_{j \mathrm{c}^{\prime}}}\right)\left(E_{w_{\mathrm{c}} \delta}^{\prime}, E_{w_{\mathbf{c}^{\prime}} \delta}^{\prime}\right), \quad 1 \leq i, j \leq s .\right.
$$

Proof. For a fixed $i$, we have

$$
\begin{aligned}
r\left(E_{\pi_{i \mathrm{c}}}\right)= & E_{\pi_{i \mathrm{c}}} \otimes 1+1 \otimes E_{\pi_{i \mathrm{c}}} \\
& +\sum_{\pi_{1}, \pi_{2}} c_{\pi_{1}, \pi_{2}} E_{\pi_{1}} \otimes E_{\pi_{2}}+\sum_{\pi_{1}, \pi_{2}} d_{\pi_{1}, \pi_{2}} E_{\pi_{1}} *\left\langle I_{\pi_{1}, \pi_{2}}\right\rangle \otimes\left\langle P_{\pi_{1}, \pi_{2}}\right\rangle * E_{\pi_{2}},
\end{aligned}
$$

where $c_{\pi_{1}, \pi_{2}}, d_{\pi_{1}, \pi_{2}} \in \mathcal{Z}, M(\pi), M\left(\pi_{1}\right), M\left(\pi_{2}\right)$ are in $\mathcal{T}_{i}$, and $I_{\pi_{1}, \pi_{2}}$ and $P_{\pi_{1}, \pi_{2}}$ are respectively non-zero preinjective and non-zero preprojective modules. Let

$$
\begin{aligned}
& r^{0}\left(E_{\pi_{i \mathrm{c}}}\right)=E_{\pi_{i \mathrm{c}}} \otimes 1+1 \otimes E_{\pi_{i \mathrm{c}}}+\sum_{\pi_{1}, \pi_{2}} c_{\pi_{1}, \pi_{2}} E_{\pi_{1}} \otimes E_{\pi_{2}}, \\
& r^{1}\left(E_{\pi_{i \mathrm{c}}}\right)=E_{\pi_{i \mathrm{c}}} \otimes 1+1 \otimes E_{\pi_{i \mathrm{c}}} .
\end{aligned}
$$

Also for $w_{\mathbf{c}}=\left(w_{1}, \ldots, w_{t}\right)$,

$$
\begin{aligned}
& r\left(E_{w_{\mathbf{c}} \delta}^{\prime}\right)=r\left(E_{w_{1} \delta}^{\prime}\right) * \cdots * r\left(E_{w_{t} \delta}^{\prime}\right) \\
& =\left(E_{w_{1} \delta}^{\prime} \otimes 1+1 \otimes E_{w_{1} \delta}^{\prime}+\sum_{i} a_{w_{1} i} \otimes b_{w_{1} i}+\sum_{i} x_{w_{1} i} *\left\langle I_{w_{1}}\right\rangle \otimes\left\langle P_{w_{1} i}\right\rangle * y_{w_{1} i}\right) \\
& * \cdots *\left(E_{w_{t} \delta}^{\prime} \otimes 1+1 \otimes E_{w_{t} \delta}^{\prime}+\sum_{i} a_{w_{t} i} \otimes b_{w_{t} i}+\sum_{i} x_{w_{t} i} *\left\langle I_{w_{t}}\right\rangle \otimes\left\langle P_{w_{t} i}\right\rangle * y_{w_{t} i}\right) .
\end{aligned}
$$

Let

$$
\begin{aligned}
r^{0}\left(E_{w_{\mathbf{c}} \delta}^{\prime}\right)= & \left(E_{w_{1} \delta}^{\prime} \otimes 1+1 \otimes E_{w_{1} \delta}^{\prime}+\sum_{i} a_{w_{1} i} \otimes b_{w_{1} i}\right) \\
& * \cdots *\left(E_{w_{t} \delta}^{\prime} \otimes 1+1 \otimes E_{w_{t} \delta}^{\prime}+\sum_{i} a_{w_{t} i} \otimes b_{w_{t} i}\right), \\
r^{1}\left(E_{w_{\mathbf{c}} \delta}^{\prime}\right)= & \left(E_{w_{1} \delta}^{\prime} \otimes 1+1 \otimes E_{w_{1} \delta}^{\prime}\right) * \cdots *\left(E_{w_{t} \delta}^{\prime} \otimes 1+1 \otimes E_{w_{t} \delta}^{\prime}\right),
\end{aligned}
$$

It is clear that

$$
\begin{aligned}
\left(E_{\pi_{i \mathrm{c}}} * E_{w_{\mathrm{c}} \delta}^{\prime}, E_{\pi_{j \mathrm{c}^{\prime}}} * E_{w_{\mathrm{c}^{\prime}} \delta}^{\prime}\right) & =\left(r\left(E_{\pi_{i \mathrm{c}}}\right) * r\left(E_{w_{\mathrm{c}} \delta}^{\prime}\right), E_{\pi_{j \mathrm{c}^{\prime}}} \otimes E_{w_{\mathrm{c}^{\prime}} \delta}^{\prime}\right) \\
& =\left(r^{0}\left(E_{\pi_{i \mathrm{c}}}\right) * r^{0}\left(E_{w_{\mathrm{c}} \delta}^{\prime}\right), E_{\pi_{j \mathrm{c}^{\prime}}} \otimes E_{w_{\mathbf{c}^{\prime}} \delta}^{\prime}\right) .
\end{aligned}
$$


Using Lemmas 8.3.5 and 8.3.6, induction on the length of the partition $w_{\mathbf{c}^{\prime} \delta}$ shows that

$$
\begin{aligned}
& \left(r^{0}\left(E_{\pi_{i \mathrm{c}}}\right) * r^{0}\left(E_{w_{\mathrm{c}} \delta}^{\prime}\right), E_{\pi_{j \mathrm{c}^{\prime}}} \otimes E_{w_{\mathrm{c}^{\prime}} \delta}^{\prime}\right)=\left(r^{1}\left(E_{\pi_{i \mathrm{c}}}\right) * r^{1}\left(E_{w_{\mathrm{c}} \delta}^{\prime} \delta, E_{\pi_{j \mathrm{c}^{\prime}}} \otimes E_{w_{\mathrm{c}^{\prime}} \delta}^{\prime}\right)\right. \\
& =\left(\left(E_{\pi_{i \mathrm{c}}} \otimes 1+1 \otimes E_{\pi_{i \mathrm{c}}}\right) *\left(E_{w_{\mathrm{c} \delta}}^{\prime} \otimes 1+1 \otimes E_{w_{\mathrm{c} \delta}}^{\prime}\right), E_{\pi_{j \mathbf{c}^{\prime}}} \otimes E_{w_{\mathbf{c}^{\prime}} \delta}^{\prime}\right) \\
& =\left(E_{\pi_{i \mathrm{c}}}, E_{\pi_{j \mathrm{c}^{\prime}}}\right)\left(E_{w_{\mathrm{c}} \delta}^{\prime}, E_{w_{\mathrm{c}^{\prime}} \delta}^{\prime}\right) \text {. }
\end{aligned}
$$

Theorem 8.3.8. With the same notation as above,

$$
\begin{aligned}
\left(E_{\pi_{1 \mathrm{c}}} * \cdots * E_{\pi_{s \mathrm{c}}} * E_{w_{\mathrm{c}} \delta}^{\prime},\right. & \left.E_{\pi_{1 \mathrm{c}^{\prime}}} * \cdots * E_{\pi_{s c^{\prime}}} * E_{w_{\mathrm{c}^{\prime}} \delta}^{\prime}\right) \\
& =\left(E_{\pi_{1 \mathrm{c}}}, E_{\pi_{1 \mathrm{c}^{\prime}}}\right)\left(E_{\pi_{s \mathrm{c}}}, E_{\pi_{\mathrm{sc}^{\prime}}}\right) \cdots\left(E_{w_{\mathrm{c}} \delta}^{\prime}, E_{w_{\mathrm{c}^{\prime}} \delta}^{\prime}\right) .
\end{aligned}
$$

\section{$\S 8.4$}

In this subsection, we construct the canonical basis. Let $\mathcal{I} \mathcal{T}$ be the union of the sets of the isomorphism classes of indecomposable objects in the non-homogeneous tubes $\mathcal{T}_{1}, \ldots, \mathcal{T}_{s}$, and $\operatorname{add}(\mathcal{I} \mathcal{T})$ be the set of objects that are isomorphic to direct sums of objects in $\mathcal{I} \mathcal{T}$.

Theorem 8.3.8 and Corollary 8.3.4 imply that $\left(E_{n \delta}^{\prime} * E_{m \delta}^{\prime}-E_{m \delta}^{\prime} * E_{n \delta}^{\prime}, x\right)=0$ for all $x \in \mathcal{R}\left(\mathcal{C}^{*}(\Lambda)\right)$. Thus $E_{n \delta}^{\prime} * E_{m \delta}^{\prime}=E_{m \delta}^{\prime} * E_{n \delta}^{\prime}$ by the non-degeneracy of $(-,-)$ on $\mathcal{R}\left(\mathcal{C}^{*}(\Lambda)\right)$.

Lemma 8.4.1. Assume $\sum_{i=1}^{s} \operatorname{dim} M\left(\pi_{i \mathbf{c}}\right)+\left|w_{\mathbf{c}}\right| \delta=n \delta$. Then:

(i) $\left(E_{n \delta}, E_{\pi_{1 \mathrm{c}}} * \cdots * E_{\pi_{s \mathrm{c}}} * E_{w_{\mathrm{c}} \delta}\right) \in \mathbb{Q}\left[\left[v^{-1}\right]\right] \cap \mathbb{Q}(v)$;

(ii) if $\left|w_{\mathbf{c}}\right| \delta<n \delta$, then $\left(E_{n \delta}, E_{\pi_{1 \mathbf{c}}} * \cdots * E_{\pi_{s \mathbf{c}}} * E_{w_{\mathbf{c}} \delta}\right) \in v^{-1} \mathbb{Q}\left[\left[v^{-1}\right]\right] \cap \mathbb{Q}(v)$;

(iii) $\left(E_{\pi_{1 \mathrm{c}}} * \cdots * E_{\pi_{s \mathrm{c}}} * E_{w_{\mathrm{c}} \delta}, E_{\pi_{1 \mathrm{c}}} * \cdots * E_{\pi_{s \mathrm{c}}} * E_{w_{\mathrm{c}} \delta}\right) \in v^{h}\left(\mathbb{N}+v^{-1} \mathbb{Q}\left[\left[v^{-1}\right]\right] \cap \mathbb{Q}(v)\right)$ for some $h \geq 0$.

Proof. By the proof of Lemma 8.3.2, we have

$$
E_{w_{\mathbf{c}} \delta}=E_{w_{1} \delta, 1} * \cdots * E_{w_{t} \delta, 1}+E_{w_{1} \delta, 3} * \cdots * E_{w_{t} \delta, 3}+\text { rest, }
$$

where, in $\mathcal{H}_{q}^{*}(\Lambda)$,

$$
E_{w_{1} \delta, 1} * \cdots * E_{w_{t} \delta, 1}=\sum_{\substack{\left[M_{1}\right] \in \text { iso } \mathfrak{C}_{1}, \ldots,\left[M_{t}\right] \in \text { iso } \mathfrak{C}_{1} \\ \text { dim } M_{1}=w_{1} \delta, \ldots, \underline{\operatorname{dim}} M_{t}=w_{t} \delta}} \sum_{L] \in \mathfrak{C}_{1}} v_{q}^{-\operatorname{dim} \operatorname{dim} L} \varphi_{M_{1} \cdots M_{t}}^{L}\left(v_{q}^{2}\right)\langle L\rangle
$$

and

$$
\text { rest }=\sum_{\substack{\left[M_{i_{1}}\right], \ldots,\left[M_{i_{t}}\right] \in \text { iso } \mathfrak{C}_{1} \\ \sum_{j=1}^{t} \underline{\operatorname{dim}} M_{i_{j}}=l \delta, l<n}} \sum_{\substack{[M], w_{\mathbf{c}}^{\prime}: 0 \neq M \in \mathfrak{C}_{1} \\ 1 \leq\left|w_{\mathbf{c}}^{\prime}\right|<\left|w_{\mathbf{c}}\right|}} v_{q}^{-\operatorname{dim} \operatorname{End} M} \varphi_{M_{i_{1}} \cdots M_{i_{t}}}^{M}\left(v_{q}^{2}\right)\langle M\rangle * E_{w_{\mathbf{c}}^{\prime} \delta, 3} .
$$


If $M\left(\pi_{i \mathbf{c}}\right)=0$ for all $1 \leq i \leq s$, then both (i) and (iii) are true using the property of complete symmetric functions with respect to the bilinear form $(-,-)$ (see $\S 8.2)$.

Suppose $M\left(\pi_{i \mathbf{c}}\right) \neq 0$ for some $i$. By [DDX],

$$
E_{\pi_{i \mathbf{c}}}=\left\langle M\left(\pi_{i \mathbf{c}}\right)\right\rangle+\sum_{\lambda \in \Pi_{i} \backslash \Pi_{i}^{a}, \lambda \prec \pi_{i \mathbf{c}}} \eta_{\lambda}^{\pi_{i \mathbf{c}}}\langle M(\lambda)\rangle, \quad \text { where } \eta_{\lambda}^{\pi_{i \mathbf{c}}} \in v^{-1} \mathbb{Z}\left[v^{-1}\right] .
$$

Then

$$
E_{\pi_{1 \mathbf{c}}} * \cdots * E_{\pi_{s \mathbf{c}}}=\left\langle\bigoplus_{i=1}^{s} M\left(\pi_{i \mathbf{c}}\right)\right\rangle+\sum_{N \in \text { iso add }\{\mathcal{I} \mathcal{T}\}} \eta_{N}\langle N\rangle,
$$

where $\eta_{N} \in v^{-1} \mathbb{Z}\left[v^{-1}\right]$. Then multiplying them in $\mathcal{H}_{q}^{*}(\Lambda)$ we have

$$
\begin{aligned}
& E_{\pi_{1 \mathbf{c}}} * \cdots * E_{\pi_{s \mathbf{c}}} * E_{w_{1} \delta, 1} * \cdots * E_{w_{t} \delta, 1} \\
& =\sum_{\substack{\left[M_{1}\right] \in \operatorname{iso}_{\mathfrak{C}_{1}, \ldots,\left[M_{t}\right] \in \operatorname{iso} \mathfrak{C}_{1}} \\
\underline{\operatorname{dim} M_{1}=w_{1} \delta, \ldots, \underline{\operatorname{dim}} M_{t}=w_{t} \delta}}} \sum_{[L] \in \mathfrak{C}_{1}} v_{q}^{-\operatorname{dim} \operatorname{End} L} \varphi_{M_{1} \cdots M_{t}}^{L}\left(v_{q}^{2}\right)\left\langle\bigoplus_{i=1}^{s} M\left(\pi_{i \mathbf{c}}\right)\right\rangle *\langle L\rangle
\end{aligned}
$$

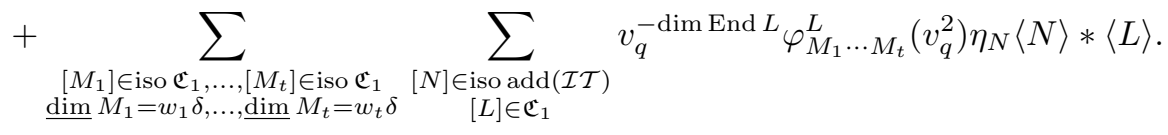

Here,

$$
\begin{aligned}
& \left\langle\bigoplus_{i=1}^{s} M\left(\pi_{i \mathbf{c}}\right)\right\rangle *\langle L\rangle \\
& \quad=\sum_{[U]} v_{q}^{\operatorname{dim} \operatorname{End} \oplus_{i=1}^{s} M\left(\pi_{i \mathbf{c}}\right)+\operatorname{dim} \operatorname{End} L-\operatorname{dim} \operatorname{End} U} \varphi_{\oplus_{i=1}^{s} M\left(\pi_{i \mathbf{c}}\right), L}^{U}\left(v_{q}^{2}\right)\langle U\rangle
\end{aligned}
$$

and

$$
\langle N\rangle *\langle L\rangle=\sum_{[V]} v^{\operatorname{dim} \text { End } N+\operatorname{dim} \text { End } L-\operatorname{dim} \operatorname{End} V} \varphi_{N L}^{V}\left(v^{2}\right)\langle V\rangle .
$$

By Lemma 8.3.1, we know

$$
\begin{aligned}
\operatorname{deg}_{q} \varphi_{\bigoplus_{i=1}^{s}}^{U} M\left(\pi_{i \mathbf{c}}\right), L & \leq \operatorname{dim} \text { End } U-\left(\operatorname{dim} \text { End } L+\operatorname{dim} \operatorname{End} \bigoplus_{i=1}^{s} M\left(\pi_{i \mathbf{c}}\right)\right) \\
\operatorname{deg}_{q} \varphi_{M_{1} \cdots M_{t}}^{L} & \leq \operatorname{dim} \text { End } L-\sum_{i=1}^{t} \operatorname{dim} \text { End } M_{i} \\
\operatorname{deg}_{q} \varphi_{N L}^{V} & \leq \operatorname{dim} \text { End } V-(\operatorname{dim} \operatorname{End} N+\operatorname{dim} \operatorname{End} L)
\end{aligned}
$$


Hence,

$$
\begin{aligned}
& E_{\pi_{1 \mathbf{c}}} * \cdots * E_{\pi_{s \mathbf{c}}} * E_{w_{1} \delta, 1} * \cdots * E_{w_{t} \delta, 1} \\
& =\sum_{0 \neq[U] \in \operatorname{iso} \operatorname{add}(\mathcal{I} \mathcal{T})} v_{q}^{\operatorname{dim} \text { End } U-\operatorname{dim} \text { End } \bigoplus_{i=1}^{s} M\left(\pi_{i \mathbf{c}}\right)-2 \sum_{i} \operatorname{dim} \operatorname{End} M_{i}} f_{U}\left(v_{q}^{-1}\right)\langle U\rangle \\
& +\sum_{0 \neq[V] \in \operatorname{iso} \operatorname{add}(\mathcal{I} \mathcal{T})} v_{q}^{\operatorname{dim} \text { End } V-\operatorname{dim} \operatorname{End} N-2 \sum_{i} \operatorname{dim} \operatorname{End} M_{i}} f_{V}\left(v_{q}^{-1}\right)\langle V\rangle,
\end{aligned}
$$

where $f_{U}\left(v^{-1}\right), f_{V}\left(v^{-1}\right) \in \mathbb{Q}\left[v^{-1}\right]$. In general,

$$
\begin{aligned}
E_{\pi_{1 \mathbf{c}}} * \cdots & * E_{\pi_{s \mathbf{c}}} * E_{w_{\mathbf{c}} \delta} \\
= & \sum_{0 \neq[L] \in \operatorname{iso} \operatorname{add}(\mathcal{I} \mathcal{T})} f_{L}\langle L\rangle+\sum_{\substack{0 \neq[M] \in \operatorname{iso} \operatorname{add}(\mathcal{I} \mathcal{T}) \\
1 \leq\left|w_{\mathbf{c}}^{\prime}\right|<\left|w_{\mathbf{c}}\right|}} f_{M}\langle M\rangle * E_{w_{\mathbf{c}}^{\prime} \delta, 3} \\
& +\left\langle M\left(\pi_{1 \mathbf{c}}\right) \oplus \cdots \oplus M\left(\pi_{s \mathbf{c}}\right)\right\rangle * E_{w_{\mathbf{c}} \delta, 3}+\sum_{[0] \neq[N] \in \operatorname{iso} \operatorname{add}(\mathcal{I} \mathcal{T})} f_{N}\langle N\rangle * E_{w_{\mathbf{c}} \delta, 3},
\end{aligned}
$$

where $v^{-\operatorname{dim} \text { End } L} f_{L}, v^{-\operatorname{dim} \operatorname{End} M} f_{M}, f_{N} \in v^{-1} \mathbb{Q}\left[v^{-1}\right]$.

Using the expressions of $E_{n \delta, 1}, E_{n \delta, 2}, E_{n \delta, 3}$, it is easy to check that (after computation for each $q$ and then taking the genericalization)

$$
\left(E_{n \delta}, E_{\pi_{1 \mathbf{c}}} * \cdots * E_{\pi_{s \mathbf{c}}} * E_{w_{\mathbf{c}} \delta}\right) \in v^{-1} \mathbb{Q}\left[\left[v^{-1}\right]\right] \cap \mathbb{Q}(v) .
$$

Then conclusion (ii) follows.

By Lemma 8.3.2 and the property of complete symmetric functions, we have

$$
\begin{array}{r}
\left(\left\langle M\left(\pi_{1 \mathbf{c}}\right) \oplus \cdots \oplus M\left(\pi_{s \mathbf{c}}\right)\right\rangle * E_{w_{\mathbf{c}} \delta, 3},\left\langle M\left(\pi_{1 \mathbf{c}}\right) \oplus \cdots \oplus M\left(\pi_{s \mathbf{c}}\right)\right\rangle * E_{w_{\mathbf{c}} \delta, 3}\right) \\
\in \mathbb{N}^{*}+v^{-1} \mathbb{Q}\left[\left[v^{-1}\right]\right] \cap \mathbb{Q}(v) .
\end{array}
$$

Thus

$$
\left(E_{\pi_{1 \mathbf{c}}} * \cdots * E_{\pi_{s \mathbf{c}}} * E_{w_{\mathbf{c}} \delta}, E_{\pi_{1 \mathbf{c}}} * \cdots * E_{\pi_{s \mathbf{c}}} * E_{w_{\mathbf{c}} \delta}\right) \in v^{h}\left(\mathbb{N}+v^{-1} \mathbb{Q}\left[\left[v^{-1}\right]\right] \cap \mathbb{Q}(v)\right)
$$

for some $h \geq 0$. Thus (iii) is proved.

Using [L5], the lattice $\mathcal{L}^{\prime}$ defined in $\S 7.2$ is the $\mathbb{Q}\left[v^{-1}\right]$-submodule of $\mathcal{C}^{*}(\Lambda)_{\mathcal{A}}$ characterized by

$$
\mathcal{L}^{\prime}=\left\{x \in \mathcal{C}^{*}(\Lambda) \mid(x, x) \in \mathbb{Q}\left[\left[v^{-1}\right]\right] \cap \mathbb{Q}(v)\right\} .
$$

Lemma 8.4.2. We have $E_{n \delta}^{\prime} \in \mathcal{L}^{\prime}$ and $\left(E_{n \delta}^{\prime}, E_{n \delta}^{\prime}\right) \equiv 1 / n\left(\bmod v^{-1} \mathbb{Q}\left[\left[v^{-1}\right]\right] \cap \mathbb{Q}(v)\right)$. 
Proof. We know that $\left\{E_{\pi_{i \mathrm{c}}} \mid \underline{\operatorname{dim}} M\left(\pi_{i \mathrm{c}}\right)=\delta, 1 \leq i \leq s\right\}$ is a basis of $\mathcal{R}^{a}\left(\mathcal{C}^{*}(\Lambda)\right)_{\delta}$. Via Schmidt orthogonalization, we define $E_{\pi_{i c}}^{\prime}$ to be the orthogonal element corresponding to $E_{\pi_{i c}}$ for $i=1, \ldots, s$. We use induction on $n$.

For $n=1$, it is easy to see that $\left(E_{\pi}^{\prime}, E_{\pi}^{\prime}\right) \equiv 1$ and $\left(E_{\delta}, E_{\pi}^{\prime}\right) \equiv 0$ $\left(\bmod v^{-1} \mathbb{Q}\left[\left[v^{-1}\right]\right] \cap \mathbb{Q}(v)\right)$. Thus

$$
E_{\delta}^{\prime}=E_{\delta}-\sum_{\pi} \frac{\left(E_{\delta}, E_{\pi}^{\prime}\right)}{\left(E_{\pi}^{\prime}, E_{\pi}^{\prime}\right)} E_{\pi}^{\prime}
$$

Hence, $\left.\left(E_{\delta}^{\prime}, E_{\delta}^{\prime}\right) \equiv 1\left(\bmod v^{-1} \mathbb{Q}\left[\left[v^{-1}\right]\right] \cap \mathbb{Q}(v)\right)\right)$.

Now suppose $\left(E_{m \delta}^{\prime}, E_{m \delta}^{\prime}\right) \equiv 1 / m\left(\bmod v^{-1} \mathbb{Q}\left[\left[v^{-1}\right]\right] \cap \mathbb{Q}(v)\right)$ for all $m<n$. By the definition of $E_{n \delta}^{\prime}$, the set

$$
\left\{E_{n \delta}^{\prime}\right\} \cup\left\{E_{\pi_{1 \mathbf{c}}} * \cdots * E_{\pi_{s \mathbf{c}}} * E_{w_{\mathbf{c}} \delta}^{\prime}\left|\sum_{1 \leq i \leq s} \underline{\operatorname{dim}} M\left(\pi_{i \mathbf{c}}\right)+\right| w_{\mathbf{c}} \mid \delta=n \delta \text { and }\left|w_{\mathbf{c}}\right|<n\right\}
$$

is a basis of $\mathcal{R}\left(\mathcal{C}^{*}(\Lambda)\right)_{n \delta}$.

By Theorem 8.3.8 and the induction hypothesis, $\left\{E_{\pi_{1 \mathrm{c}}} * \cdots * E_{\pi_{s \mathrm{c}}} * E_{w_{\mathrm{c}} \delta}^{\prime}, E_{n \delta}\right\}$ $\subset \mathcal{L}^{\prime}$. Similar to Lemma 8.4.1, we get $\left(E_{n \delta}, E_{\pi_{1 \mathrm{c}}} * \cdots * E_{\pi_{s \mathrm{c}}} * E_{w_{\mathrm{c}} \delta}^{\prime}\right) \in v^{-1} \mathbb{Q}\left[\left[v^{-1}\right]\right] \cap$ $\mathbb{Q}(v)$ if there exists $i$ such that $M\left(\pi_{i \mathbf{c}}\right) \neq 0$. Thus

$$
E_{n \delta}^{\prime} \equiv E_{n \delta}-\sum_{w_{\mathbf{c}} \vdash n, w_{\mathbf{c}} \neq(n)} \frac{\left(E_{n \delta}, E_{w_{\mathbf{c}} \delta}^{\prime}\right)}{\left(E_{w_{\mathbf{c}} \delta}^{\prime}, E_{w_{\mathbf{c}} \delta}^{\prime} \delta\right.} E_{w_{\mathbf{c}} \delta}^{\prime}\left(\bmod v^{-1} \mathcal{L}^{\prime}\right) .
$$

First of all, $\left(E_{n \delta}^{\prime}, E_{n \delta}^{\prime}\right)=\left(E_{n \delta}, E_{n \delta}^{\prime}\right)$ since $\left(E_{n \delta}^{\prime}, E_{w_{\mathbf{c}} \delta}^{\prime}\right)=0$ if $w_{\mathbf{c}} \neq(n)$. We now claim that

$$
\left(E_{n \delta}, E_{w_{\mathrm{c}} \delta}^{\prime}\right)=\left(E_{w_{1}}^{\prime}, E_{w_{1}}^{\prime}\right)^{k_{1}} \cdots\left(E_{w_{t}}^{\prime}, E_{w_{t}}^{\prime}\right)^{k_{t}}
$$

if $w_{\mathbf{c}}=\left(w_{1}^{k_{1}}, \ldots, w_{t}^{k_{t}}\right), w_{1}>\cdots>w_{t}$. Note that

$$
r\left(E_{n \delta}\right)=\sum_{0 \leq i \leq n} E_{i \delta} \otimes E_{(n-i) \delta}+\text { rest. }
$$

Let $r^{0}\left(E_{n \delta}\right)=\sum_{0 \leq i \leq n} E_{i \delta} \otimes E_{(n-i) \delta}$ and $w_{\mathbf{c}}^{\prime}=\left(w_{1}^{k_{1}-1}, w_{2}^{k_{2}}, \ldots, w_{t}^{k_{t}}\right)$. Then

$$
\left(E_{n \delta}, E_{w_{\mathrm{c}} \delta}^{\prime}\right)=\left(r\left(E_{n \delta}\right), E_{w_{1} \delta}^{\prime} \otimes E_{w_{\mathrm{c}}^{\prime} \delta}^{\prime}\right)=\left(r^{0}\left(E_{n \delta}\right), E_{w_{1} \delta}^{\prime} \otimes E_{w_{\mathrm{c}}^{\prime} \delta}^{\prime}\right) .
$$

Based on the definition of $E_{w_{1} \delta}^{\prime}$ and Theorem 8.3.8, we have

$$
\left(E_{n \delta}, E_{w_{\mathbf{c}} \delta}^{\prime}\right)=\left(E_{w_{1} \delta}, E_{w_{1} \delta}^{\prime}\right)\left(E_{\left(n-w_{1}\right) \delta}, E_{w_{\mathbf{c}}^{\prime} \delta}^{\prime}\right) .
$$

Now (8.6) follows by induction on the length of the partition $w_{\mathbf{c}}$. 
On the other hand, Lemma 8.3.3(iv) and Corollary 8.3.4 imply

$$
\left(E_{w_{\mathbf{c}} \delta}^{\prime}, E_{w_{\mathbf{c}} \delta}^{\prime}\right)=k_{1} !\left(E_{w_{1} \delta}^{\prime}, E_{w_{1} \delta}^{\prime}\right)^{k_{1}} \cdots k_{t} !\left(E_{w_{t} \delta}^{\prime}, E_{w_{t} \delta}^{\prime}\right)^{k_{t}} .
$$

Using (8.7) and (8.6) we have

$$
\begin{aligned}
& \left(E_{n \delta}^{\prime}, E_{n \delta}^{\prime}\right) \equiv\left(E_{n \delta}, E_{n \delta}\right)-\sum_{w_{\mathbf{c}} \vdash n, w_{\mathbf{c}} \neq(n)} \frac{\left(E_{n \delta}, E_{w_{\mathbf{c}} \delta}^{\prime}\right)^{2}}{\left(E_{w_{\mathbf{c}} \delta}^{\prime}, E_{w_{\mathbf{c}} \delta}^{\prime}\right)} \\
& \equiv\left(E_{n \delta}, E_{n \delta}\right)-\sum_{(n) \neq\left(1^{r_{1}} 2^{r_{2}} \ldots\right) \vdash n} \frac{\prod_{i \geq 1}\left(E_{i \delta}^{\prime}, E_{i \delta}^{\prime}\right)^{r_{i}}}{\prod_{i \geq 1} r_{i} !} \\
& \equiv 1-\sum_{(n) \neq\left(1^{r_{1}} 2^{r_{2}} \ldots\right) \vdash n} \frac{1}{\prod_{i \geq 1} r_{i} ! i^{r_{i}}} \quad \text { (by the induction hypothesis ) } \\
& =1-\sum_{\left(1^{r_{1}} 2^{r_{2} \ldots}\right) \vdash n} \frac{1}{\prod_{i \geq 1} r_{i} ! i^{r_{i}}}+\frac{1}{n}=\frac{1}{n} \text {. }
\end{aligned}
$$

We have used the identity $n !=\sum_{\left(1^{r_{1}} 2^{r_{2}} \ldots\right) \vdash n} \frac{n !}{\prod_{i \geq 1} r_{i} ! i^{r_{i}}}$ in the last equality.

Let $P_{n \delta}=n E_{n \delta}^{\prime}$. For a partition $w_{\mathbf{c}}=\left(1^{r_{1}} 2^{r_{2}} \cdots t^{r_{t}}\right)$, let $z_{w_{\mathbf{c}}}=\prod_{i \geq 1} i^{r_{i}} r_{i}$ ! and $P_{w_{\mathbf{c}} \delta}=P_{1 \delta}^{* r_{1}} * \cdots * P_{t \delta}^{* r_{t}}$.

Corollary 8.4.3. Let $w_{\mathbf{c}}=\left(1^{r_{1}} 2^{r_{2}} \ldots\right), w_{\mathbf{c}^{\prime}}=\left(1^{r_{1}^{\prime}} 2^{r_{2}^{\prime}} \ldots\right)$ be partitions. Then

$$
\begin{gathered}
\left(E_{\pi_{1 \mathbf{c}}} * \cdots * E_{\pi_{s \mathbf{c}}} * E_{w_{\mathbf{c}} \delta}^{\prime}, E_{\pi_{1 \mathbf{c}^{\prime}}} * \cdots * E_{\pi_{s \mathbf{c}^{\prime}}} * E_{w_{\mathbf{c}^{\prime}} \delta}^{\prime}\right) \\
\equiv \delta_{\pi_{1 \mathbf{c}}, \pi_{1 \mathbf{c}^{\prime}}} \cdots \delta_{\pi_{1 \mathbf{c}}, \pi_{1 \mathbf{c}^{\prime}}} \delta_{w_{\mathbf{c}}, w_{\mathbf{c}^{\prime}}} \prod_{i} r_{i} !\left(E_{i \delta}^{\prime}, E_{i \delta}^{\prime}\right)^{r_{i}}\left(\bmod v^{-1} \mathbb{Q}\left[\left[v^{-1}\right]\right] \cap \mathbb{Q}(v)\right) \\
\left(P_{n \delta}, P_{n \delta}\right) \equiv n\left(\bmod v^{-1} \mathbb{Q}\left[\left[v^{-1}\right]\right] \cap \mathbb{Q}(v)\right) \\
\left(P_{w_{\mathbf{c}} \delta}, P_{w_{\mathbf{c}} \delta}\right) \equiv \delta_{w_{\mathbf{c}} w_{\mathbf{c}^{\prime}}} z_{w_{\mathbf{c}}}\left(\bmod v^{-1} \mathbb{Q}\left[\left[v^{-1}\right]\right] \cap \mathbb{Q}(v)\right) .
\end{gathered}
$$

By this property of $P_{w_{\mathbf{c}} \delta}$, it is easy to see that $P_{w_{\mathbf{c}} \delta}$ corresponds to Newton symmetric functions (i.e., power sum symmetric functions). Let $S_{w_{\mathbf{c}} \delta}$ be the Schur functions corresponding to $P_{w_{\mathbf{c}} \delta}$, and $e^{\mathbf{c}}=\left\langle M\left(\mathbf{a}_{\mathbf{c}}\right)\right\rangle * E_{\pi_{1 \mathbf{c}}} * \cdots * E_{\pi_{s \mathbf{c}}} * S_{w_{\mathbf{c}}} \delta *\left\langle M\left(\mathbf{b}_{\mathbf{c}}\right)\right\rangle$ for $\mathbf{c} \in \mathcal{M}$.

By Theorem 8.3.8, Lemma 8.4.2, Corollary 8.4.3, and the Nakayama Lemma, we have the following corollary:

Corollary 8.4.4. $\left\{e^{\mathbf{c}} \mid \mathbf{c} \in \mathcal{M}\right\}$ is an almost orthonormal basis of $\mathcal{L}^{\prime}$, that is, $\left(e^{\mathbf{c}}, e^{\mathbf{c}^{\prime}}\right) \in \delta_{\mathbf{c}, \mathbf{c}^{\prime}}+v^{-1} \mathbb{Q}\left[\left[v^{-1}\right]\right] \cap \mathbb{Q}(v)$ for $\mathbf{c}, \mathbf{c}^{\prime} \in \mathcal{M}$.

In $\S 8.1$, we have defined the constructible set

$$
\mathcal{O}_{\mathbf{c}}=\mathcal{O}_{M\left(\mathbf{a}_{\mathbf{c}}\right)} \star \mathcal{O}_{M_{\pi_{1 \mathbf{c}}}} \star \cdots \star \mathcal{O}_{M_{\pi_{s \mathbf{c}}}} \star \mathcal{N}_{w_{\mathbf{c}} \delta} \star \mathcal{O}_{M\left(\mathbf{b}_{\mathbf{c}}\right)}
$$


for any $\mathbf{c} \in \mathcal{M}$. Now we define a new partial order $\prec$ for those $e^{\mathbf{c}}, \mathbf{c} \in \mathcal{M}$, with the same dimension vector as follows:

$$
e^{\mathbf{c}} \prec e^{\mathbf{c}^{\prime}} \text { if either } \operatorname{dim} \mathcal{O}_{\mathbf{c}}<\operatorname{dim} \mathcal{O}_{\mathbf{c}^{\prime}} \text {, or } \operatorname{dim} \mathcal{O}_{\mathbf{c}}=\operatorname{dim} \mathcal{O}_{\mathbf{c}^{\prime}} \text { but } w_{\mathbf{c}}>w_{\mathbf{c}^{\prime}} \text {. }
$$

Based on the definition of $E_{n \delta}^{\prime}$, we have

$$
\begin{aligned}
E_{n \delta}^{\prime}= & E_{n \delta}-\sum_{w_{\mathbf{c}} \vdash n, w_{\mathbf{c}} \neq(n)} \frac{\left(E_{n \delta}, E_{w_{\mathbf{c}} \delta}^{\prime}\right)}{\left(E_{w_{\mathbf{c}} \delta}^{\prime}, E_{w_{\mathbf{c}} \delta}^{\prime}\right)} E_{w_{\mathbf{c}} \delta}^{\prime} \\
& +\sum_{\operatorname{dim} \mathcal{O}_{\mathbf{c}^{\prime}}<\operatorname{dim} \mathcal{O}_{n \delta}} a_{n \delta, \mathbf{c}^{\prime}} E_{\pi_{1 \mathbf{c}^{\prime}}} * \cdots * E_{\pi_{s \mathbf{c}^{\prime}}} * S_{w_{\mathbf{c}^{\prime}} \delta},
\end{aligned}
$$

where $a_{n \delta, \mathbf{c}^{\prime}} \in \mathbb{Q}(v)$. In fact, by Corollary 8.4.4, we have $a_{n \delta, \mathbf{c}^{\prime}} \in \mathbb{Q}\left[\left[v^{-1}\right]\right] \cap \mathbb{Q}(v)$. Thus

$$
\begin{aligned}
E_{n \delta}= & E_{n \delta}^{\prime}+\sum_{w_{\mathbf{c}} \vdash n, w_{\mathbf{c}} \neq(n)} \frac{\left(E_{n \delta}, E_{w_{\mathbf{c}} \delta}^{\prime}\right)}{\left(E_{w_{\mathbf{c}} \delta}^{\prime}, E_{w_{\mathbf{c}} \delta}^{\prime}\right)} E_{w_{\mathbf{c}} \delta}^{\prime} \\
& +\sum_{\operatorname{dim} \mathcal{O}_{\mathbf{c}^{\prime}}<\operatorname{dim} \mathcal{O}_{n \delta}} a_{n \delta, \mathbf{c}^{\prime}} E_{\pi_{1 \mathbf{c}^{\prime}}} * \cdots * E_{\pi_{s \mathbf{c}^{\prime}}} * S_{w_{\mathbf{c}^{\prime}} \delta} \\
E_{n \delta}= & \frac{1}{n} P_{n \delta}+\sum_{w_{\mathbf{c}} \vdash n, w_{\mathbf{c}} \neq(n)} \frac{1}{z_{w_{\mathbf{c}}}} P_{w_{\mathbf{c}} \delta} \\
& +\sum_{\operatorname{dim} \mathcal{O}_{\mathbf{c}^{\prime}}<\operatorname{dim} \mathcal{O}_{n \delta}} a_{n \delta, \mathbf{c}^{\prime}} E_{\pi_{1 \mathbf{c}^{\prime}}} * \cdots * E_{\pi_{s \mathbf{c}^{\prime}}} * S_{w_{\mathbf{c}^{\prime}} \delta}
\end{aligned}
$$

Let $H_{n \delta}$ be the $n$th complete symmetric function corresponding to $P_{n \delta}$. From [M, p. 25], we have

$$
E_{n \delta}=H_{n \delta}+\sum_{\operatorname{dim} \mathcal{O}_{\mathbf{c}^{\prime}}<\operatorname{dim} \mathcal{O}_{n \delta}} a_{n \delta, \mathbf{c}^{\prime}} E_{\pi_{1 \mathbf{c}^{\prime}}} * \cdots * E_{\pi_{s \mathbf{c}^{\prime}}} * S_{w_{\mathbf{c}^{\prime}} \delta} .
$$

Let $w_{\mathbf{c}}$ be a partition of $n$. According to Lemma 2.2 and the above formula, we have

$$
E_{w_{\mathbf{c}} \delta}=H_{w_{\mathbf{c}} \delta}+\sum_{\operatorname{dim} \mathcal{O}_{\mathbf{c}^{\prime}}<\operatorname{dim} \mathcal{O}_{n \delta}} a_{n \delta, \mathbf{c}^{\prime}} E_{\pi_{1 \mathbf{c}^{\prime}}} * \cdots * E_{\pi_{s \mathbf{c}^{\prime}}} * S_{w_{\mathbf{c}^{\prime}} \delta} .
$$

There is a monomial $\mathfrak{m}_{w_{\mathbf{c}} \delta}$ in the divided powers of $u_{\left[S_{i}\right]}(i \in I)$ in Proposition 7.4 corresponding to $E_{w_{\mathbf{c}} \delta}$ such that

$$
\begin{aligned}
& \mathfrak{m}_{w_{\mathbf{c}} \delta}=H_{w_{\mathbf{c}} \delta}+\sum_{\operatorname{dim} \mathcal{O}_{\mathbf{c}^{\prime}}<\operatorname{dim} \mathcal{O}_{n \delta}} b_{n \delta, \mathbf{c}^{\prime}} E_{\pi_{1 \mathbf{c}^{\prime}}} * S_{w_{\mathbf{c}^{\prime}} \delta} \\
& +\sum_{\substack{\operatorname{dim} \mathcal{O}_{\mathbf{c}^{\prime}}<\operatorname{dim} \mathcal{O}_{n \delta} \\
M\left(\mathbf{a}_{\mathbf{c}^{\prime}} \neq 0 \text { or } M\left(\mathbf{b}_{\mathbf{c}^{\prime}}\right) \neq 0\right.}} c_{n \delta, \mathbf{c}^{\prime}} e^{\mathbf{c}^{\prime}}
\end{aligned}
$$




$$
\begin{aligned}
= & S_{w_{\mathbf{c}} \delta}+\sum_{w_{\mathbf{c}^{\prime \prime}}>w_{\mathbf{c}}} K_{w_{\mathbf{c}^{\prime \prime}} w_{\mathbf{c}}} S_{w_{\mathbf{c}^{\prime \prime}} \delta}+\sum_{\operatorname{dim} \mathcal{O}_{\mathbf{c}^{\prime}}<\operatorname{dim} \mathcal{O}_{n \delta}} b_{n \delta, \mathbf{c}^{\prime}} E_{\pi_{1 \mathbf{c}^{\prime}}} * \cdots * E_{\pi_{s^{\prime}}} * S_{w_{\mathbf{c}^{\prime}} \delta} \\
& +\sum_{\substack{\operatorname{dim} \mathcal{O}_{\mathbf{c}^{\prime}}<\operatorname{dim} \mathcal{O}_{n \delta} \\
M\left(\mathbf{a}_{\mathbf{c}^{\prime}}\right) \neq 0 \text { or } M\left(\mathbf{b}_{\mathbf{c}^{\prime}}\right) \neq 0}} c_{n \delta, \mathbf{c}^{\prime}} e^{\mathbf{c}^{\prime},},
\end{aligned}
$$

where $K_{\mu \lambda}$ are Kostka numbers and $b_{n \delta, \mathbf{c}^{\prime}}, c_{n \delta, \mathbf{c}^{\prime}} \in \mathbb{Q}(v)$. Furthermore, for $\mathbf{c} \in \mathcal{M}$ and the monomials $\mathfrak{m}_{\mathbf{c}}$ given in Proposition 7.4, we have

$$
\mathfrak{m}_{\mathbf{c}}=e^{\mathbf{c}}+\sum_{e^{\mathbf{c}^{\prime}} \prec e^{\mathbf{c}}} a_{\mathbf{c}^{\prime} \mathbf{c}} e^{\mathbf{c}^{\prime}},
$$

where $a_{\mathbf{c}^{\prime} \mathbf{c}} \in \mathbb{Q}(v)$. Proposition 7.4 and the above formulae tell us that the transition matrix between $\left\{E^{\mathbf{c}} \mid \mathbf{c} \in \mathcal{M}\right\}$ and $\left\{e^{\mathbf{c}} \mid \mathbf{c} \in \mathcal{M}\right\}$ is triangular with diagonal entries equal to 1 , and $\left\{E^{\mathbf{c}} \mid \mathbf{c} \in \mathcal{M}\right\}$ is an $\mathcal{A}$-basis of $\mathcal{C}^{*}(\Lambda)_{\mathcal{A}},\left\{e^{\mathbf{c}} \mid \mathbf{c} \in \mathcal{M}\right\} \subset \mathcal{L}^{\prime}$ and $\left\{\mathfrak{m}_{\mathbf{c}} \mid \mathbf{c} \in \mathcal{M}\right\} \subset \mathcal{C}^{*}(\Lambda)_{\mathcal{A}}$. Thus the constants $a_{\mathbf{c}^{\prime} \mathbf{c}}$ in the above formulae must lie in $\mathcal{A}$.

By applying the same argument as in Section 7 to $\left\{e^{\mathbf{c}} \mid \mathbf{c} \in \mathcal{M}\right\}$, we obtain an $\mathcal{A}$-basis of $\mathcal{C}^{*}(\Lambda)_{\mathcal{A}}$ which is denoted by $\left\{\mathcal{E}^{\mathbf{c}} \mid \mathbf{c} \in \mathcal{M}\right\}$ satisfying

$$
\mathcal{E}^{\prime \mathbf{c}}=\sum_{\mathbf{c}^{\prime} \in \mathcal{M}} \zeta_{\mathbf{c}^{\prime}}^{\mathbf{c}} e^{\mathbf{c}^{\prime}} \quad \text { for any } \mathbf{c} \in \mathcal{M}
$$

where $\zeta_{\mathbf{c}}^{\mathbf{c}}=1$ and $\zeta_{\mathbf{c}^{\prime}}^{\mathbf{c}} \in v^{-1} \mathbb{Q}\left[v^{-1}\right]$ if $e^{\mathbf{c}^{\prime}} \prec e^{\mathbf{c}}$.

Finally, we have the following theorem:

Theorem 8.4.5 The set $\left\{\mathcal{E}^{\prime \mathbf{c}} \mid \mathbf{c} \in \mathcal{M}\right\} \subset \mathcal{L}^{\prime}$ is an $\mathcal{A}$-basis of $\mathcal{C}^{*}(\Lambda)_{\mathcal{A}}$ which is characterized by the following three properties:

(i) $\overline{\mathcal{E}^{\prime \mathbf{c}}}=\mathcal{E}^{\prime \mathbf{c}}$ for all $\mathbf{c} \in \mathcal{M}$.

(ii) $\pi\left(\mathcal{E}^{\prime \mathbf{c}}\right)=\pi\left(\mathcal{E}^{\mathbf{c}}\right)$, where $\pi: \mathcal{L}^{\prime} \rightarrow \mathcal{L}^{\prime} / v^{-1} \mathcal{L}^{\prime}$ is the canonical projection.

(iii) $\left(\mathcal{E}^{\mathbf{c}}, \mathcal{E}^{\prime \mathbf{c}^{\prime}}\right) \equiv \delta_{\mathbf{c c}}\left(\bmod v^{-1} \mathbb{Q}\left[\left[v^{-1}\right]\right] \cap \mathbb{Q}(v)\right)$.

According to Lusztig [L5], we have obtained the signed canonical basis $\left(\mathcal{E}^{\prime \mathbf{c}}\right)$ of $\mathcal{L}^{\prime}$. From the above formulae, we have the relations

$$
\mathfrak{m}_{\mathbf{c}}=\mathcal{E}^{\prime \mathbf{c}}+\sum_{e^{\mathbf{c}^{\prime}} \prec e^{\mathbf{c}}} d_{\mathbf{c}^{\prime} \mathbf{c}} \mathcal{E}^{\prime \mathbf{c}^{\prime}}, \quad \text { where } d_{\mathbf{c}^{\prime} \mathbf{c}} \in \mathcal{A} .
$$

By the total positivity of the canonical basis, we have

Theorem 8.4.6. The set $\left\{\mathcal{E}^{\prime \mathbf{c}} \mid \mathbf{c} \in \mathcal{M}\right\}$ is the canonical basis of $\mathcal{L}^{\prime}$ in the sense of Lusztig.

This answers a question raised by Nakajima in $[\mathrm{N}]$. 


\section{§9. Appendix: Canonical basis for the Kronecker quiver}

For the Kronecker quiver, one could follow the same argument as in Section 8 to construct the canonical basis. Since the Kronecker quiver has the strong representation-directed property, we will make the computation explicit following the approach in [L1, 7.8-7.11]. We remark that although integral bases are constructed in $[\mathrm{C}]$ and $[\mathrm{Z} 2]$, their relation to the canonical basis presented here is new.

\section{$\S 9.1$}

In this section, we work in $\mathcal{C}^{*}=\mathcal{C}^{*}(\Lambda)_{\mathcal{Z}}$. Recall from $\S 5.1$ that $\Phi^{+}$is the positive root system of $\hat{s l}_{2}$. For each positive root $\alpha$, there is exactly one absolutely indecomposable module in $\bmod \Lambda$ with dimension vector $\alpha$ since the imaginary roots have multiplicity 1. A function $\mathbf{c}: \Phi^{+} \rightarrow \mathbb{N}$ is called support-finite if $\mathbf{c}(\alpha) \neq 0$ only for finitely many $\alpha \in \Phi^{+}$. Let $\mathbb{N}_{f}^{\Phi^{+}}$be the set of all support-finite $\mathbb{N}$-valued functions. We will use the order in $\Phi^{+}$given in $\S 5.1$.

For $\mathbf{c} \in \mathbb{N}_{f}^{\Phi^{+}}$, if $\left\{\alpha \in \Phi^{+} \mid \mathbf{c}(\alpha) \neq 0\right\}=\left\{\beta_{1}<\cdots<\beta_{k}\right\}$, we set

$$
E^{\mathbf{c}}=E_{\beta_{1}}^{\left(* \mathbf{c}\left(\beta_{1}\right)\right)} * \cdots * E_{\beta_{k}}^{\left(* \mathbf{c}\left(\beta_{k}\right)\right)},
$$

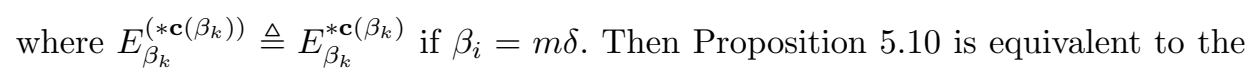
statement that

$$
\begin{aligned}
\left\{E^{\mathbf{c}} \mid \mathbf{c}\right. & \left.\in \mathbb{N}_{f}^{\Phi^{+}}\right\} \\
& =\left\{\langle P(\mathbf{c})\rangle * E_{w_{\mathbf{c}} \delta} *\langle I(\mathbf{c})\rangle \mid P(\mathbf{c}) \in \mathcal{P}_{\text {prep }}, w \in \mathbf{P}(n), I(\mathbf{c}) \in \mathcal{P}_{\text {prei }}, n \in \mathbb{N}\right\}
\end{aligned}
$$

is a $\mathcal{Z}$-basis of $\mathcal{C}^{*}$.

Let $E^{\mathbf{c}}=\langle P(\mathbf{c})\rangle * E_{w_{\mathbf{c}} \delta} *\langle I(\mathbf{c})\rangle$ be as in $\S 7.1$. We define the variety

$$
\mathcal{O}_{\mathbf{c}}:=\mathcal{O}_{P(\mathbf{c}), w_{\mathbf{c}}, I(\mathbf{c})}=\mathcal{O}_{P(\mathbf{c})} \star \mathcal{N}_{w_{\mathbf{c}} \delta} \star \mathcal{O}_{I(\mathbf{c})}
$$

for any $\mathbf{c} \in \mathcal{M}$, where $\mathcal{N}_{w_{\mathbf{c}} \delta}=\mathcal{N}_{w_{1} \delta} \star \cdots \star \mathcal{N}_{w_{t} \delta}$ if $w_{\mathbf{c}}=\left(w_{1}, \ldots, w_{t}\right)$ and $\mathcal{N}_{w_{i} \delta}$ is the union of the orbits of regular modules with dimension vector $w_{i} \delta$.

For $\mathbf{d}=\left(d_{1}, d_{2}\right) \in \mathbb{N}^{2}$, we denote

$$
E(\mathbf{d})=E_{2}^{\left(* d_{2}\right)} * E_{1}^{\left(* d_{1}\right)} .
$$

Note that $\Phi^{+} \subset \mathbb{N}^{2}$. Similarly we define

$$
E(\mathbf{c})=E\left(\mathbf{c}\left(\beta_{1}\right) \beta_{1}\right) * \cdots * E\left(\mathbf{c}\left(\beta_{k}\right) \beta_{k}\right) .
$$

Note that $E(\mathbf{c}) \in \mathcal{C}^{*}$ since it is a monomial in the Chevalley generators $E_{1}$ and $E_{2}$ in the form of divided powers. Moreover, by definition, $\overline{E(\mathbf{d})}=E(\mathbf{d})$. Thus $\overline{E(\mathbf{c})}=E(\mathbf{c})$. 


\section{$\S 9.2$}

The rest of this section is devoted to giving a triangular relation between the PBW basis and the monomial basis.

Lemma 9.2. For any $\mathbf{c} \in \mathbb{N}_{f}^{\Phi^{+}}$and any real root $\beta \in \Phi^{+}$, in $\mathcal{C}^{*}$ we have

$$
E(\mathbf{c}(\beta) \beta)=\left\langle u_{\mathbf{c}(\beta) \beta}\right\rangle+\sum_{\substack{\mathbf{c}^{\prime} \in \mathbb{N}_{f}^{\Phi+} \\ \operatorname{dim} \mathcal{O}_{\mathbf{c}^{\prime}}<\operatorname{dim} \mathcal{O}_{\mathbf{c}(\beta) \beta}}} v^{\lambda\left(\mathbf{c}^{\prime}\right)} E^{\mathbf{c}^{\prime}}, \quad \text { where } \lambda\left(\mathbf{c}^{\prime}\right) \in \mathbb{Z} .
$$

Proof. Let $\mathbf{c}(\beta) \beta=(m, n)$. In $\mathcal{H}_{q}$ (for any fixed $\mathbb{F}_{q}$ ) we have

$$
u_{2}^{m} u_{1}^{n}=\psi_{m}(q) \psi_{n}(q) \sum_{\underline{\operatorname{dim}} N=(m, n)} u_{[N]} .
$$

By $\S 4.4$,

$$
u_{2}^{m} u_{1}^{n}=\psi_{m}(q) \psi_{n}(q) u_{\left[V_{\beta} \oplus \cdots \oplus V_{\beta}\right]}+\psi_{m}(q) \psi_{n}(q) \sum u_{[P]} R_{l \delta} u_{[I]},
$$

where $P$ is preprojective, $I$ is preinjective, $\underline{\operatorname{dim}} P+l \delta+\underline{\operatorname{dim}} I=(m, n)$, and $\operatorname{dim} \mathcal{O}_{P\left(\mathbf{c}^{\prime}\right),(l) I\left(\mathbf{c}^{\prime}\right)}<\operatorname{dim} \mathcal{O}_{M(\mathbf{c}(\beta) \beta)}$. Although the number of terms in $R_{l \delta}=$ $\sum_{[M]} u_{[M]}$ in $\mathcal{H}_{q}$ depends on $q$, Lemma 5.8 shows that $R_{l \delta}$ has a generic form in $\mathcal{C}_{\mathcal{Z}}^{*}$ with each component in $\mathcal{H}_{q}$ being $R_{l \delta}$. Then in $\mathcal{C}_{\mathcal{Z}}^{*}$,

$$
\begin{aligned}
u_{2}^{(* m)} * u_{1}^{(* n)} & =\frac{v^{m(m-1) / 2} v^{n(n-1) / 2}}{[m] ![n] !} v^{-2 m n} u_{2}^{m} u_{1}^{n} \\
& =v^{m^{2}-m+n^{2}-n-2 m n} u_{\left[V_{\beta} \oplus \cdots \oplus V_{\beta}\right]}+v^{m^{2}-m+n^{2}-n-2 m n} \sum u_{[P]} R_{l \delta} u_{[I]} \\
& =\left\langle u_{\mathbf{c}(\beta) \beta}\right\rangle+\sum_{\substack{\mathbf{c}^{\prime} \in \mathbb{N}_{f}^{\Phi+} \\
\operatorname{dim} \mathcal{O}_{\mathbf{c}^{\prime}}<\operatorname{dim} \mathcal{O}_{\mathbf{c}(\beta) \beta}}} v^{\lambda\left(\mathbf{c}^{\prime}\right)} E^{\mathbf{c}^{\prime}} .
\end{aligned}
$$

Similarly, we have the following:

Lemma 9.3. Let $\mathbf{c}, \mathbf{c}^{\prime} \in \mathbb{N}_{f}^{\Phi^{+}}$be such that $M(\mathbf{c})=P(\mathbf{c})$ and $M\left(\mathbf{c}^{\prime}\right)=I\left(\mathbf{c}^{\prime}\right)$. In $\mathcal{C}_{\mathcal{Z}}^{*}$ we have

$$
\begin{aligned}
& E(\mathbf{c})=\langle P(\mathbf{c})\rangle+\sum_{\substack{\mathbf{c}^{\prime \prime} \in \mathbb{N}_{+}^{\Phi+} \\
\operatorname{dim} \mathcal{O}_{\mathbf{c}^{\prime \prime}}<\operatorname{dim} \mathcal{O}_{\mathbf{c}}}} g_{\mathbf{c}^{\prime \prime}}^{\mathbf{c}} E^{\mathbf{c}^{\prime \prime}} \\
& E\left(\mathbf{c}^{\prime}\right)=\left\langle I\left(\mathbf{c}^{\prime}\right)\right\rangle+\sum_{\substack{\mathbf{c}^{\prime \prime} \in \mathbb{N}_{f}^{\Phi+} \\
\operatorname{dim} \mathcal{O}_{\mathbf{c}^{\prime \prime}}<\operatorname{dim} \mathcal{O}_{\mathbf{c}^{\prime}}}} h_{\mathbf{c}^{\prime \prime}}^{\mathbf{c}^{\prime}} E^{\mathbf{c}^{\prime \prime}}, \quad \text { where } g_{\mathbf{c}^{\prime \prime}}^{\mathbf{c}}, h_{\mathbf{c}^{\prime \prime}}^{\mathbf{c}^{\prime}} \in \mathcal{Z} .
\end{aligned}
$$


Proof. Since Hall polynomials exist in the Kronecker quiver, the lemma follows from Lemma 9.2, Proposition 6.5, and Lemma 4.4.

Lemma 9.4. Let $n, m \geq 1$. Then

$$
E(n \delta) * E(m \delta)=E_{n \delta} * E_{m \delta}+\sum_{\substack{\mathbf{c} \in \mathbb{N}_{f}^{\Phi+} \\ \operatorname{dim} \mathcal{O}_{\mathbf{c}}<\operatorname{dim} \mathcal{N}_{(n+m) \delta}}} h(\mathbf{c}) E^{\mathbf{c}}, \quad \text { where } h(\mathbf{c}) \in \mathcal{Z} .
$$

Proof. By Lemma 5.11(iii), we have

$$
\begin{gathered}
E(n \delta)=E_{n \delta}+\sum_{P \neq 0, I \neq 0} v^{l\left(\langle P\rangle * E_{l \delta} *\langle I\rangle\right)}\langle P\rangle * E_{l \delta} *\langle I\rangle, \\
E(m \delta)=E_{m \delta}+\sum_{P \neq 0, I \neq 0} v^{l\left(\langle P\rangle * E_{l \delta} *\langle I\rangle\right)}\langle P\rangle * E_{l \delta} *\langle I\rangle,
\end{gathered}
$$

where $l\left(\langle P\rangle * E_{l \delta} *\langle I\rangle\right) \in \mathbb{Z}$. We then have

$$
E(n \delta) * E(m \delta)=E_{n \delta} * E_{m \delta}+\sum h(\mathbf{c}) E^{\mathbf{c}} .
$$

It is easy to see from the structure of the AR-quiver of the Kronecker quiver that there is a real root $\alpha$ so that $\mathbf{c}(\alpha) \neq 0$ for each $\mathbf{c}$. Thus $\operatorname{dim} \mathcal{O}_{\mathbf{c}}<\operatorname{dim} \mathcal{N}_{(n+m) \delta}$.

Remark. By Lemma 9.4 we can get

$E(\omega \delta)=E\left(\omega_{1} \delta\right) * \cdots * E\left(\omega_{m} \delta\right)=E_{\omega \delta}+\sum_{\operatorname{dim} \mathcal{O}_{\mathbf{c}}<\operatorname{dim} \mathcal{O}_{\omega \delta}} h(\mathbf{c}) E^{\mathbf{c}}, \quad$ where $h(\mathbf{c}) \in \mathcal{Z}$

Let $\varphi: \mathbb{N}_{f}^{\Phi^{+}} \rightarrow \mathbb{N}^{2}$ be defined by $\varphi(\mathbf{c})=\sum_{\alpha \in \Phi^{+}} \mathbf{c}(\alpha) \alpha$. Then for any $\mathbf{d} \in \mathbb{N}^{2}$, $\varphi^{-1}(\mathbf{d})$ is a finite set. We define a (geometric) order in $\varphi^{-1}(\mathbf{d})$ as follows: $\mathbf{c}^{\prime} \preceq \mathbf{c}$ if and only if either $\mathbf{c}^{\prime}=\mathbf{c}$ or $\mathbf{c}^{\prime} \neq \mathbf{c}$ but $\operatorname{dim} \mathcal{O}_{\mathbf{c}^{\prime}}<\operatorname{dim} \mathcal{O}_{\mathbf{c}}$.

From Lemma 2.3(vii) and the above lemmas, we may summarize our results of this subsection as follows, which resembles [L1, 7.8].

Proposition 9.5. For any $\mathbf{c} \in \mathbb{N}_{f}^{\Phi^{+}}$, we have

$$
E(\mathbf{c})=\sum_{\mathbf{c}^{\prime} \in \varphi^{-1}(\varphi(\mathbf{c}))} h_{\mathbf{c}^{\prime}}^{\mathbf{c}} E^{\mathbf{c}^{\prime}}
$$

where
(i) $h_{\mathbf{c}^{\prime}}^{\mathbf{c}} \in \mathcal{Z}$,
(ii) $h_{\mathbf{c}}^{\mathbf{c}}=1$,
(iii) if $h_{\mathbf{c}^{\prime}}^{\mathbf{c}} \neq 0$ then $\mathbf{c}^{\prime} \preceq \mathbf{c}$,
(iv) $\overline{E(\mathbf{c})}=E(\mathbf{c})$. 
In the same way as in $\S 8.4$, we define $e^{\mathbf{c}}=\langle P(\mathbf{c})\rangle * S_{w_{\mathbf{c}} \delta} *\langle I(\mathbf{c})\rangle$ and the partial order $\prec$ for $e^{\mathbf{c}}$ is the same as in $\S 8.4$. We then have the following:

Proposition 9.6. For any $\mathbf{c} \in \mathbb{N}_{f}^{\Phi^{+}}$, we have

$$
E(\mathbf{c})=\sum_{\mathbf{c}^{\prime} \in \varphi^{-1}(\varphi(\mathbf{c}))} g_{\mathbf{c}^{\prime}}^{\mathbf{c}} e^{\mathbf{c}^{\prime}}
$$

where

(i) $g_{\mathbf{c}^{\prime}}^{\mathbf{c}} \in \mathcal{Z}$,

(ii) $g_{\mathbf{c}}^{\mathbf{c}}=1$,

(iii) if $g_{\mathbf{c}^{\prime}}^{\mathbf{c}} \neq 0$ then $\mathbf{c}^{\prime} \preceq \mathbf{c}$,

(iv) $\overline{E(\mathbf{c})}=E(\mathbf{c})$.

For any $\mathbf{c}, \mathbf{c}^{\prime} \in \mathbb{N}_{f}^{\Phi^{+}}$we define $\omega_{\mathbf{c}^{\prime}}^{\mathbf{c}} \in \mathcal{Z}$ by

$$
\overline{e^{\mathbf{c}}}=\sum_{\mathbf{c}^{\prime} \in \mathbb{N}_{f}^{\Phi+}} \omega_{\mathbf{c}^{\prime}}^{\mathbf{c}} e^{\mathbf{c}^{\prime}} .
$$

The following proposition resembles [L1, Prop. 7.9].

Proposition 9.7. $\omega_{\mathbf{c}}^{\mathbf{c}}=1$, and if $\omega_{\mathbf{c}^{\prime}}^{\mathbf{c}} \neq 0$ and $\mathbf{c}^{\prime} \neq \mathbf{c}$ then $\mathbf{c}^{\prime} \prec \mathbf{c}$.

Proof. Since $\overline{E(\mathbf{c})}=E(\mathbf{c})$ and $\left\{E^{\mathbf{c}} \mid \mathbf{c} \in \mathbb{N}_{f}^{\Phi^{+}}\right\}$is a $\mathcal{Z}$-basis of $\mathcal{C}^{*}$, we have

$$
g_{\mathbf{c}^{\prime \prime}}^{\mathbf{c}}=\sum_{\mathbf{c}^{\prime}} \overline{g_{\mathbf{c}^{\prime}}^{\mathbf{c}}} \omega_{\mathbf{c}^{\prime \prime}}^{\mathbf{c}^{\prime}} \quad \text { for } \mathbf{c}, \mathbf{c}^{\prime \prime} \in \varphi^{-1}(\mathbf{d})
$$

By Proposition 9.5, the matrices $\left(h_{\mathbf{c}^{\prime \prime}}^{\mathbf{c}}\right)$ as well as $\left(\overline{h_{\mathbf{c}^{\prime}}^{\mathbf{c}}}\right)$, where the index set is $\varphi^{-1}(\mathbf{d})$, are triangular with 1 s on the diagonal. Hence, by the equation above, the matrix $\left(\omega_{\mathbf{c}^{\prime \prime}}^{\mathbf{c}}\right)$ has the same property.

Consider the bar involution $\overline{(~)}: \mathcal{C}^{*} \rightarrow \mathcal{C}^{*}$. For any $\mathbf{c} \in \mathbb{N}_{f}^{\Phi^{+}}$, the equality

$$
e^{\mathbf{c}}=\overline{\overline{e^{\mathbf{c}}}}=\overline{\sum_{\mathbf{c}^{\prime}} \omega_{\mathbf{c}^{\prime}}^{\mathbf{c}} e^{\mathbf{c}^{\prime}}}=\sum_{\mathbf{c}^{\prime}, \mathbf{c}^{\prime \prime}} \overline{\omega_{\mathbf{c}^{\prime}}^{\mathbf{c}}} \omega_{\mathbf{c}^{\prime \prime}}^{\mathbf{c}^{\prime}} e^{\mathbf{c}^{\prime \prime}}
$$

implies the orthogonal relation

$$
\sum_{\mathbf{c}^{\prime}} \overline{\omega_{\mathbf{c}^{\prime}}^{\mathbf{c}}} \omega_{\mathbf{c}^{\prime \prime}}^{\mathbf{c}^{\prime}}=\delta_{\mathbf{c c}^{\prime \prime}}
$$

Therefore one can solve uniquely the system of equations

$$
\zeta_{\mathbf{c}^{\prime}}^{\mathbf{c}}=\sum_{\mathbf{c}^{\prime} \preceq \mathbf{c}^{\prime \prime} \preceq \mathbf{c}} \omega_{\mathbf{c}^{\prime}}^{\mathbf{c}^{\prime \prime} \overline{\zeta_{\mathbf{c}^{\prime \prime}}^{\mathbf{c}}}}
$$


with unknowns $\zeta_{\mathbf{c}^{\prime}}^{\mathbf{c}} \in \mathbb{Z}\left[v^{-1}\right], \mathbf{c}^{\prime} \preceq \mathbf{c}$ and $\mathbf{c}^{\prime}, \mathbf{c} \in \varphi^{-1}(\mathbf{d})$, such that

$$
\zeta_{\mathbf{c}}^{\mathbf{c}}=1 \quad \text { and } \quad \zeta_{\mathbf{c}^{\prime}}^{\mathbf{c}} \in v^{-1} \mathbb{Z}\left[v^{-1}\right] \quad \text { for all } \mathbf{c}^{\prime} \prec \mathbf{c} .
$$

For any $\mathbf{d} \in \mathbb{N}^{2}$ and $\mathbf{c} \in \varphi^{-1}(\mathbf{d})$, we set

$$
\mathcal{E}^{\mathbf{c}}=\sum_{\mathbf{c}^{\prime} \in \varphi^{-1}(\mathbf{d})} \zeta_{\mathbf{c}^{\prime}}^{\mathbf{c}} e^{\mathbf{c}^{\prime}} \text { and } \mathbf{J}=\left\{\mathcal{E}^{\mathbf{c}} \mid \mathbf{c} \in \varphi^{-1}(\mathbf{d}), \mathbf{d} \in \mathbb{N}^{2}\right\} .
$$

Let

$$
\mathcal{L}=\operatorname{span}_{\mathbb{Z}\left[v^{-1}\right]}\left\{e^{\mathbf{c}} \mid \mathbf{c} \in \mathbb{N}_{f}^{\Phi^{+}}\right\} .
$$

We verify the following two properties of $\mathbf{J}$. The first is

$$
\overline{\mathcal{E}^{\mathbf{c}}}=\sum_{\mathbf{c}^{\prime}} \overline{\zeta_{\mathbf{c}^{\prime}}^{\mathbf{c}}} \overline{e^{\mathbf{c}^{\prime}}}=\sum_{\mathbf{c}^{\prime}} \overline{\zeta_{\mathbf{c}^{\prime}}^{\mathbf{c}}} \sum_{\mathbf{c}^{\prime \prime}} \omega_{c^{\prime \prime}}^{\mathbf{c}^{\prime}} e^{\mathbf{c}^{\prime \prime}}=\sum_{\mathbf{c}^{\prime \prime}}\left(\sum_{\mathbf{c}^{\prime}} \overline{\zeta_{\mathbf{c}^{\prime}}^{\mathbf{c}}} \omega_{c^{\prime \prime}}^{\mathbf{c}^{\prime}}\right) e^{\mathbf{c}^{\prime \prime}}=\sum_{\mathbf{c}^{\prime \prime}} \zeta_{\mathbf{c}^{\prime \prime}}^{\mathbf{c}} e^{\mathbf{c}^{\prime \prime}}=\mathcal{E}^{\mathbf{c}}
$$

So the elements $\mathcal{E}^{\mathbf{c}}$ are bar-invariant. The second property is obvious: $\mathbf{J}$ is a $\mathbb{Z}\left[v^{-1}\right]$ basis of the lattice $\mathcal{L}$. Therefore we have

Proposition 9.8. The set $\mathbf{J}$ is a basis of $\mathcal{C}_{\mathcal{Z}}^{*}$ which satisfies

(i) $\overline{\mathcal{E}^{\mathbf{c}}}=\mathcal{E}^{\mathrm{c}}$,

(ii) $\left(\mathcal{E}^{\mathbf{c}}, \mathcal{E}^{\mathbf{c}^{\prime}}\right) \equiv \delta_{\mathbf{c}, \mathbf{c}^{\prime}}\left(\bmod v^{-1} \mathbb{Z}\left[\left[v^{-1}\right]\right] \cap \mathbb{Q}(v)\right)$,

(iii) $\pi\left(\mathcal{E}^{\mathbf{c}}\right)=\pi\left(E^{\mathbf{c}}\right)$,

for any $\mathcal{E}^{\mathbf{c}} \in \mathcal{L}$, where $\pi$ is the canonical projection $\mathcal{L} \rightarrow \mathcal{L} / v^{-1} \mathcal{L}$.

By the total positivity of the canonical basis, we find that $\mathbf{J}$ is just the canonical basis in the sense of Lusztig.

\section{Acknowledgements}

This research was supported in part by NSF grant 10771112 by NSCF grant 10931006, by the 973 Project of the Ministry of Science and Technology of China, by NSF grant DMS-0200673 and by STCSM(09XD1402500).

The main results of this article were reported by J. Xiao at the Fields Institute, Toronto, Canada (Workshop and Conference on Infinite Dimensional Lie Theory and its Application, July 17-25, 2003), at the Algebra seminar of the University of Sydney, Australia, October 10, 2003 and at Snowbird, Utah, USA (AMSIMS-SIAM Summer Research Conferences, Representations of Algebraic Groups, Quantum Groups, and Lie Algebras, July 11-15, 2004).

The authors are grateful to O. Schiffmann, B. Deng and J. Du for calling their attention to the preprint $[\mathrm{H}]$. We thank H. Nakajima for his encouragement 
to answer his question in $[\mathrm{N}]$, in particular, for his suggestion to use Schur functions to modify $E_{n \delta}$ 's. We thank F. Xu for reading through the first version of this paper. Finally we thank the referee for detailed comments on earlier versions of the paper suggesting the current re-organization of the sections to make the presentation more readable and for the patience of reading through the paper several times.

\section{References}

[BK] P. Baumann and C. Kassel, The Hall algebra of the category of coherent sheaves on the projective line, J. Reine Angew. Math. 533 (2001), 207-233. Zbl 0967.18005 MR 1823869

[Be] J. Beck, Braid group action and quantum affine algebras, Comm. Math. Phys. 165 (1994), 555-568. Zbl 0807.17013 MR 1301623

[BCP] J. Beck, V. Chari and A. Pressley, An algebraic characterization of the affine canonical basis, Duke Math. J. 99 (1999), 455-487. Zbl 0964.17013 MR 1712630

[BN] J. Beck and H. Nakajima, Crystal bases and two sided cells of quantum affine algebras, Duke Math. J. 123 (2004), 335-402. Zbl 1062.17006 MR 2066942

[BGP] I. N. Bernstein, I. M. Gelfand and V. A. Ponomarev, Coxeter functors and Gabriel theorem, Uspekhi Mat. Nauk 28 (1973), no. 2, 19-33 (in Russian); English transl.: Russian Math. Surveys 28 (1973), no. 2, 17-32. Zbl 0279.08001 MR 0393065

[C] X. Chen, Root vectors of the composition algebra of the Kronecker algebra, Algebra Discrete Math. 2004, no. 1, 37-56. Zbl 1067.16019 MR 2051380

[CX] X. Chen and J. Xiao, Exceptional sequences in Hall algebras and quantum groups, Compos. Math. 117 (1999), 161-187. Zbl 0993.16008 MR 1695853

[CB] W. Crawley-Boevey, Lectures on representations of quivers, Math. Inst., Oxford Univ., 1992.

[Da] I. Damiani, A basis of type Poincaré-Birkhoff-Witt for the quantum algebra of $\widehat{s l}_{2}$, J. Algebra 161(1993), 291-310. Zbl 0803.17003 MR 1247357

[DDX] B. Deng, J. Du and J. Xiao, Generic extensions and canonical bases for cyclic quivers, Canad. J. Math. 59 (2007), 1260-1283. Zbl 1203.17007 MR 2363066

[DR] V. Dlab and C. M. Ringel, Indecomposable representations of graphs and algebras, Mem. Amer. Math. Soc. 6 (1976), no. 173, 57 pp. Zbl 0332.16015 MR 0447344

[Dr] V. G. Drinfeld, A new realization of Yangians and of quantum affine algebras, Soviet Math. Dokl. 36 (1988), 212-216. MR 0914215

[DL] M. Dyer and G. Lusztig, Appendix to "Quantum groups at roots of 1", Geom. Dedicata 35 (1990), 89-113.

[FMV] I. Frenkel, A. Malkin and M. Vybornov, Affine Lie algebras and tame quivers, Selecta Math. (N.S.) 7 (2001), 1-56. Zbl 1002.16012 MR 1856552

[G] J. A. Green, Hall algebras, hereditary algebras and quantum groups, Invent. Math. 120 (1995), 361-377. Zbl 0836.16021 MR 1329046

[GL] I. Grojnowski and G. Lusztig, A comparison of bases of quantized enveloping algebras, in Linear algebraic groups and their representations, Contemp. Math. 153, Amer. Math. Soc., 1993, 11-19. Zbl 1009.17502 MR 1247495

[H] A. Hubery, The composition algebra of an affine quiver, arXiv:math.RT/0403206. 
[Ka] V. Kac, Infinite dimensional Lie algebras, 3rd ed., Cambridge Univ. Press, Cambridge, 1990. Zbl 0716.17022 MR 1104219

[K1] M. Kashiwara, Crystalizing the $q$-analogue of universal enveloping algebras, Comm. Math. Phys. 133 (1990), 249-260. Zbl 0724.17009 MR 1090425

[K2] On crystal bases of the $q$-analogue of universal enveloping algebras, Duke Math. J. 63 (1991), 465-516. Zbl 0739.17005 MR 1115118

[Li] Y. Li, Notes on affine canonical and monomial bases, arXiv:math.RT/0610449.

[LL] Y. Li and Z. Lin, Ar-quiver approach to affine canonical basis elements, J. Algebra 318 (2007), 562-588. Zbl 1181.17006 MR 2371959

[Lin] Z. Lin, Lusztig's geometric approach to Hall algebras, in Representations of finite dimensional algebras and related topics in Lie theory and geometry, Fields Inst. Comm. 40, Amer. Math. Soc., Providence, RI, 2004, 349-364. Zbl 1046.17007 MR 2057403

[L1] G. Lusztig, Canonical bases arising from quantized enveloping algebras, J. Amer. Math. Soc. 3 (1990), 447-498. Zbl 0703.17008 MR 1035415

[L2] Luivers, perverse sheaves, and the quantized enveloping algebras, J. Amer. Math. Soc. 4 (1991), 365-421. Z Zbl 0738.17011 MR 1088333

[L3] _ Affine quivers and canonical bases, Inst. Hautes Études Sci. Publ. Math. 76 (1992), 111-163. Zbl 0776.17013 MR 1215594

[L4] Canonical bases and Hall algebras, in Representation theories and algebraic geometry, A. Broer and A. Daigneault (eds.), Kluwer, 1998, 365-399. Zbl 0934.17010 MR 1653038

[L5] _ Introduction to quantum groups, Birkhäuser, Boston, 1993. Z Zbl 0788.17010 MR 1227098

[L6] Left cells in Well groups, in Lie group representations, I, Lecture Notes in Math. 1024, Springer, 1983, 99-111.

[L7] , Quantum groups at roots of 1, Geom. Dedicata 35 (1990), 89-113. Zbl 0714.17013 MR 1066560

[M] I. G. Macdonald, Symmetric functions and Hall polynomials, Oxford Math. Monogr., Clarendon Press, Oxford, 1995. Zbl 0824.05059 MR 1354144

[N] H. Nakajima, Crystal, canonical and PBW bases of quantum affine algebras, in Algebraic groups and homogeneous spaces, V. B. Mehta (ed.), Narosa Publ. House, 2007, 389-421. Zbl 1194.17009 MR 2348912

[Re] M. Reineke, The monoid of families of quiver representations, Proc. London Math. Soc. 84 (2002), 663-685. Zbl 1064.16015 MR 1888427

[R1] C. M. Ringel, Hall algebras and quantum groups, Invent. Math. 101 (1990), 583-592. Zbl 0735.16009 MR 1062796

[R2] , The composition algebra of a cyclic quiver, Proc. London Math. Soc. 66 (1993), 507-537. Zbl 0797.16014 MR 1207546

[R3] , PBW-bases of quantum groups, J. Reine Angew. Math. 470 (1996), 51-88. Zbl 0840.17010

[R4] The Hall algebra approach to quantum groups, Aportaciones Mat. Comun. 15 (1995), 85-114. MR 1360930

[R5] Hall algebras, in Topics in algebra, Part 1, Banach Center Publ. 26, PWN Polish Sci. Publ., 1990, 433-447. Zbl 0778.16004 MR 1171248

[R6] - Tame algebras and integral quadratic forms, Lecture Notes in Math. 1099, Springer, Berlin, 1984. Zbl 0546.16013 MR 0774589 
[R7] Green's theorem on Hall algebras, in Representation theory of algebras and related topics, CMS Conf. Proc. 19, Amer. Math. Soc., Providence, RI, 1996, 185-245. Zbl 0860.16026 MR 1388564

[R8] The preprojective algebra of a quiver, in Algebras and modules, II (Geiranger, 1996), CMS Conf. Proc. 24, Amer. Math. Soc., Providence, RI, 1998, 467-480. Zbl 0928.16012 MR 1648647

[S] O. Schiffmann, The Hall algebra of a cyclic quiver and canonical bases of Fock spaces, Int. Math. Res. Notices 2000, 413-440. Zbl 0966.16005 MR 1753691

[SV] B. Sevenhant and M. Van den Bergh, A relation between a conjecture of Kac and the structure of the Hall algebra, J. Pure Appl. Algebra 160 (2001), 319-332. Zbl 1025.17006 MR 1836006

[VV] M. Varagnolo and E. Vasserot, On the decomposition matrices of the quantized Schur algebra, Duke Math. J. 100 (1999), 267-297. Zbl 0962.17006 MR 1722955

[XY] J. Xiao and S. Yang, BGP-reflection functors and Lusztig's symmetries: a Ringel-Hall algebra approach to quantum groups, J. Algebra 241 (2001), 204-246. Z Zbl 0999.17026 MR 1838851

[Z1] P. Zhang, Composition algebras of affine type, J. Algebra 206 (1998), 505-540. Zbl 0973.17018 MR 1637072

[Z2] , PBW-bases of the composition algebra of the Kronecker algebra, J. Reine Angew. Math. 527 (2000), 97-116. Zbl 0958.16015 MR 1794019 